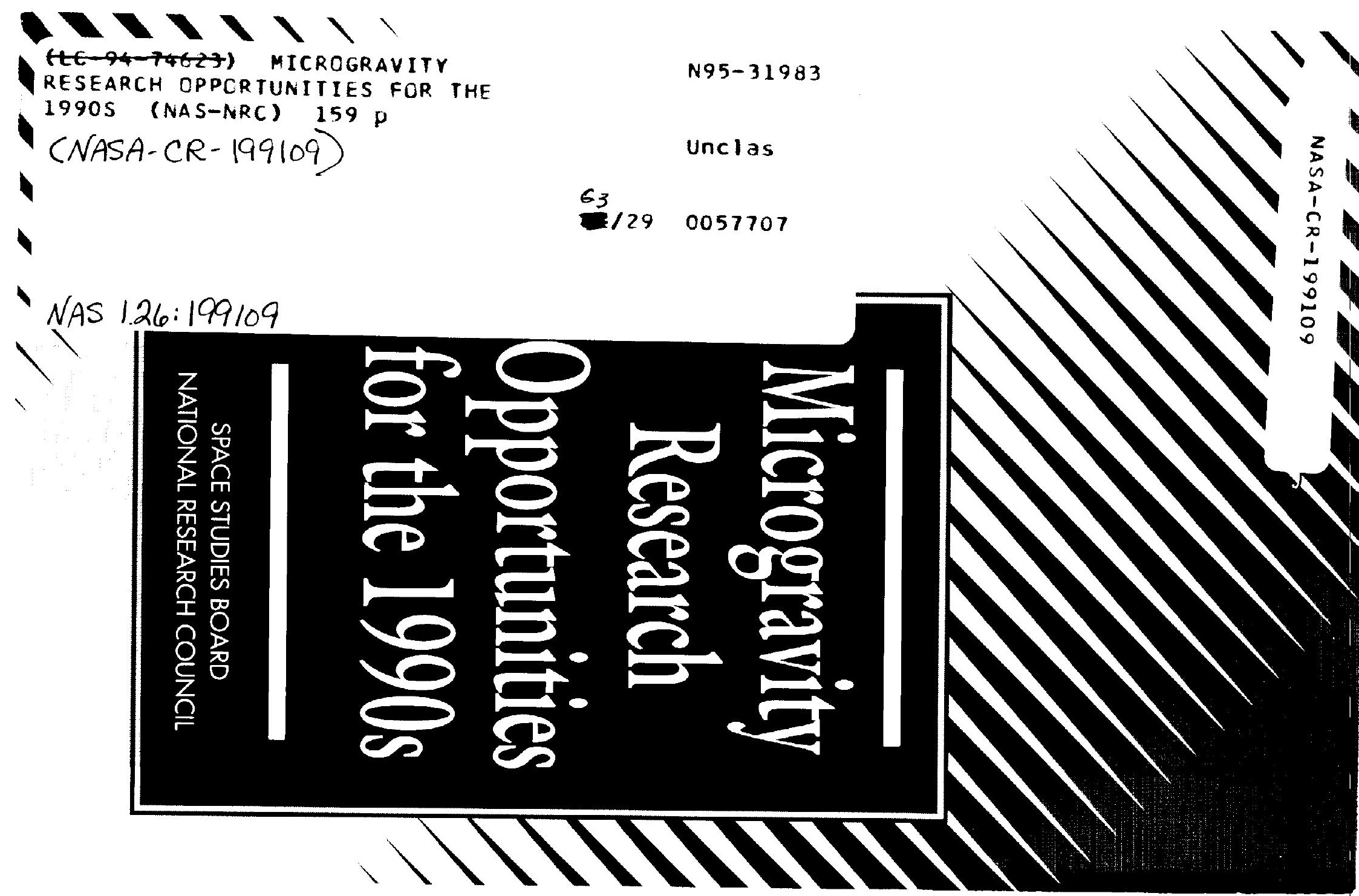


$\smile \smile$ 


\title{
Microgravity Research Opportunities for the 1990s
}

\author{
Committee on Microgravity Research \\ Space Studies Board \\ Commission on Physical Sciences, Mathematics, and Applications \\ National Research Council
}

Washington, D.C.

1995 
NOTICE: The project that is the subject of this report was approved by the Governing Board of the National Research Council, whose members are drawn from the councils of the National Academy of Sciences, the National Academy of Engineering, and the Institute of Medicine. The members of the committee responsible for the report were chosen for their special competences and with regard for appropriate balance.

This report has been reviewed by a group other than the authors according to procedures approved by a Report Review Committee consisting of members of the National Academy of Sciences, the National Academy of Engineering, and the Institute of Medicine.

The National Academy of Sciences is a private, nonprofit, self-perpetuating society of distinguished scholars engaged in scientific and engineering research, dedicated to the furtherance of science and technology and to their use for the general welfare. Upon the authority of the charter granted to it by the Congress in 1863, the Academy has a mandate that requires it to advise the federal government on scientific and technical matters. Dr. Bruce Alberts is president of the National Academy of Sciences.

The National Academy of Engineering was established in 1964, under the charter of the $\mathrm{Na}$ tional Academy of Sciences, as a parallel organization of outstanding engineers. It is autonomous in its administration and in the selection of its members, sharing with the National Academy of Sciences the responsibility for advising the federal government. The National Academy of Engineering also sponsors engineering programs aimed at meeting national needs, encourages education and research, and recognizes the superior achievements of engineers. Dr. Robert M. White is president of the National Academy of Engineering.

The Institute of Medicine was established in 1970 by the National Academy of Sciences to secure the services of eminent members of appropriate professions in the examination of policy matters pertaining to the health of the public. The Institute acts under the responsibility given to the National Academy of Sciences by its congressional charter to be an adviser to the federal government and, upon its own initiative, to identify issues of medical care, research, and education. Dr. Kenneth I. Shine is president of the Institute of Medicine.

The National Research Council was organized by the National Academy of Sciences in 1916 to associate the broad community of science and technology with the Academy's purposes of furthering knowledge and advising the federal government. Functioning in accordance with general policies determined by the Academy, the Council has become the principal operating agency of both the National Academy of Sciences and the National Academy of Engineering in providing services to the government, the public, and the scientific and engineering communities. The Council is administered jointly by both Academies and the Institute of Medicine. Dr. Bruce Alberts and Dr. Robert M. White are chairman and vice chairman, respectively, of the National Research Council.

Support for this project was provided by Contract NASW 4627 between the National Academy of Sciences and the National Aeronautics and Space Administration.

Library of Congress Catalog Card Number: $94-74623$

Copyright 1995 by the National Academy of Sciences. All rights reserved.

Copies of this report are available from:

Space Studies Board

National Research Council

2101 Constitution Avenue, N.W.

Washington, D.C. 20418

Printed in the United States of America 
COMMITTEE ON MICROGRAVITY RESEARCH

WILLIAM A. SIRIGNANO,* University of California, Irvine, Chair ROSALIA N. ANDREWS, * University of Alabama, Birmingham HOWARD M. EINSPAHR, Bristol Myers Squibb Company MARTIN E. GLICKSMAN, Rensselaer Polytechnic Institute FRANKLIN D. LEMKEY,* United Technologies Research Center RONALD E. LOEHMAN, Sandia Laboratories ALEXANDER McPHERSON,* University of California, Riverside SIMON OSTRACH,* Case Western Reserve University MORTON B. PANISH, AT\&T Bell Laboratories (retired) JOHN D. REPPY, Cornell University WARREN C. STRAHLE,* Georgia Institute of Technology (deceased) JULIA WEERTMAN, ${ }^{*}$ Northwestern University

RICHARD C. HART, Senior Program Officer SANDRA J. GRAHAM, Senior Program Officer CARMELA J. CHAMBERLAIN, Senior Program Assistant VICTORIA P. FRIEDENSEN, Senior Program Assistant

* Former member 


\section{SPACE STUDIES BOARD}

CLAUDE R. CANIZARES, Massachusetts Institute of Technology, Chair LOUIS J. LANZEROTTI,* AT\&T Bell Laboratories, Chair

JOHN A. ARMSTRONG, IBM Corporation (retired)

JOSEPH A. BURNS, Cornell University

JOHN J. DONEGAN, U.S. Navy (retired)

JOHN A. DUTTON,* Pennsylvania State University

ANTHONY W. ENGLAND, University of Michigan

JAMES P. FERRIS, * Rensselaer Polytechnic Institute

DANIEL J. FINK, D.J. Fink and Associates, Inc.

HERBERT FRIEDMAN, ${ }^{*}$ Naval Research Laboratory

MARTIN E. GLICKSMAN, Rensselaer Polytechnic Institute

HAROLD J. GUY, University of California, San Diego

NOEL W. HINNERS, Martin Marietta Astronautics Company

ROBERT A. LAUDISE, AT\&T Bell Laboratories

RICHARD S. LINDZEN,* Massachusetts Institute of Technology

JANET G. LUHMANN, University of California, Berkeley

JOHN H. McELROY, University of Texas, Arlington

WILLIAM J. MERRELL, JR., ${ }^{*}$ Texas A\&M University

NORMAN F. NESS, * University of Delaware

MARCIA NEUGEBAUER, Jet Propulsion Laboratory

MARY JANE OSBORN, University of Connecticut Health Center

SIMON OSTRACH. Case Western Reserve University

JEREMIAH P. OSTRIKER, Princeton University

CARLÉ M. PIETERS, Brown University

JUDITH PIPHER, University of Rochester

MARCIA J. RIEKE, University of Arizona

ROLAND W. SCHMITT, Clifton, New Jersey

WILLIAM A. SIRIGNANO, * University of California, Irvine

JOHN W. TOWNSEND, * NASA (retired)

FRED W. TUREK,* Northwestern University

ARTHUR B.C. WALKER, JR., Stanford University

MARC S. ALLEN, Director

* Former member 


\section{COMMISSION ON PHYSICAL SCIENCES, MATHEMATICS, AND APPLICATIONS}

RICHARD N. ZARE, Stanford University, Chair

RICHARD S. NICHOLSON, American Association for the Advancement of Science, Vice Chair

STEPHEN L. ADLER, Institute for Advanced Study SYLVIA T. CEYER, Massachusetts Institute of Technology SUSAN L. GRAHAM, University of California, Berkeley ROBERT J. HERMANN, United Technologies Corporation RHONDA J. HUGHES, Bryn Mawr College SHIRLEY A. JACKSON, Rutgers University KENNETH I. KELLERMANN, National Radio Astronomy Observatory HANS MARK, University of Texas, Austin THOMAS A. PRINCE, California Institute of Technology JEROME SACKS, National Institute of Statistical Sciences L.E. SCRIVEN, University of Minnesota A. RICHARD SEEBASS III, University of Colorado LEON T. SILVER, California Institute of Technology CHARLES P. SLICHTER, University of Illinois ALVIN W. TRIVELPIECE, Oak Ridge National Laboratory SHMUEL WINOGRAD, IBM T.J. Watson Research Center CHARLES A. ZRAKET, MITRE Corporation (retired)

NORMAN METZGER, Executive Director 



\section{Preface}

For many years, the Space Studies Board (SSB) has provided scientific advice to the National Aeronautics and Space Administration (NASA) in discipline-oriented strategy reports intended to assist NASA in developing the best possible scientific research programs for the future. In 1989, the SSB created the Committee on Microgravity Research (CMGR) to investigate the maturity and readiness of the field for the development of a long-range, comprehensive research strategy. The report of that committee, Toward a Microgravity Research Strategy (National Academy Press, Washington, D.C., 1992), concluded that "the field as a whole would benefit from the formulation of a long-range research strategy and that such a strategy should be developed as soon as possible" (p. 1).

The CMGR was then made a standing committee of the SSB and was charged with developing that strategy. After careful consideration of the current status and content of the microgravity program at NASA, the committee found it necessary to modify this charge somewhat. Due to the diversity and breadth of this field, the committee has chosen to place the emphasis of this report on priorities and recommendations. The scientific disciplines contained within the microgravity program, and which are covered in this report, include fluid mechanics and transport phenomena, combustion, biological sciences and biotechnology, materials science, and microgravity physics. This represents an enormously broad spectrum of science, and a determination of the relative amount of support that is justified for individual disciplines and subdisciplines is difficult to make without a set of overriding criteria (such as the value to industry) to use in the evaluation process. In the absence of such criteria, the formulation of a detailed program strategy was not possible. Therefore, the committee believed that providing general program guidance in the form of priorities and recommen-

$v i i$

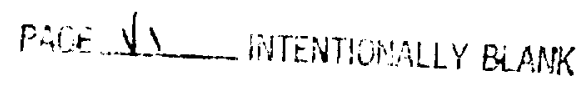

\section{AKECEDING PAGE BLANK NOT FLMED}


dations for research was a more useful and appropriate response to the given charge. The following report represents the result of that effort.

Among the previous National Research Council reports in this field, the following are particularly relevant.

- Materials Processing in Space (National Academy of Sciences, Washington, D.C., 1978; the "STAMPS" report) attempted to provide guidance for NASA's program by assessing the scientific and technological underpinnings of the materials processing in space program and providing a clear understanding of the benefits, if any, to be expected from exploitation of the characteristics of the space environment for processing materials. As an early examination of NASA's low-gravity experiment program (pre-shuttle), the report addressed fundamental scientific and administrative questions about the directions that program should follow. One such issue was the production of materials in space for commercial use, which the report concluded was not feasible. The scientific recommendations detailed the specific research areas most likely to benefit from a low-gravity environment, and the administrative recommendations included a call for a rigorous peer review process and an extensive ground research program. In fact, the current NASA program closely mirrors the report's recommendations. Though recent flight experiments are considerably more sophisticated than those examined by the STAMPS committee, the continuing scarcity of available flight results requires that assessments of the utility of many areas of microgravity research be based on their potential rather than on past performance.

- Space Science in the Twenty-First Century: Imperatives for the Decades 1995 to 2015. Fundamental Physics and Chemistry (National Academy Press, Washington, D.C., 1988) attempted to identify opportunities for future research efforts in relativistic gravitation and microgravity science (but not including applied research or industrial and manufacturing processes). NASA requested this study in 1984, in part because it expected the space station to become available around 1995 and wanted to be prepared with a viable space science strategy that had been implemented on missions prior to space station operations. At the time the report was written, NASA's microgravity research was concentrated under the Physics and Chemistry Experiments in Space (PACE) program. Only about 15 investigations were funded under $P A C E$, in comparison with more than 200 investigations in the 1993 microgravity program at NASA. The report reflected the relatively small scale of the early program by primarily addressing specific research questions as opposed to the broad disciplines into which microgravity research is now divided.

A number of people who assisted the committee during its preparation of this report deserve special thanks for their contributions: Robert Rhome, Roger Crouch, Gary Martin, and Brad Carpenter of NASA Headquarters; John Givens and George Sarver of NASA Ames Research Center; Jack Salzman of Lewis Research Center: Dudley Saville of Princeton University; and Gregory Dobbs of United Technologies Research Center. 


\section{Contents}

EXECUTIVE SUMMARY

Introduction, 1

Scope of the Recommendations, 3

Recommendations for the Science Program, 5

General Science Recommendations for Microgravity

Research, 14

Flight Opportunities and Challenges, 16

Administrative Recommendations, 18

References, 19

\section{PART I-OVERVIEW}

1 Introduction

The Microgravity Environment, 24

Perceptions and Realities of Microgravity Research, 25

References, 27

2 Conclusions and Recommendations

Introduction, 28

Areas Recommended for Emphasis in Microgravity Research, 30

General Science Recommendations for Microgravity Research, 39

Administrative Recommendations, 40

Reference, 42 


\section{PART II-SCIENTIFIC ISSUES}

3 Fluid Mechanics and Transport Phenomena

Introduction, 45

Status, 46

Research Areas, 48

Summary, 51

References, 51

4 Combustion

Introduction and Overall Goals, 53

Problems Unique to Combustion, 54

Status of Gravitational-Based Current Knowledge, 56

Special Instrumentation and Facilities Requirements, 58

Recommendations and Conclusions, 59

References, 60

5 Biological Sciences and Biotechnology

Introduction, 62

Justification for Microgravity Research, 62

Fundamental Research Areas, 65

Experimental Requirements, 72

Recommendations and Conclusions, 73

References, 74

6 Materials Science and Processing

Metals and Alloys, 76

Background and Introduction, 76

Fundamental Research Areas-Metals and Alloys, 77

Future Scientific Directions-Metals and Alloys, 86

Recommendations and Conclusions, 87

POLymers, 88

Background, 88

Early Studies and Their Current Relevance, 89

Recommended Studies, 91

Recommendations and Conclusions, 92

Growth of Inorganic Single Crystals, 93

Introduction and Goals, 93

Summary of Terrestrial Growth of Inorganic Single Crystals, 94

The Influence of Gravity, 95

Space- and Ground-Based Studies, 96

Recommendations and Conclusions, 99

Growth of Epitaxial Layers on Single-Crystal Substrates, 100

Epitaxial Growth Methods, 100

Recommendations and Conclusions, 101 
Ceramics and Glasses, 102

Introduction, 102

Current Issues in Ceramics Research, 102

Microgravity as a Variable in Ceramics Synthesis and Processing, 103

Ceramic and Glass Processing in Microgravity, 106

Basic Science, 107

Recommendations and Conclusions, 107

REFERENCES, 108

7 Microgravity Physics

Introduction, 111

The Current Microgravity Physics Program, 112

Future Experiments Under Microgravity Conditions, 115

Recommendations and Conclusions, 116

References, 117

\section{PART III-PROGRAMMATIC ISSUES}

8 Flight Opportunities and Challenges

Introduction, 121

Opportunities: Microgravity Research Facilities, 123

Challenges to Microgravity Research Experimentation, 129

\section{APPENDIXES}

A Glossary 137

B Biographies of Committee Members 146 

This report is dedicated to the memory of

Professor Warken C. Strahle (1937-1994),

a distinguished scholar and researcher who served as a vital contributor to this report. 



\section{Executive Summary}

\section{INTRODUCTION}

Microgravity research is concerned with the effects of reduced gravitational forces on physical, chemical, and biological phenomena. The scientific disciplines affected by gravity include fundamental physics, fluid mechanics and transport phenomena, materials science, biological sciences and biotechnology, and combustion. It is especially noteworthy that these disciplines are laboratory sciences that inherently use controlled, model experiments. Many experiments require constant attention and frequent intervention by the experimenter, which distinguishes microgravity research from the observational space sciences. $\mathrm{Mi}$ crogravity research also spans both fundamental and applied sciences.

Reduced-gravity experimental environments are provided by NASA through drop towers, aircraft in parabolic trajectories, sounding rockets, and Earth-orbiting laboratories. Some of these environments have crews and allow extended periods of time for experimentation and for demonstrating the reproducibility of results. Some of the experimental platforms allow a reduction of the gravity level to $10^{-6}$ times that of Earth gravity.

In a reduced-gravity environment, the decreases in rates of sedimentation, hydrostatic pressure, and buoyancy-driven flows cause other physical effects to become more important and more readily observable and measurable. The acceleration due to gravity can then be treated as an important and interesting experimental parameter. The exploration of this parameter, through experiments at normal Earth gravity and at reduced gravity, may provide a better understanding of certain physical processes, as well as lead to the identification of new phenomena. 
The NASA microgravity research program has been widely misperceived as simply a materials processing program with the goal of providing better products for use on Earth. However, the prospects of commercial manufacture in space are limited in the foreseeable future. The justification for the microgravity research program must continue to be the promise of advances in areas of fundamental and applied science. It should be recognized, on the other hand, that manned exploration of space will require increased understanding of transport phenomena. materials processing. and performance in microgravity.

There have been a number of previous National Research Council (NRC) reports in this field, of which the following are particularly relevant:

- Materials Processing in Space' (the "STAMPS" report, 1978) attempted to provide guidance for the future course of NASA's program. It assessed the scientific and technological underpinnings of the materials processing in space program and provided a clear understanding of the potential for exploitation of the space environment for processing materials.

- Space Sciche in the Twenty-First Centwry: Imperatives for the Decades 1995 to 2015. Fundamental Physics and Chemistry-2 (1988) allempted to identify opportunities for future research efforts in relativistic gravitation and microgravity science (but not including applied research or industrial/manufacturing processes).

- More recently, the report Toward a Microgravity Research Strategy.3 (1992) began to lay a foundation for a more mature research program, and the current report is a continuation of that effort.

The research areas discussed in this report are subsets of much broader research disciplines. Only a small fraction of the total activities in each discipline occurs within the microgravity program, and no attempt has been made here to evaluate fully or to prioritize all of the research in a discipline. Only the microgravity component of each discipline is addressed in detail. Furthermore, the cost-benefit of a microgravity program has not been compared to the costbenefit of experiments on different subjects in the terrestrial environment. Ex periments that can be performed adequately under terrestrial conditions, however, are not given a priority for spaceflight.

It should be understood at the outset that in evaluating the costs of doing research on such expensive vehicles as a space station, vehicle expenses are specifically not included. Microgravity research has never been the sole motivation for the space shuttle or other major space missions. These research opportunities have usually been secondary to exploratory, technological, engineering, political, educational, inspirational, and other motives for spaceflight, and until the advent of spacelab, microgravity research has been added on spaceflights launched for other purposes. Thus, in evaluating priorities, the research opportunities are taken into account but not the costs.

To date, only a limited number of microgravity experiments have been con- 
ducted in space with completed analyses and reports of results. The total experience of U.S.. Canadian, Japanese, and Western European scientists is less than 1000 hours for experiments in orbit. Because of the limited results available, the strategic recommendations in this report cannot be highly detailed or exclusive. A number of subjects require further exploratory investigation before detailed objectives can be defined. For the same reasons, it is currently not generally possible to set specific priorities across discipline lines. Assuming experiments are scientifically sound, prioritization across disciplines is largely unnecessary because a wide range of microgravity experiments can be accommodated on shuttle flights without retarding progress in any one discipline. It maly even be a mistake to attempt such prioritization since, in many cases, fundamental flight experiments are needed before prioritization can be attempted. Some areas. however, can be identified as more promising than others.

This report does not address the NASA commercial program or international programs in microgravity research. These topics will be addressed in future studies of this committee.

Finally, although the value and need for human intervention capabilities and Iong-duration flights are noted repeatedly in this report, nothing herein should necessarily be interpreted as advocating or opposing any specific NASA spacecraft or space station design initiative.

\section{SCOPE OF THE RECOMMENDATIONS}

The conclusions and recommendations presented in this report fall into five categories: (1) overall goals for the microgravity research program; (2) general priorities among the major scientific disciplines affected by gravity; (3) identification of the more promising experimental challenges and opportunities within each discipline; (4) general scientific recommendations that apply to all microgravity-related disciplines; and (5) recommendations concerning administrative policies and procedures that are essential to the conduct of excellent laboratory science.

The overall goal of the research program should be to advance science and technology in each of the component disciplines. Microgravity research should be aimed at making significant impacts in each discipline emphasized. The purpose of this report is to recommend means to accomplish that goal.

The essential features of the recommendations are to emphasize microgravity research for its general scientific and technological value, as well as its role in advancing technology for the exploration of space; to deemphasize the research value of manufacturing in space with the intent of returning products to Earth; to modify NASA 's infrastructure, policy, and procedures so as to facilitate laboratory science in space; to establish priorities for microgravity experimentation in scientific disciplines and subdisciplines in accordance with the relative opportunities for scientific and technological impact; and to recognize fluid mechanics 
and transport phenomena as a central theme throughout microgravity research. The recommendations detailed in this report are based on certain findings that resulted from this study. The findings are as follows:

- Science can be advanced by the study of certain mechanisms that are masked or dominated by gravitational effects at Earth gravity conditions. Examples include surface tension gradient-driven flows, capillary effects, multiphase flows, diffusive transport processes, and colloidal phenomena. Under terrestrial gravity conditions, these phenomena can often be dominated by effects such as buoyancy and sedimentation.

- There is a scientific need to understand better the role of gravity in many physical, chemical, and biological systems. To understand the relative importance of certain gravitational effects compared to nongravitational effects under terrestrial conditions, it is helpful to study a phenomenon at more than one gravitational level. Thus, gravitational force can become a controlled experimental parameter. For example, the role of buoyancy versus the role of surface tension gradients in driving a fluid flow could be examined at different gravitational levels.

- A significant portion of microgravity research programs should be driven by the technological needs of the overall space program. Examples of important engineering issues include the ignition, propagation, and extinction of spacecraft fires; the fluid dynamics and transport associated with the handling, storage, and use in space of water, waste, foods, fuels, air environments, and contaminants; the handling, joining, and reshaping of materials in space; and the dynamics and chemistry of mining or refining resources in extraterrestrial environments.

- Microgravity research primarily involves laboratory science with controlled, model experiments that inherently require attention and intervention by the experimenter. Such experiments also require the opportunity to demonstrate reproducibility.

- The potential for manufacturing in space in order to return economically competitive products to Earth is very small. Research on materials processing in microgravity, however, could prove to be important for materials science and materials processing technology on Earth.

- Fluid mechanics and transport phenomena represent both a distinct discipline and a scientific theme that impacts nearly all microgravity research experiments.

- The ground-based research program is critically important for the preparation and definition of the flight program.

The need for an extended-duration orbiting platform has been identified as critical in many microgravity research experiments because of the time required for experimentation, the wide parametric ranges, and the need to demonstrate the reproducibility of results. In developing this report, it has been assumed that a space station will be available within a decade. The recommendations have value and should be implemented, however, even if a space station does not 
materialize and the microgravity research program continues only with the current facilities.

Although this report does not set out a complete strategy for microgravity research, it presents some of the important elements of such a strategy, including:

- A summary of the current state of knowledge of microgravity science;

- A discussion of some of the fundamental questions to be answered;

- A presentation of the goals in this field;

- The science objectives within each discipline;

- An evaluation of the potential for microgravity research to provide advances within each discipline;

- The experimental requirements for achieving the science objectives of each discipline;

- A description of the other resources required for a successful microgravity science program; and

- A limited prioritization of research topics within each discipline.

The two aspects of a strategy for microgravity research that are not presented in this report are (1) a prioritization of microgravity research objectives across disciplines and (2) a cost-benefit analysis of anticipated microgravity results. Experience has shown how difficult it is to set research priorities, even within a single homogeneous science discipline. Reaching agreement on priorities for microgravity research relative to all other science research was judged so unlikely that it has not been attempted in this report. The other practical reason for not setting stricter priorities stems from the nature of shuttle flights. Frequently, payloads are assigned to a flight because their requirements for space, power, and so on, fit what is available, and scientific priority is less important. No costbenefit analysis was attempted because the assumption is that orbiting platforms will be available for microgravity research. Decisions on the availability of platforms such as a shuttle or space station are essentially programmatic issues in which microgravity research is only one of many considerations.

\section{RECOMMENDATIONS FOR THE SCIENCE PROGRAM}

The disciplines of microgravity research discussed in this report include fluids and transport, combustion, biological sciences and biotechnology, materials science, and microgravity physics. Materials science is further categorized by constituent subfields: metals and alloys, organic materials and polymers, inorganic crystals, epitaxial layer growth, and ceramics and glasses. The expected degree of scientific success in each of these areas naturally varies:

\section{General Priorities}

- Fluids and transport, combustion, metals and alloys, microgravity physics, and certain areas of biotechnology offer the greatest likelihood for substan- 
tial advances. In these areas, focused experiments can be performed that will expand our knowledge of fundamental phenomena and processes.

- The areas of polymers, ceramics or glasses, inorganic crystals, and epitaxial layer growth, on the other hand, do not at present offer great promise of new advances. Limited support is still warranted, however, for certain fundamental studies.

- Certain areas of the biological sciences and biotechnology should be viewed from a different perspective. Much of the work in these areas is phenomenological. Such research should be pursued to discover the scope and potential for utilizing the microgravity environment.

\section{Areas Recommended for Emphasis}

Major prospects and opportunities in each of five disciplines are summarized in the following sections.

\section{Fluid Mechanics and Transport Phenomena}

Fluid mechanics and transport phenomena play a dual role in the microgravity research program. They stand as distinct disciplinary areas but also appear as themes running through other disciplines. The presentation in this report reflects the role of fluids and transport in the support of other microgravity disciplines.

Most physicochemical transport phenomena are influenced significantly by gravity. As a consequence, unusual behavior is expected in low-gravity environments for many tluid configurations. Also, the reduction of gravitational forces leads to dominance by other forces normally obscured in terrestrial environments, such as surface tension and electrohydrodynamic effects. Basic research is required to understand and describe the unusual characteristics of transport phenomena under low-gravity conditions.

Transport phenomena also play essential roles in many processes that are important to mission-enabling technologies. Predictive models for low-gravity performance and operation of those technologies are frequently inadequate. New models are needed. Strictly empirical approaches are not preferred for lowgravity applications because they are costly and time consuming, and can result in products or systems that are unreliable or inefficient.

Priority should be given to the study of phenomena that are prominent in the low-gravity environment and to those that are critical to space mission-enabling technologies and commercial developments. Among the basic topics that may be studied uniquely with special advantages in the low-gravity environment are the following:

- Surface tension gradient-driven flow's and capillary effects. These are frequently obscured in a terrestrial environment but may become significant or 
dominant in reduced gravity. These topics warrant investigation because they are not well understood and because surface tension-driven flows are ubiquitous for many spaceflight-enabling technologies.

- Multiphase flows. Many processes involve multiphase flows. Gravity imposes a specific orientation on multiphase fluids and structures (e.g., gasliquid, liquid-solid). In a reduced-gravity environment, multiphase flows and associated transport phenomena become significantly different because of the altered orientation of the various phases.

- Diffusive transport processes. At $1 \mathrm{~g}$, multicomponent fluids experience various modes of thermosolutal convection. In addition, there are other effects due to different diffusivities for heat and mass. With reduced-buoyancy convection, these complex interactions can be separated and analyzed. Furthermore, transport effects masked at $1 g$, such as Soret and Dufour phenomena, can become important.

- Colloidal phenomena. At $1 \mathrm{~g}$, the nature of surface and short-range forces, and their consequences in colloidal systems, are often difficult to study because of the complications associated with competing gravitational effects. Microgravity provides an opportunity for study of colloidal systems in which short-range forces are dominant. which can contribute in an important waty to the understanding of these physicochemical interactions.

The above topics appear prominently in many areas of science, including conbustion, biotechnology, materials science, and physics. Therefore, fluid mechanics and transport phenomena should be viewed as a common theme throughout many of the microgravity disciplines.

The following topics deserve attention for both their intrinsic scientific inportance and their applications to space technologies:

- Convective processes at low Reynolds number. Investigation of low Reynolds number flows with density variations, at reduced gravity levels, will reveal the altered nature of transport phenomena in a new range of parametric conditions.

- Transport processes with a phase transition. The modified processes of condensation, evaporation, and boiling in a low-gravity environment with dominant interfacial forces require study.

- Compler materials. Porous, granular, and colloidal media, and foams are complex materials whose structures and functions can differ in microgravity. Research is necessary to understand the behavior of such materials in a lowgravity environment and may also lead to a better understanding of their behavior at $1 \mathrm{~g}$.

- Materials processing. Buoyancy, sedimentation, and interfacial phenomena influence such processing methods as fluidized-bed hydrogenation, electrowinning, and vapor-phase pyrolysis and therefore should be investigated.

- Physical processes in life-and operating-support systems (enabling tech- 
nologies). Some of the effects indicated above apply to processes such as power generation and storage, water purification, oxygen production, and fuel and fluid storage and management.

Numerous parameters govern the topics listed above. Judicious choice of the parameter ranges and configurations will be required to ensure that the information obtained is directly applicable to the reduced-gravity environment.

Clearly, fluids and transport phenomena appear as critical technical issues in many spaceflight-enabling technologies.

\section{Combustion}

Combustion involves fluid mechanics, mass and heat transport, and chemical reaction-all directly or indirectly subject to numerous gravitational effects. The number of parameters to be investigated is large. Several phenomena require long-duration observations and measurements (e.g., smoldering and flame spread).

The following research areas are recommended for emphasis according to rank-order:

1. The highest-priority area, and one of intense practical interest, is that of fires in spacecraft and potential extraterrestrial bases. Microgravity and reducedgravity research is required because of the potential for disaster posed by fires. Much is still unknown about fires in altered gravity conditions. A variablegravity capability would be useful for a full understanding of gravitational effects and for implementation of fire safety measures in spacecraft.

2. In fire research, several subfields of combustion need to be investigated under microgravity conditions. Ignition, flammability limits, smoldering, flame spread, and extinguishment are all deserving of detailed study. Variables should include fuel type and phase, fuel/oxidizer ratio, ambient oxidizer concentration, forced and free convection. ignition source, and extinguishment methodology.

3. Turbulent combustion processes are highly important on Earth but cannot be probed at $1 \mathrm{~g}$ at the small size scales that typically occur. Laboratory scale-up at $1 \mathrm{~g}$ introduces unwanted buoyancy. Reduced gravity would allow a scale-up in overall size without the introduction of major buoyancy effects, thus permitting access to the smallest scales of turbulence that are important to the problem. Here, the Reynolds number based on a forced flow velocity would remain fixed in the scaling process. Even though gravity is not an important parameter for many practical devices, the laboratory study of combustion processes is often impeded at $1 \mathrm{~g}$.

4. Research on laminar premixed and diffusion flames and spray-flow interactions should be conducted, again because of the importance of these processes on Earth. The same problem occurs here as with turbulent flames. That is, scale-up to allow study at $1 g$ introduces unwanted buoyancy effects. 
In addition, other general recommendations are noteworthy.

- Reproducibility must be verified. Many combustion phenomena require a survey of a wide range of parameters. Also, some combustion phenomena are long-duration events. Consequently, an extended orbiting platform capability will be required for many combustion experiments for complete and serious study.

- Relevant to all areas above, ground-based experiments should be undertaken to develop miniaturized diagnostics and experimental apparatus and techniques for performing multiple repetitions of a specific experiment.

\section{Biological Sciences and Biotechnology}

The biological sciences and biotechnology are experimental disciplines that are highly dependent on empirical approaches to the solution of problems and on the continued discovery and development of useful research systems. Unlike many other branches of microgravity science such as fluid dynamics or materials science, there may be no firm foundation of theory, only a limited accumulation of experience. Thus, a reasonably high tolerance for scientific risk (which is clearly distinct from safety risk) should be allowed in investigations in the biological sciences. The science community should be prepared, however, to support research in this area on the basis of its long-term potential and importance, especially its relevance to human spaceflight. Since research on biological topics commonly requires experiments that take a long time to complete, an extended-duration orbiting capability is necessary.

Priorities should be given to scientific issues in biological sciences and biotechnology in the following order:

1. In studies to improve methods of crystallization of macromolecules for use in diffraction studies, additional experiments should focus on defining quantitatively those macromolecular crystal properties and growth mechanisms affected by gravity. There is demonstrated success in this research area and it is recommended for the highest priority.

2. Further experimentation is needed in both terrestrial and microgravity environments to develop new methods, materials, and techniques to exploit the potential of microgravity, where it exists, for improvements in biochemical separations. These separations are important both in terrestrial applications and in materials processing to support human spaceflight.

3. A dedicated effort should be made to evaluate the potential advantages of the microgravity environment for the study of cellular interactions, cell fusion, and multicellular assembly and to identify candidate cell systems that show maximum response to being cultured in such an environment. Efforts should also be made to identify and characterize subcellular mechanisms that sense and mediate responses to the magnitude and direction of gravitational forces. 
4. A systematic effort should be made to identify those cellular and biomolecular processes, structures, assemblies, and mechanisms that may be affected by gravity, and to design and carry out experiments to explore the effects of microgravity on appropriate systems.

\section{Materials Science and Processing}

Metals and Alloys. The microgravity environment provided by an orbiting spacecraft offers new opportunities in the control of melting and solidification processes. Reduction of convective velocities permits, in some cases, more precise control of the temperature and composition of the melt. Body force effects such as sedimentation, hydrostatic pressure, and deformation are similarly reduced. Weak noncontacting forces derived from acoustic, electromagnetic, or electrostatic fields can be used to position specimens while they are being processed, thus avoiding contamination of reactive melts with their containers.

Since many experiments in this field require long time scales for completion, the capability of an extended-duration orbiting platform is needed to obtain full benefits from microgravity research. Topics in the metals and alloys area that would benefit from a focused effort include the following prioritized items:

1. Nucleation control and the achievement of metastable phase states, such as metallic glasses and nanostructures, are areas that could benefit from achieving deep supercooling in the microgravity environment, by elimination of container surfaces, and from the reduction of melt flows due to buoyancy-driven convection.

2. Microgravity experiments on Ostwald ripening and phase coarsening kinetics would add quintitative, fundamental information about the key metallurgical issues of interfacial dynamics during thermal and solutal transport, and the question of microstructure evolution in general.

3. Observations of aligned microstructures processed reproducibly under quiescent microgravity conditions should help to provide well-defined thermal processing limits for polyphase directional solidification of eutectics and monotectics.

4. Studies of the formation of solidification cells and dendrites under welldefined microgravity conditions can add to our expanding knowledge of complex metallurgical pattern formation and, more generally, of the fundamental physics of nonlinear dynamics. Microgravity conditions can be useful in these instances for the pursuit of sophisticated tests of theory and the quantification of metallurgical pattern dynamics.

5. Some thermophysical properties can be measured advantageously in microgravity. Accurate data on such properties, frequently essential for the modeling of metallurgical processes and materials responses, are often not available from standard terrestrial measurements. 
Polymers. Polymers potentially represent the broadest classes of engineered materials, permitting great innovation and precision in design, including control at the molecular level. Although the viscous character of most high polymer melts greatly desensitizes their response to gravitational acceleration, nonetheless some polymer solutions have relatively low viscosities. Moreover, some of these organic systems provide materials with interesting applications in the fields of nonlinear optics and metrology.

- A few initial experiments on the vapor- and solution-phase processing of organic and polymer films in microgravity have shown improved texture and smoothness over terrestrial counterparts, suggesting that this area of research merits further study.

Growth of Inomganic Single Crystals. The microgravity environment will be particularly useful for the study of transport phenomena in the liquids from which bulk crystals are grown, and priority should be given to these studies rather than to the growth of large crystals. Such studies probably require a steady, very-low-gravity environment such as that obtainable in a free-flyer and will provide useful data without requiring growth of bulk crystals. Precise transport data will become particularly useful for fluid dynamics computations. which are rapidly improving for terrestrial melt and solution growth. Any experiments on bulk crystal growth must be judged by their potential to contribute to the scientific understanding of the fundamental processes of crystal growth. The recommendations for this area of research are as follows:

- The design and execution of microgravity experiments that lead to a better fundamental understanding of crystal growth have proved elusive, and the committee recommends against the growth of large inorganic crystals under low gravity. The best approach to understanding the details of such growth will likely derive from fluid dynamical modeling and the modeling of processes at the fluid-solid interface, along with terrestrial studies of crystal growth. This analytical approach may provide the rationale for the growth of benchmarkquality inorganic crystals in microgravity.

- Assuming that some of the versatility of terrestrial experimentation can be achieved in the microgravity environment, there are opportunities for microgravity research that will have an impact on terrestrial bulk crystal growth. Priority should be given to transport studies, including studies of solute and selfdiffusion, heat diffusion, and Soret diffusion. All of these involve the fluid from which crystals are grown, and they are amenable to routine study with repeatedly used apparatus. However, they may require a more stable environment than that provided by the space laboratory. Indeed, since such studies will undoubtedly be sensitive to the acceleration environment, they may also be useful for the study of this environment as a variable in the low-gravity range.

- Precise transport data will become particularly useful since fluid dynam- 
ical computational capabilities (for which these data are required) are improving rapidly for terrestrial melt and solution growth, as well as other industrial processes. Furthermore, transport measurements in industrially important fluids may be an important microgravity application outside the realm of large inorganic crystal growth.

Growth of Epitaxial Layers on Single-Crystal Substrates. During the terrestrial growth of epitaxial layers by vapor deposition, an effort is made to minimize the effects of flow, buoyancy, and boundary layer uniformity by rotating or spinning substrates during the growth process. In addition, attempts are under way to calculate precise flow patterns and resulting growth rates in model systems. The calculations are complex, and it is uncertain whether they will be able to predict occurrences in real systems that are useful for industrial production. Since the manufacture of heterostructures by vapor deposition methods will be accomplished terrestrially, it is not clear whether any relevant data can be obtained solely under microgravity.

- A low priority is recommended for chemical vapor deposition studies under microgravity conditions.

For molecular beam epitaxy (MBE) methods, great gains in purity can, if necessary, be made on Earth, if sufficient attention is given to reducing contamination and increasing pumping speed. The committee concludes that epitaxial layers will be too costly to manufacture in space and that, at a reasonable cost, much improvement in the vacuum environment can be achieved terrestrially.

- Molecular beam epitaxy studies in orbiting vehicles are not appropriate until the limits of ground-based alternatives have been clearly reached.

Ceramics and Glasses. As discussed above, a low-gravity environment will reduce buoyancy-driven convective flows in liquids. Most ceramic synthesis and processing is done at high temperature either by solid-state processes exclusively or by processes in which there are only small amounts of viscous liquid phases. Glasses are formed from high-temperature melts, where the suppression of convective mixing is generally undesirable because convection promotes compositional homogeneity. A second case in which liquids are important to ceramics is in the synthesis of ceramic powders or films from aqueous solutions or colloidal suspensions. Frequently, the requirement is to achieve a high density of nuclei and consequent fine particle sizes of the precipitated powders. Thus, rapid mixing is used, and there is no reason to suppress convection. For these reasons, low-gravity studies are of limited advantage in this discipline.

Nonetheless, a low-gravity environment might be of benefit to ceramics research and development in the following prioritized areas: 
1. There is interesting potential for containerless melting. Processing of ceramics at high temperature requires refractory containers that remain unreactive with the specimen. Availability of a general capability for containerless, high-temperature processing (to at least $1600^{\circ} \mathrm{C}$ ) would allow contaminationfree synthesis of glasses and ceramics, such as those being considered for optoelectronic applications, as well as the study of glass nucleation and crystal growth without heterogeneous nucleation on a container wall.

2. A fundamental study of crystal nucleation and growth in glass melts in microgravity would be interesting. Crystallization is an important process, desirable in glass-ceramics and undesirable in optical glasses, that requires further characterization and understanding.

3. Mass transport and diffusion studies of glass and ceramic melts under microgravity conditions should generate more precise data than those available from terrestrial measurements. One area of research that might be aided by reduction of convection is obtaining accurate data on diffusion in ceramic melts.

4. The suppression of free evaporation from melt surfaces could allow synthesis at higher temperatures than can be performed on Earth.

5. The epitaxial growth of films from solution, including biomimetic synthesis (self-assembling monolayers) of ceramics, should be studied.

\section{Microgravity Physics}

Experiments in this area, with the exception of the general relativity test Gravity Probe-B (GPB), all represent extensions of work that is, or can be. conducted in Earth-based laboratories. Much of the scientific value of the proposed space experiments will depend on the strength of the connection to Earthbound research. Given the long time scale for the ground-based development through flight of a space experiment, there is concern that the scientific goals of the experiment might be bypassed by new developments or by major shifts in the value ascribed to the work.

The topics of microgravity physics fall into two general categories: (1) development of new instruments (e.g., superconducting gyroscopes for the GPB experiment and the mass balance for the equivalence principle test), and (2) preparation and study of unique samples such as uniform fluid free from gravity-induced density gradients or low-density granular materials near a percolation limit. This program can include such important studies as fundamental physics measurements (e.g., verification of the equivalence principle), critical phenomena, dynamics of crystal growth, and low-density aggregate structures.

A recent successful experiment in this area is the Lambda Point Experiment (LPE) that was flown on the shuttle in October 1992 (USMP-1). Analysis of the data indicates an improvement of nearly two orders of magnitude over previous data obtained on Earth. This has been done in an unbiased way, and heat capacity data have been obtained approaching a few nanokelvin of the Lambda point. 
This experiment demonstrates clearly that highly sophisticated experiments involving the most sensitive and advanced instrumentation can be profitably performed in the microgravity environment.

The quality of the microgravity environment must be examined critically in the context of each possible experiment. Minimum acceleration is the most obvious parameter of concern for many of the contemplated experiments; however, the time span over which a high-quality, low-gravity environment can be maintained may be of equal importance. For some experiments, accidental large accelerations (perhaps resulting from sudden movements of personnel or the firing of small thrustors) might destroy the object of study, for example, a lowdensity granular structure.

With regard to the quality of the microgravity environment available for experiments, only the center of the orbiting spacecraft is in true free-fall and then only to the extent that orbital drag effects and other external influences are negligible. In a gravity gradient-stabilized spacecraft, there will be a steady rotation of any experiment about the center of mass of the entire spacecraft once each orbit. For a low Earth orbit, this will result in accelerations on the level of $10^{-7} g$ at a distance 1 meter from the center of mass of the entire orbiting system. On both the space shuttle and the space station. only al few experiments will be located close enough to the center of mass to ensure acccleration levels below $10^{\circ} \mathrm{g}$.

- A number of scientifically meritorious projects, such as the equivalence principle experiment and GPB, will require spaceflight independent of any crewed space facilities.

In the future, we may anticipate a continued requirement for low-temperature facilities in space, since low temperatures are important for the highestresolution measurement techniques, particularly those based on SQUID (Superconducting Quantum Interference Device) technology.

- If a space station is to be a useful contributor to the area of fundamental science, access to liquid-helium facilities will be mandatory.

\section{GENERAL SCIENCE RECOMMENDATIONS FOR MICROGRAVITY RESEARCH}

Following are a number of general science recommendations that are important for microgravity research:

- The reproducibility of results is a crucial element of laboratory science, and flight investigators should be given the opportunity to address reproducibility in their research. Nonetheless, a balance should be established between the reflight opportunities necessary for reproducibility and the flight of experiments that address new scientific issues. 
- The need for scientific judgment, trained observation, and human intervention and participation in certain laboratory microgravity experiments requires a greater use of payload specialists with expertise and laboratory skills directly related to the ongoing experiments. Some flight experiments would also benefit from the direct investigator interaction made possible by teleoperation capabilities, and NASA should support the development and deployment of such techniques in future microgravity experiments.

- As a rule, microgravity experiments should be designed and conducted to provide specific explanations for meaningful scientific questions. Scientific objectives should be clear and specific. In addition, NASA must continue to support ground-based experiments and the development of underlying theories. Theoretical understanding and ground-based experimental results should support the need for microgravity experiments and the likelihood that experimental objectives will be met. Notwithstanding this, exploratory experiments can be valuable in advancing understanding of some complex systems (e.g., biological systems).

- There are scientific reasons to augment microgravity research in certain areas with a variable-gravity capability of extended duration that covers the $\mathrm{mi}$ crogravity to $1-g$ range. In a laboratory science where controlled model experimental systems are essential, the ability to vary an important parameter continuously should be developed whenever feasible. Therefore, if a space station centrifuge is to be developed, it is desirable to evaluate shared utilization for microgravity research.

- NASA should categorize experiments according to their minimum facility requirements to maximize scientific return and cost-effectiveness. Drop towers, aircraft on parabolic trajectories, sounding rockets, and orbiting platforms supply a range of acceleration levels, acceleration spectra, and experimental durations and provide opportunities for human interaction and demonstration of reproducibility of results. Some facilities are more suitable than others for precursor experiments to evaluate instruments and procedures and to demonstrate feasibility.

- General-purpose facilities (versus experiment-specific equipment) should not be imposed on principal investigators if it might degrade the scientific results. Costs are not necessarily lowered when equipment is designed for a wide range of experiments. The scientific benefits could be substantially reduced by the inherent compromises.

- For each flight experiment, the acceleration vector should be accurately measured locally, frequently (or continually), and simultaneously with other experimental measurements. Since the magnitude and direction of the net local acceleration environment can significantly affect experiments, they must be correlated with the primary experimental data. The net acceleration vector varies temporally and with position relative to the spacecraft center and can have major effects even at small magnitudes.

- Materials studied in microgravity experiments should be adequately char- 
acterized on Earth. For some materials, there is a lack of the thermophysical data essential for both experimental design and modeling. These data should be obtained by ground-based research, where possible, or from microgravity measurements, when necessary.

- The qualitative effects of the acceleration environment on certain types of experiments should be studied. These effects are not generally understood and characterized.

\section{FLIGHT OPPORTUNITIES AND CHALLENGES}

The peculiar character of microgravity research as a laboratory science in space requires real-time interactions between scientists and their experiments. This special requirement indicates another role for humans in space, in addition to the traditional use of astronauts for exploration and the deployment and repair of satellites. Moreover, a laboratory-based researcher should be able to carry out a large number of experiments to cover required ranges of the experimental parameters and demonstrate the reproducibility of results. The researcher should be able to modify the apparatus and diagnostics, when necessary, to ensure successful experimentation.

An assortment of facilities is available for research in a low-gravity environment. These range from drop towers and aircraft to orbiting platforms. The duration required for experiments in some of the subdisciplines of microgravity research may exceed the flight time available on some platforms. Additional spacelab missions, particularly those with extended-duration capability, might adequately serve those subdisciplines. For other subdisciplines such as some aspects of biotechnology and materials science, however, longer flight times would provide significant benefits in terms of the quality of scientific yields. Even for those subdisciplines with shorter-duration experiments, the limited number of spacelab flights and the high costs associated with them severely limit the experimenter with regard to demonstration of reproducibility, time for readjusting or repairing equipment, and reflight opportunities. These limitations, inherent to the spacelab system, can be obviated only by the longer flight durations available on a space station or a recoverable satellite. It is important, however, that the design of a space station or recoverable satellite provide a stable microgravity environment that minimizes unacceptable disturbances.

The present microgravity research infrastructure does not readily accommodate the needs of laboratory research. Although drop towers and airplanes flying parabolic trajectories can be used for special or precursor experiments and some materials processing can be done on free-flyers, the spacelab and space station are better suited for microgravity laboratory research. Experience with spacelab for microgravity research, however, indicates that (1) it is likely to take years to develop an experiment that can yield high-quality scientific data and (2) it is an extremely expensive process. 
The time required from the selection of the principal investigator to launch with new hardware is 5 to 6 years for mid-deck (noninteractive) experiments and 6 to 8 years in spacelab. Reflights with minimal modifications to the equipment require 1 year for mid-deck and 2 years for spacelab experiments. Minute elements of an experiment must be documented in detail and subjected to safety tests. Experiments are often delivered up to a full year before launch for integration into the spacelab and then into the orbiter. Because the number of spacelab missions is limited, as many experiments as possible are scheduled for each flight. Thus, the number of experimental runs is limited even if all goes as planned on the mission. As a result, compromises are made that might be favorable for one experiment but not others. For those experiments that suffer in the compromise process, essential science might be sacrificed. Also, because of heavy demand, experimenters are often guaranteed only one flight.

Surmounting the various administrative hurdles confronting an experiment requires interaction with three different NASA centers. The conditions imposed on the experiment by various centers are not always mutually consistent. The principal investigator is often excluded from the discussions. Most of this complexity results from concern for the safety of the crew and spacecraft, and results in long times for experiment development and flight and, consequently, increased cost. Concern for safety in human spaceflight missions should always remain a primary consideration, but safety issues could be fully addressed in a streamlined administrative process that does not compromise the scientific objectives of the mission. The requirement for laboratory science is that human participation in the experimental process be guaranteed, although in some cases, uncrewed flights with remote control of experiments are effective substitutes for physical human presence. Human presence, however, will be necessary for many spaceflight experiments in the foreseeable future.

The entire infrastructure and extensive procedures that are currently extant have been developed essentially for missions in space with purposes other than laboratory science. They should now be reassessed and perhaps modified extensively to take full advantage of the unique microgravity environment.

The biotechnology program has been affected by some confusion concerning its administrative oversight. Biotechnology is tied strongly to other microgravity programs because it shares fluids and transport phenomena as the common scientific theme through which gravity becomes an important parameter. The administrative issue concerns the need for cooperation and coordination between the microgravity research administration and the life sciences administration for research on cellular and subcellular processes and mechanisms.

In summary, the major administrative challenges before NASA in the microgravity research domain are the following: (1) interactions among centers and between centers and headquarters should be simplified and unified; (2) principal investigators should be continually involved with the development of ex- 
periments and: (3) biotechnological research and life sciences programs should be well coordinated.

\section{ADMINISTRATIVE RECOMMENDATIONS}

The following recommendations do not directly address the scientific issues, but rather summarize administrative issues, including some points discussed above, that profoundly affect the quality and quantity of the scientific content of the microgravity program.

- Meaningful interaction should be maintained between the principal investigators and NASA staff during experiment development and integration, and communication among these groups must be continued following flight. It is essential to minimize the overlap of responsibilities in the NASA infrastructure to accomplish this goal.

- It is essential that the overlap of responsibilitics among NASA centers and between centers and headquarters be substantially reduced in order to optimize the influence of the principal investigators and the likelihood of successful experiments.

- Measures should be taken to improve coordination and cooperation between the life sciences administration and the microgravity research administration concerning biological and biotechnological research on cellular and subcellular processes and mechanisms. The strong scientific coupling between biotechnology and other microgravity disciplines through the fluids and transport theme should be recognized in any administrative reorganization.

- All data, including acceleration measurements. should be made available immediately to the principal investigator. Current delays in providing data extend many months beyond the flights. NASA should coordinate the operations of various offices so that priority is given to the processing of experimental data.

- It is necessary to increase substantially the number of ground-based investigations to ensure the future supply of high-quality flight experiments. Consequently, the budget for ground-based research should be increased as a fraction of the entire microgravity program. To the maximum extent feasible, funding for ground-based research in the microgravity program should be protected from temporary budgetary fluctuations.

- In addition to its role in developing flight experiments, the ground-based program can provide important scientific and technical data for other purposes. A ground-based study can therefore be judged successful even if a flight experiment does not result.

- Microgravity research is much broader than the topic of materials processing in space and should be identified as micrograrity research in all official documents, including the federal budget. Microgravity research better describes the activity and its actual and potential accomplishments. Materials processing 
in space characterizes only a fraction of this research activity and promotes a misleading impression of the potential benefits and scope of the program.

- The microgravity sciences program and the commercial development program have a large area of overlap and common interest. Coordination between these programs and an equally stringent review process for each should be fostered by NASA for their mutual benefit.

- NASA should establish mechanisms for continuous, unrestricted submission of research proposals in order to optimize quality and take advantage of scientific advances. Unrestricted submission of research proposals would parallel approaches of other funding agencies and enable continual scientific advance.

- To manage the diverse disciplines in microgravity research, it is necessary to increase the breadth and experience of the scientific staff at NASA headquarters. A rotation of prominent scientists on leave from universities, national laboratories, and industry is one mechanism to be explored. Introduction of active scientists in the administration of the program would be highly beneficial.

- Prompt documentation of experimental results should be required and enforced. Reports of all experiments, including unsuccessful efforts, should be accessible to all interested parties. A concern is that some investigators might not report results because they are proprietary or inconclusive. The lack of an available report could lead to unnecessary duplication of efforts.

- NASA should organize and maintain an accessible archive of microgravity research results. This archive should contain a bibliography of all published scientific papers and reports on microgravity subjects and should preserve the original spaceflight data sets, such as photographs and electronically recorded data.

An additional point of concern is that given the long time scale for the development through flight of a space experiment, there is a real danger that the scientific goals of the experiment might be bypassed by new developments or by major shifts in the value ascribed to the work. There is also the possibility that the principal investigator may lose contact with the field. Several of the above recommendations may be useful in this regard. If NASA can shorten this time frame, it would be beneficial.

\section{REFERENCES}

1. Space Science Board. National Research Council. 1978. Materials Processing in Space. National Academy of Sciences. Washington, D.C.

2. Space Science Board. National Research Council. 1988. Space Science in the Twenty-First Century: Imperatives for the Decades 1995 to 2015. Fundamental Physics and Chemistry. National Acaldemy Press. Washington, D.C

3. Space Studies Board, National Research Council. 1992. Toward a Microgravity Research Strategy. National Academy Press, Washington, D.C. 

PART I

Overview 



\section{Introduction}

Microgravity research is concerned with the identification and description of the effects of reduced gravitational forces on physical, chemical, and biological phenomena. Microgravity research probes a new parameter space where gravitational acceleration no longer is equal to $1 \mathrm{~g}$ and, instead, can approach values that are orders of magnitude lower. Gravity affects a wide variety of scientific areas, some of which have profound implications for space exploration. Scientific disciplines that are affected include fundamental physics, fluid mechanics and transport phenomena, materials science, biological sciences, and combustion. These disciplines are investigated predominantly as laboratory science, which requires the use of controlled, model experiments. Laboratory experiments often require the constant attention and interaction of the experiment$\mathrm{er}$, and their results are validated by their reproducibility. It is its laboratory science character that distinguishes microgravity research from most of the other areas of science commonly recognized as space studies. In this report, these fundamental characteristics of microgravity research are illustrated in many different ways.

As is usually the case in the physical and biological sciences, discoveries are made when a new area or novel parameter space is explored. Microgravity research, despite its relative infancy, is no exception. Increasingly, fundamental processes that were thought to be well understood under terrestrial $(1-g)$ conditions have, in fact, proved to behave in altered and even startlingly unfamiliar ways when observed and measured in reduced-gravity environments. Space experiments in areas such as combustion, fluid flow and transport, phase separation, fundamental physics, and biology have revealed new phenomena and have 
demonstrated new and occasionally unpredicted behavior. Indeed, many commonly experienced phenomena can be dramatically altered in a low-gravity setting: for example, the dynamic behavior of gas bubbles in fluids, the sedimentation of particles, the characteristics of the flame of a burning candle, and the behavior of fluids within and outside their containers. This modified behavior will profoundly affect the technology for handling liquids and gases in reducedgravity environments. Nevertheless, virtually all scientific experimental research in these areas has taken place in the terrestrial setting of $I g$.

Microgravity science is neither a homogeneous nor a distinct discipline. It has evolved gradually over the past two decades into a range of multidisciplinary space research activities in which the unifying features are basic and applied studies of gravitational interactions with fluids and a variety of condensed states, and with transport phenomena in physical, chemical, and biological systems. In fact, the fluid mechanics and transport sciences constitute the core science content of microgravity studies. Therefore, in considering new and important directions for microgravity research, considerable emphasis is given in this report to the study of transport phenomena. These phenomena are strongly gravity dependent; they have extensive applications to space systems engineering; and they play a pivotal role in many important modern technologies.

\section{THE MICROGRAVITY ENVIRONMENT}

The availability of orbiting laboratories, both crewed and uncrewed, has provided access to an environment in which processes can be studied under sustained levels of reduced gravity. The term microgravity is itself something of a misnomer. Because the gravitational force is infinite in extent, low-gravity conditions can be achieved only at substantial distances from any massive object. In the current context, however, microgravity describes the acceleration conditions attending free-fall, that is, the acceleration sensed within an inertial reference frame that is at rest with respect to the local environment but accelerating toward the center of the Earth. Such conditions occur for limited periods in drop towers and in aircraft executing parabolic orbits. Spacecraft and satellites in near-Earth orbit also operate at reduced gravity levels (down to $10^{-6} \mathrm{~g}$ ) and have the additional advantage of providing much longer times for exploiting this environment.

The principal characteristic of low-gravity environments is that the net gravitational body forces are reduced substantially. This leads to decreases in hydrostatic pressure, buoyancy-driven flows, and rates of sedimentation. Reduction of the gravitational force, or weight, permits other forces, such as that due to surface tension, to become important, if not dominant. An immediate consequence is that under microgravity conditions, multicomponent and multiphase fluid and other fluid-solid system behaviors (e.g., mixing, separation, and interfacial phenomena) are significantly altered. Many other fluid transport phenomena involving heat and 
mass transfer are also influenced by the reduction of gravity. There is increasing recognition that transport phenomena are of central importance in a wide range of physical, chemical, and biological systems that play major roles in many terrestrial and space-based technologies. Specifically, if NASA's long-range program in space science, exploration, and transportation is ever to be realized, it is essential to obtain a comprehensive understanding of low-gravity fluid phenomena and their consequences for extraterrestrial processing.

\section{PERCEPTIONS AND REALITIES OF MICROGRAVITY RESEARCH}

The history of microgravity research is not extensive-the field is barely a few decades old. Furthermore, only a limited number of microgravity experiments and samples have been studied to date, and the accumulated microgravity laboratory experience known to the U.S. scientific community amounts to less than 1000 hours of in-orbit time. Several recent NASA shuttle missions have been dedicated to laboratory science in microgravity (USMP-2 and IML-2) and are expected to yield extensive data and sample returns. Some information about the scientific results from spaceflight is provided in Chapters 3 through 7 . At present, however, all of the data have not been fully analyzed and reported.

Nevertheless, there have been some notable successes. For example, the Lambda Point Experiment (LPE) was flown on the shuttle in October 1992 (USMP-1). Analysis of the data indicates that an improvement of nearly two orders of magnitude over previous data obtained on Earth has been achieved. This was done in an unbiased way, and heat capacity data approaching within a few nanokelvins of the Lambda point were obtained. This experiment gives a clear demonstration that highly sophisticated experiments involving the most sensitive and advanced instrumentation can be performed reliably in the microgravity environment.

For many years, microgravity research was perceived as focused primarily on materials processing. Indeed, the microgravity line item in NASA's budget has even been titled "Materials Processing in Space." Although NASA's microgravity research program certainly includes research conducted on materials science and materials processing, the reality is that this program is much broader in its scope. Specifically, current microgravity research, viewed as a laboratory science, is also broadly directed toward fundamental studies in physics, chemistry, and biology. Access to prolonged periods in space, as well as to other shortduration, ground-based microgravity facilities, is beginning to provide researchers with the opportunity to apply the methods of the physical and biological sciences to a new regime of low-gravity experiments. Thus, the acceleration of gravity is becoming a meaningful, independent, experimental parameter. This approach is analogous to that employed for more than a century in low-temperature physics, in which diverse phenomena are studied in a cryogenic regime 
where temperature is the primary independent variable that may be set arbitrarily close to zero.

In summary, microgravity research involves strong elements of traditional laboratory science, in contrast with the observational science that constitutes most of the NASA space science program. Experiments often involve controlled model systems and exacting parameter settings. Numerous experimental conditions must be explored, and repeat experiments must be conducted for each investigation in order to understand the phenomena of interest and establish, according to scientific standards, the validity and reproducibility of the data. By contrast, however, the U.S. microgravity research program has produced in its limited experience of in-orbit experimental time only a few score samples and sets of data that meet high scientific standards. This situation is not surprising in view of the fact that humans have been able to make observations in the absence of gravity for less than a quarter of a century and that serious laboratory experiments have been feasible in space for only about 15 years. The decade of the 1990 s represents the first phase of experimentation in microgravity and the potential advent of a true laboratory science in space.

A number of previous National Research Council (NRC) reports have evaluated the field of microgravity research at different stages of its development. The 1978 report Materials Processing in Space (the "STAMPS" report)' provided guidance during the program's early years, whereas Space Science in the Twenty-First Century: Imperatives for the Decades 1995 to $2015^{2}$ attempted to identify future research opportunities. More recently, the 1992 report Toward a Microgravity Research Strategy ${ }^{3}$ began to lay a foundation for a more mature research program, and the current report is a continuation of that effort. More detailed descriptions of these studies can be found in the preface.

Although this report does not provide a complete strategy for microgravity research, it does present some of the important elements of a strategy, including the following:

- A summary of the current state of knowledge of microgravity science;

- A discussion of some of the fundamental questions to be answered;

- A presentation of the goals of the field of microgravity science;

- The science objectives within each discipline;

- An evaluation of the potential for microgravity research to provide advances within each discipline;

- The experimental requirements for achieving the science objectives of each discipline:

- A description of the other resources required for a successful microgravity science program; and

- A limited prioritization of research topics within each discipline.

The two aspects of a strategy for microgravity research that are not presented in this report are (1) a prioritization of microgravity research objectives across 
disciplines and (2) a cost-benefit analysis of anticipated microgravity results. Experience has shown how difficult it is to set research priorities, even within a single homogeneous science discipline. Reaching agreement on priorities for microgravity research relative to all other science research was judged so unlikely that it was not attempted in this report. The other practical reason that stricter priorities were not set for microgravity research stems from the nature of shuttle flights. Frequently, payloads are assigned to a flight because their requirements for space, power, and so on, fit what is available, and scientific priority per se is less important. No cost-benefit analysis was attempted because the assumption is that orbiting platforms, launched for other purposes, will be available for microgravity research. Decisions on the availability of platforms such as the shuttle or space station are essentially programmatic issues in which microgravity research is only one of many considerations. Furthermore, the cost-benefit of a microgravity program has not been compared to the cost-benefit of experiments on different subjects in the terrestrial environment. Experiments that can be performed adequately under terrestrial gravity conditions, however, are not given a priority for spaceflight in this report. Finally, although the value and need for human intervention capabilities and long-duration flights are noted repeatedly in this report, nothing herein should necessarily be interpreted as advocating or opposing any specific NASA spacecraft or space station design initiative.

The remainder of Part $\mathbf{I}$ of this report discusses current research opportunities and presents the major conclusions and recommendations of the report. Specific conclusions and recommendations for each of the five disciplines of microgravity research are discussed first and are followed by general recommendations and conclusions that prioritize research issues and provide some general guidance for administrative policy and procedures. Part II presents a status chapter for each of the five scientific disciplines. Chapter 3 discusses the status of fluid and transport science and also serves to introduce the other science sections because fluid flow is a theme common to most of the other areas. Some discussion concerning facilities and research administration is presented in Part III, which covers programmatic issues.

\section{REFERENCES}

1. Space Science Board, National Research Council. 1978. Materials Processing in Space. National Academy of Sciences, Washington, D.C.

2. Space Science Board, National Research Council. 1988. Space Science in the Twenty-First Century: Imperatives for the Decades 1995 to 2015. Fundamental Physics and Chemistry. National Academy Press, Washington, D.C.

3. Space Studies Board, National Research Council. 1992. Toward a Microgravity Research Strategy. National Academy Press, Washington, D.C. 


\section{2 \\ Recommendations and Conclusions}

\section{INTRODUCTION}

The microgravity environment presents unique opportunities to perform important experimental laboratory research in a number of fundamental and applied areas. The five scientific disciplinary areas that can benefit in varying degrees are (1) fluid mechanics and transport phenomena, (2) combustion, (3) biological sciences and biotechnology, (4) materials science, and (5) microgravity physics. There are important scientific questions in these disciplines that cannot be addressed by experiments at normal Earth gravity. This provides a major impetus for the microgravity research program.

Microgravity research is also necessary for, and fundamental to, the exploration of space. Fluid and thermal systems and materials processes that are essential to mission-enabling technologies for extended space operations will generally behave differently under reduced gravity conditions. Microgravity research opportunities will frequently be driven by the technological needs of NASA's programs. It is well understood that engineering, applied science, and fundamental science are interconnected. Some of the obvious engineering issues that should influence the microgravity research program are the ignition, propagation, and extinction of spacecraft fires; the fluid dynamics and transport processes associated with the handling, storage, and use in space of water, waste, foods, fuels, air environments, and contaminants; the handling, joining, and reshaping of materials in space; and the dynamics and chemistry of mining and refining of resources in non-Earth environments.

The committee reaffirms the findings of the previous and first report, 
Toward a Microgravity Research Strategy, ${ }^{1}$ of the Committee on Microgravity Research that there is little potential for a successful program to develop manufacturing on a large scale in space for the purpose of returning high-quality, economically viable products to Earth. The cost of transporting raw materials into space and products back to Earth will generally be much too high to be economical. Limited amounts of certain products, however, might usefully be made in space for scientific study.

A set of recommendations has been developed for the microgravity sciences that has several salient features. Five science areas are recommended for longterm support with a balance of support among these areas-fluid mechanics and transport phenomena, combustion, biological sciences and biotechnology, microgravity physics - and within materials science, the subarea of metals and alloys. Fields that will benefit significantly less from a microgravity environment are polymers, epitaxial layer growth on single-crystal substrates, large single inorganic crystal growth, and ceramics and glasses. It is noted that the common and major theme among the five recommended areas is that of fluid mechanics and transport phenomena. The committee's recommendations emphasize the development of research opportunities, modification of NASA policies and procedures, and interactions between NASA and the investigators that will provide an environment necessary for first-class laboratory science. The importance of a strong ground-based program is also stressed. The roles of microgravity research are discussed both for the advancement of general scientific knowledge and for the development of enabling technologies for space missions. The lists of scientific recommendations identify the obvious opportunities for immediate and significant scientific impact.

A number of points were considered in arriving at the recommendations for each discipline. The importance and compelling nature of the scientific questions and the perceived impact on the field were given greatest weight and established the context for further recommendations. Scientific questions and objectives, however, were examined further in terms of their requirement for, and probable benefit from, a microgravity environment. Clearly, the priority of a given objective was reduced if alternative or superior approaches were available.

The type of microgravity facility required for the investigations and the quality of the microgravity environment required were also considered. These considerations addressed experimental duration, maximum allowable acceleration level, permissible radiation levels, degree of human or robotic interaction, and complexity of diagnostics. The following questions were asked: Have the issues been explored sufficiently in conventional laboratories on Earth, have adequate theoretical and computational analyses been performed, and are models for the process available? Finally, the resource requirement and costs were considered in the broad context of the current, and anticipated, microgravity research program.

In addition to scientific considerations, means are recommended to improve 
the scientific quality and increase the efficiency of microgravity research. As a result, suggestions are included that relate to instrument design and implementation, procedural matters, and interactions among the space agencies and the investigator communities.

The research areas discussed in this report are subsets of much broader disciplines. Only a small fraction of the total activities in each occurs within the microgravity program, and no attempt has been made to evaluate fully or prioritize all of the research in a particular discipline. Only the microgravity component of each discipline is addressed in detail. Furthermore, the cost-benefit of a microgravity program has not been compared to the cost-benefit of experiments on similar subjects in the terrestrial environment. Experiments that can be performed adequately at Earth gravity are not recommended for spaceflight.

Further, because of the limited results available from experiments that have been flown in space, the recommendations of this report cannot be highly detailed or exclusive. There are a number of subjects that require further exploratory investigation before detailed objectives can be defined. Some areas, however, can be identified as more promising than others. This report also does not address the NASA commercial program or international programs in microgravity research. These topics will be addressed in the future by this committee.

In summary, the recommendations in this report emphasize the contribution of microgravity research to the advancement of science and technology in general and of space exploration in particular. The report also deemphasizes the concept of manufacturing products in space for their return to Earth. Adjustments in NASA's structures, policies, and procedures for conducting laboratory science in space are required by these recommendations. Finally, priorities are suggested for topics within certain microgravity science disciplines and subdisciplines.

The duration of experiments, the regime of parameters available to experimenters, and the ability to demonstrate reproducibility of results in many microgravity experiments create the need for extended-duration orbiting platforms. The recommendations have value, however, whether such platforms become available or the microgravity program continues only with existing facilities.

The findings and recommendations of this report are consistent with the preliminary statements of the first report of this committee, Tow'ard a Microgravity Research Stategy. This second report is broader in scope and includes substantially greater detail in analysis and recommendations.

\section{AREAS RECOMMENDED FOR EMPHASIS IN MICROGRAVITY RESEARCH}

Microgravity science is highly interdisciplinary and broad. The major prospects and opportunities in each of the major disciplines are summarized in this section. Limitations of opportunities are also discussed. 


\section{Fluid Mechanics and Transport Phenomena}

Fluid mechanics and transport phenomena are influenced significantly by gravity. As a consequence, different behaviors may be expected for many fluid configurations in a microgravity environment, both with and without heat and mass transfer. Also, the reduction of gravitational body forces leads to dominance by other forces normally obscured in terrestrial environments such as surface tension and electrical forces. Because fluid mechanics and transport processes are involved in most of the areas of microgravity research, they represent a common theme for much of the subject. Basic research is required to understand and describe the characteristics of transport phenomena under lowgravity conditions.

Fluid mechanics and transport phenomena also play an essential role in many space-based technologies. Space system designers will be challenged to develop new enabling technologies and critical concepts that involve fluid mechanics and transport phenomena in low-gravity environments. Unfortunately, predictive models for the low-gravity performance and operation of those technologies are often inadequate. In fact, some researchers believe that useful predictive models do not exist. Strictly empirical approaches are not preferred for low-gravity applications because they are costly and time consuming, and can result in products or systems that are neither reliable nor efficient.

Priority should be given to the study of phenomena that are unique to the low-gravity environment and to those that are critical to space mission-enabling technologies and commercial developments. Among the basic topics that may be studied uniquely in the low-gravity environment are the following:

- Surface tension gradient-driven flows and capillary effects. These are frequently obscured in a terrestrial environment but may become significant or dominant in reduced gravity. These topics warrant investigation because they are not well understood and because surface tension-driven flows are ubiquitous for many mission-enabling technologies.

- Multiphase flows. Many processes involve multiphase flows. Gravity imposes a specific orientation on multiphase fluids, structures, and organizations (e.g., gas-liquid, liquid-solid). In a reduced-gravity environment, orientation will be much less important; flows and associated transport phenomena become significantly different.

- Diffusive transport processes. At $1 \mathrm{~g}$, multicomponent fluids experience various modes of thermosolutal convection. In addition, there are other effects due to different diffusivities for heat and mass. With reduced-buoyancy convection, the complex interactions can be separated and analyzed. Furthermore, transport effects masked at $1 \mathrm{~g}$, such as Soret and Dufour phenomena, can be come important.

- Colloidal phenomena. At $1 \mathrm{~g}$, the nature of surface and short-range forces, and their consequences in colloidal systems, are often difficult to study because of the complications associated with competing gravitational effects. 
Microgravity provides an opportunity for study of colloidal systems in which short-range forces are dominant, and this can contribute in an important way to understanding these physicochemical interactions.

The above topics are important to biotechnology, combustion, materials science, and other areas of science.

The following topics deserve attention not only for their intrinsic scientific importance but also to provide a knowledge base for space technologies and applications:

- Convective processes at low Reynolds number. Investigation of low Reynolds number flows with density variations at reduced gravity levels will reveal the altered nature of transport phenomena in a new range of parametric conditions.

- Transport processes with a phase transition. The modified processes of condensation, evaporation, and boiling in a low-gravity environment with dominant interfacial forces require study.

- Complex materials. Porous, granular, and colloidal media, and foams are complex materials whose structures and functions can differ in microgravity. Research is necessary to understand the behavior of such materials in a lowgravity environment and may also lead to a better understanding of their behavior at $1 \mathrm{~g}$.

- Materials processing. Buoyancy, sedimentation, and interfacial phenomena influence such processing methods as fluidized-bed hydrogenation, electrowinning, and vapor-phase pyrolysis and therefore should be investigated.

- Physical processes in life-and operating-support systems. Some of the effects indicated above apply to processes such as power generation and storage. water purification, oxygen production, and fuel and fluid storage and management.

Numerous parameters govern the topics listed above. Judicious choice of the parameter ranges and configurations will be required to ensure that the information obtained is directly applicable to the reduced-gravity environment.

\section{Combustion}

Combustion involves fluid mechanics, mass and heat transport, and chemical reaction--all directly or indirectly subject to numerous gravitational effects. The number of parameters to be investigated is large. Several phenomena (e.g., smoldering and flame spread) require long-duration observations and measurements. Furthermore, reproducibility is an issue. Recommendations concerning equipment and facilities include the following:

- An extended orbiting platform capability will be required for many combustion experiments.

- Ground-based combustion experiments must be undertaken to develop miniaturized diagnostics and experimental apparatus and techniques for perform- 
ing multiple repetitions of a specific experiment. Experimental space is at a premium in microgravity research, and because of the many variables in combustion and the difficulties in achieving reproducibility, assurance of data quality is needed.

The following areas of emphasis are recommended in order of priority:

1. The highest-priority area, and one of intense practical interest, is that of fires in spacecraft and potential extraterrestrial bases. Microgravity and reducedgravity research is required because of the potential for disaster posed by fires. Much is still unknown about fires in altered gravity conditions. A variablegravity capability is required for a full understanding of gravitational effects and for implementation of fire safety measures in spacecraft.

2. In fire research, several subfields of combustion need to be investigated under microgravity conditions. Ignition, flammability limits, smoldering, flame spread, and extinguishment are all deserving of detailed study. Variables must include fuel type and phase, fuel/oxidizer ratio, ambient oxidizer concentration, forced and free convection, ignition source, and extinguishment methodology.

3. Turbulent combustion processes are highly important on Earth but cannot be probed at $1 g$ at the small size scales that typically occur. Reduced gravity would allow a scale-up in overall size without the introduction of major buoyancy effects, thus permitting access to the smallest scales of turbulence that are important to the problem. Here, the Reynolds number based on a forced flow velocity would remain fixed in the scaling process.

4. Research on laminar premixed and diffusion flames and spray-flow interactions should be conducted, again because of the importance of these processes on Earth. The same problem occurs here as with turbulent flames. Even though gravity is not an important parameter for many practical devices, the study of fundamental combustion processes is often impeded at $1 \mathrm{~g}$ because attempts at scale-up introduce unwanted buoyancy.

\section{Biological Sciences and Biotechnology}

The biological sciences and biotechnology are experimental disciplines that are highly dependent on empirical approaches to the solution of problems and on the continued discovery and development of useful research systems. Unlike many other branches of microgravity science, such as fluid dynamics or materials science, there may be no firm foundation of theory, only a limited accumulation of experience. Thus, a reasonably high tolerance for scientific risk (which is clearly distinct from safety risk) should be allowed in investigations in the biological sciences. The science community should be prepared, however, to support research in this area on the basis of its long-term potential and importance, especially its relevance to human spaceflight. In addition, since research on 
biological topics commonly requires experiments that take a long time to complete, an extended-duration orbiting capability is necessary.

The following topics deserve priority in the order listed. The first two topics have some demonstrated successes and therefore the likelihood of further success. The last two topics are exploratory.

1. In studies to improve methods of crystallization of macromolecules for use in diffraction studies, additional experiments should focus on defining quantitatively those macromolecular crystal growth mechanisms affected by gravity. Direct, high-resolution observation of the crystal growth process by a variety of optical techniques and precise monitoring of ambient parameters such as temperature and $\mathrm{pH}$ should be included. Ideal test systems should be identified and carefully delineated.

2. Further experimentation is needed in both terrestrial and microgravity environments to develop new methods, materials, and techniques to exploit the potential of microgravity, where it exists, for improvements in biochemical separations. These separations are important both in terrestrial applications and in materials processing to support human spaceflight.

3. A dedicated effort should be made to evaluate the potential advantages of the microgravity environment for the study of cellular interactions, cell fusion, and multicellular assembly and to identify candidate cell systems that show maximum response to being cultured in such an environment. This effort should follow and build on the previous effort to identify mechanisms, processes, structure, and assemblies. Efforts should also be made to identify and characterize subcellular mechanisms that sense and mediate responses to the magnitude and direction of gravitational forces.

4. A systematic effort should be made to identify those cellular and biomolecular processes, structures, assemblies, and mechanisms that may be affected by gravity, and to design and carry out experiments to explore the effects of microgravity on appropriate systems. A comprehensive database should be compiled of cell types. organelles, and other assemblies, and their responses to microgravity environments. This systematic approach has substantial potential impact on our understanding of the underlying processes in the microgravity environment. It should therefore have an impact on the knowledge base for space exploration.

\section{Materials Science and Processing}

\section{Metals and Allow}

Carefully designed and scientifically well-conceived experiments on metals and alloys are needed to produce high-quality data and materials uniquely derived from microgravity research. Such experiments are not useful unless they produce results that cannot be obtained from terrestrial studies. Since many 
experiments in this field require long time scales for completion, the capability of an extended-duration orbiting platform is needed to obtain full benefits from microgravity research. Topics in the metals and alloys area that might benefit from focused microgravity research include the following items listed in order of general priority:

I. Nucleation kinetics and the achievement of metastable phase states, such as metallic glasses and nanostructures, are areas that could benefit from achieving deep supercooling in the microgravity environment, from the elimination of container surfaces, and from the reduction of melt flows due to buoyancy-driven convection.

2. Microgravity experiments on Ostwald ripening and phase coarsening kinetics would add quantitative, fundamental information about the key metallurgical issues of interfacial dynamics during thermal and solutal transport, and the question of microstructure evolution in general.

3. Observations of aligned microstructures processed reproducibly under quiescent microgravity conditions should help to provide well-defined thermal processing limits for polyphase directional solidification of eutectics and monotectics. These comprise wide classes of technologically important alloys and composites.

4. Studies of the formation of solidification cells and dendrites under welldefined microgravity conditions can add to our expanding knowledge of complex metallurgical pattern formation and, more generally, of the fundamental physics of nonlinear dynamics. Such studies, to be successful, require the most demanding control of temperature, thermal gradients, growth speed, alloy composition, and other such parametric variables. Microgravity conditions can be useful in these instances for the pursuit of sophisticated tests of theory and the quantification of metallurgical pattern dynamics.

5. Some thermophysical properties can be measured advantageously in microgravity. Accurate data on these properties, frequently essential for the modeling of metallurgical processes and materials responses, are often not available from standard terrestrial measurements.

\section{Polymers}

Polymers potentially represent the broadest classes of "engineered" materials, permitting great innovation and precision in their design, including control at the molecular level.

- Although the viscous character of most high polymer melts greatly desensitizes their response to gravitational acceleration, initial experiments in some areas of vapor- and solution-phase processing of organic and polymer films in microgravity show improved texlure and smoothness over terrestrial counterparts, suggesting that this area of research merits further study. 


\section{Growth of Inorganic Single Crystals}

The microgravity environment will be particularly useful for the study of transport properties in liquids from which bulk crystals are grown, and priority should be given to these studies. Such studies probably require a steady, verylow-gravity environment (less than $10^{-6}$ ) such as that obtainable in a free-flyer and will provide useful data without requiring growth of bulk crystals. Precise transport data will be particularly useful for fluid dynamics computations, which are rapidly improving for terrestrial melt and solution growth. Any experiments on bulk crystal growth must be judged by their potential to contribute to the scientific understanding of the fundamental processes of crystal growth. The recommendations in this area of research are as follows:

- The design and execution of microgravity experiments that lead to a better fundamental understanding of crystal growth have proved elusive, and the committee recommends against the growth of large inorganic crystals under low gravity. The best approach to understanding the details of such growth will likely derive from fluid dynamical modeling and the modeling of processes at the fluid-solid surface, along with terrestrial studies of crystal growth. This analytical approach may provide the rationale for the growth of benchmarkquality inorganic crystals in microgravity.

- Assuming that some of the versatility of terrestrial experimentation can be achieved in the microgravity environment, there are opportunities for microgravity research that will have an impact on terrestrial bulk crystal growth. Priority should be given to transport studies, including studies of solute and selfdiffusion, heat diffusion, and Soret diffusion. All of these are studies of the fluid from which crystals are grown and are amenable to routine study with repeatedly used apparatus. However, they may require a more stable environment than that provided by the space laboratory. Indeed, since such studies will undoubtedly be sensitive to the acceleration environment, they may also be useful for the study of this environment as a variable in the low-gravity range.

- Precise transport data will become particularly useful since fluid dynamical computational capabilities are improving rapidly for terrestrial melt and solution growth, as well as other industrial processes. These kinds of data will be required for such computations. Furthermore, transport measurements in industrially important fluids may be an important microgravity application outside the realm of large inorganic crystal growth.

\section{Growth of Epitaxial Layers on Single-Crystal Substrates}

During the terrestrial growth of epitaxial layers by vapor deposition, an effort is made to minimize the effects of flow, buoyancy, and boundary layer uniformity by rotating or spinning substrates during the growth process. In addition, attempts are under way to calculate precisely flow patterns and result- 
ing growth rates in model systems. The calculations are complex, and it is not certain whether they will be able to predict occurrences in real systems that are useful for industrial production. Since the manufacture of heterostructures by vapor deposition methods will be accomplished terrestrially, it is not clear whether any relevant data can be obtained solely under microgravity conditions.

- A low priority is recommended for chemical vapor deposition studies under microgravity conditions.

For molecular beam epitaxy methods, great gains in purity can be made on Earth provided sufficient attention is given to reducing contamination and increasing pumping speed.

- Epitaxial layers will be too costly to manufacture in space. At a reasonable cost, much improvement in the vacuum environment can be achieved terrestrially. Studies in orbiting vehicles are not in order until the limits of groundbased alternatives have clearly been reached.

\section{Ceramics and Glasses}

Most ceramic synthesis and processing is achieved at high temperatures either by solid-state processes exclusively or by those processes in which there are only small amounts of viscous liquid phases. Glasses are formed from hightemperature melts (the viscosity of $\mathrm{SiO}_{2}$ at $1700^{\circ} \mathrm{C}$ is about $10^{7}$ poise) where the suppression of convective mixing is generally undesirable because convection promotes compositional homogeneity. A second case in which liquids are important to ceramics is in the synthesis of ceramic powders or films from aqueous solutions or colloidal suspensions. Frequently, the requirement is for a high density of nuclei and consequent fine particle sizes of the precipitated powders. Thus, rapid mixing is used, and there is no reason to suppress convection. For these reasons, low-gravity studies are of limited advantage in this discipline.

Nonetheless, a low-gravity environment might be of benefit to ceramics research and development in the following prioritized areas:

1. There is interesting potential for containerless melting in microgravity. Processing of ceramics at high temperatures requires refractory containers that remain unreactive with the specimen. Availability of a general capability for containerless, high-temperature processing (to at least $1600^{\circ} \mathrm{C}$ ) would allow contamination-free synthesis of glasses and ceramics, such as those being considered for optoelectronic applications. Containerless melting also might allow study of nucleation and crystal growth without heterogeneous nucleation on a container wall.

2. A fundamental study of crystal nucleation and growth in glass melts in microgravity would be interesting. Crystallization is an important process, de- 
sirable in glass-ceramics and undesirable in optical glasses. that requires further characterization and understanding.

3. Mass transport and diffusion studies of glass and ceramic melts under microgravity conditions should generate more precise data than those available from terrestrial measurements. One area of research that might be aided by reduction of convection is obtaining accurate data on diffusion in ceramic melts.

4. The suppression of free evaporation from melt surfaces could allow synthesis at higher temperatures than can be performed on Earth.

5. The epitaxial growth of films from solution, including biomimetic synthesis (self-assembling monolayers) of ceramics, should be studied.

\section{Microgravity Physics}

Experiments in this area, with the exception of the general relativity test, Gravity Probe-B (GPB), all represent extensions of work that is currently conducted or can be conducted in Earth-based laboratories. Much of the scientific value of the proposed space experiments will depend on the strength of the connection to Earth-bound research.

The topics of microgravity physics fall into two general categories: (1) development of new instruments (e.g., superconducting gyroscopes for the GPB experiment and the mass balance for the equivalence principle test), and (2) preparation and study of unique samples such as uniform fluids free from gravity-induced density gradients or low-density granular materials near a percolation limit. This program can include such important studies as fundamental physics measurements (e.g., verification of the equivalence principle), critical phenomena, dynamics of crystal growth, and low-density aggregate structures.

The quality of the microgravity environment must be examined critically in the context of each possible experiment. Minimum acceleration is the most obvious parameter of concern for many of the contemplated experiments; however, the time span over which a high-quality, low-gravity environment can be maintained may be of equal importance. For some experiments, accidental large accelerations (perhaps resulting from sudden movements of personnel or the firing of small thrustors) might destroy the object of study, for example, a lowdensity granular structure.

With regard to the quality of the microgravity environment available for experiment, only the center of the orbiting spacecraft is in true free-fall and then only to the extent that orbital drag effects and other external influences such as solar wind and radiation are negligible. In a gravity gradient-stabilized spacecraft, there will be a steady rotation of any experiment about the center of mass of the entire spacecraft once each orbit. For a low Earth orbit, this will result in accelerations on the $10^{-7}-g$ level at a distance 1 meter from the center of mass of the entire orbiting system. On both the space shuttle and the space station, only 
a few experiments will be located close enough to the center of mass to ensure acceleration levels below the $10^{-6}-g$ level.

- A number of scientifically meritorious projects, such as the equivalence principle experiment and GPB, will require spaceflight independent of any crewed space facilities.

In the future, we may anticipate a continued requirement for low-temperature facilities in space, since low temperatures are important for the highestresolution measurement techniques, particularly those based on SQUID (Superconducting Quantum Interference Device) technology.

- If a space station is to be a useful contributor to the area of fundamental science, access to liquid-helium facilities will be mandatory.

\section{GENERAL SCIENCE RECOMMENDATIONS FOR MICROGRAVITY RESEARCH}

Following are a number of general science recommendations that are important for microgravity research:

- The reproducibility of results is a crucial element of laboratory science, and flight investigators should be given the opportunity to address reproducibility in their research. Nonetheless, a balance should be established between the reflight opportunities necessary for reproducibility and the flight of experiments that address new scientific issues.

- The need for scientific judgment, trained observation, and human intervention and participation in certain laboratory microgravity experiments requires a greater use of payload specialists with expertise and laboratory skills directly related to the ongoing experiments. Some flight experiments would also benefit from the direct investigator interaction made possible by teleoperation capabilities, and NASA should support the development and deployment of such techniques in future microgravity experiments.

- As a rule, microgravity experiments should be designed and conducted to provide specific explanations for meaningful scientific questions. Scientific objectives should be clear and specific. In addition, NASA must continue to support ground-based experiments and the development of underlying theories. Theoretical understanding and ground-based experimental results support the need for microgravity experiments and the likelihood that experimental objectives will be met. Notwithstanding this, exploratory experiments can be valuable in advancing understanding of some complex systems (e.g., biological systems).

- There are scientific reasons to augment microgravity research in certain areas with a variable-gravity capability of extended duration. In some problem areas, the magnitude of the gravity vector is an important experimental variable in the microgravity to $1-g$ range. In a laboratory science where controlled model 
experimental systems are essential, the ability to vary an important parameter continuously should be developed whenever feasible. Therefore, if a space station centrifuge is to be developed, it is desirable to evaluate shared utilization for microgravity research.

- NASA should categorize experiments according to their minimum facility requirements to maximize scientific return and cost-effectiveness. Drop towers, aircraft on parabolic trajectories, sounding rockets, and orbiting platforms supply a range of acceleration levels, acceleration spectra, and experimental durations and provide opportunities for human interaction and demonstration of reproducibility. Some facilities are more suitable than others for precursor experiments to evaluate instruments and procedures and to demonstrate feasibility.

- General-purpose facilities (versus experiment-specific equipment) should not be imposed on principal investigators if it might degrade the scientific results. Costs are not necessarily lowered when equipment is designed for a wide range of experiments; moreover, the scientific benefits could be substantially reduced by the inherent compromises.

- For each flight experiment, the acceleration vector should be accurately measured locally, frequently (or continually), and simultaneously with other experimental measurements. Since the magnitude and direction of the net local acceleration environment can significantly affect experiments, they must be correlated with the primary experimental data. The net acceleration vector varies temporally and with position relative to the spacecraft center and can have major effects even at small magnitudes.

- Materials studied in microgravity experiments should be adequately characterized on Earth. For some materials, there is a lack of the thermophysical data essential for both experimental design and modeling. These data should be obtained by ground-based research where possible or from microgravity measurements if necessary.

- The qualitative effects of the acceleration environment on certain types of experiments should be studied. These effects are not generally understood and characterized.

\section{ADMINISTRATIVE RECOMMENDATIONS}

The following recommendations do not directly address scientific issues but instead summarize administrative issues that profoundly affect the quality and quantity of the science content of the microgravity program.

- Meaningful interaction should be maintained between the principal investigators and NASA staff during experiment development and integration, and communication among these groups must be continued following flight. It is essential to minimize the overlap of responsibilities in the NASA infrastructure to accomplish this goal. 
- It is essential that the overlap of responsibilities among NASA centers and between centers and headquarters be substantially reduced in order to optimize the influence of the principal investigators and the likelihood of successful experiments.

- Measures should be taken to improve coordination and cooperation between the life sciences administration and the microgravity research administration concerning biological and biotechnological research on cellular and subcellular processes and mechanisms. The strong scientific coupling between biotechnology and other microgravity disciplines through the fluids and transport theme should be recognized in any administrative reorganization.

- All data, including acceleration measurements, should be made available immediately to the principal investigator. Current delays in providing data extend many months beyond the flights. NASA must coordinate the operations of various offices so that priority is given to the processing of experimental data.

- It is necessary to increase substantially the number of ground-based investigations to ensure the future supply of high-quality flight experiments. Consequently, the budget for ground-based research should be increased as a fraction of the entire microgravity program. To the maximum extent feasible. funding for ground-based research in the microgravity program should be protected from temporary budgetary fluctuations.

- In addition to its role in developing flight experiments, the ground-based program can provide important scientific and technical data for other purposes. A ground-based study can therefore be judged successful even if a flight experiment does not result.

- Microgravity research is much broader than the topic of materials processing in space and should be identified as microgravity research in all official documents, including the federal budget. Microgravity research better describes the activity and its actual and potential accomplishments. Materials processing in space characterizes only a fraction of the activity and promotes a misleading impression of the potential benefits and scope of the program.

- The microgravity sciences and applications program and the commercial development program have a large area of overlap and common interest. Coordination between these programs and an equally stringent review process for each should be fostered by NASA for their mutual benefit.

- NASA should establish mechanisms for continuous, unrestricted submission of research proposals in order to optimize quality and take advantage of scientific advances. Unrestricted submission of research proposals would parallel approaches of other funding agencies and would enable continual scientific advance.

- To manage the diverse disciplines in microgravity research, it is necessary to increase the breadth and experience of the scientific staff at NASA headquarters. A rotation of prominent scientists on leave from universities, national laboratories, and industry is one mechanism to be explored. Introduction of active scientists in the administration of the program would be highly beneficial. 
- Prompt documentation of experimental results should be required and enforced. Reports of all experiments, including unsuccessful efforts, should be accessible to all interested parties. A concern is that some investigators might not report results because they are proprietary or inconclusive. The lack of an available report could lead to unnecessary duplication of efforts.

- NASA should organize and maintain an accessible archive of microgravity research results. This archive should contain a bibliography of all published scientific papers and reports on microgravity subjects and should preserve the original spaceflight data sets, such as photographs and electronically recorded data.

An additional point of concern is that given the long time scale for the development through flight of a space experiment, there is a real danger that the scientific goals of the experiment might be bypassed by new developments or by major shifts in the value ascribed to the work. There is also the possibility that the principal investigator may lose contact with the field. Several of the above recommendations may be useful in this regard. Anything that NASA can do to shorten this time frame would be beneficial. This topic is explored in more detail in Chapter 8 , which also contains some additional scientific and administrative recommendations specific to the flight program.

\section{REFERENCE}

1. Space Studies Board, National Research Council. 1992. Toward a Microgravity Research Strategy. National Academy Press, Washington. D.C. 


\section{PART II}

\section{Scientific Issues}





\section{Fluid Mechanics and Transport Phenomena}

\section{INTRODUCTION}

The field of fluid mechanics and transport phenomena plays a dual role in the microgravity research program. It stands as a distinct disciplinary area but also appears as a theme running through other microgravity disciplines. Presentations in other chapters of this report reflect the role of the fluids and transport discipline as providing supportive science for other microgravity disciplines.

Many fluid mechanics and transport phenomena (heat and mass transfer) are influenced directly by gravity. Such phenomena are also vital elements in a wide range of physical, chemical, and biological systems, many of which are important in both terrestrial- and space-based technologies. The main characteristics of a low-gravity environment in relation to fluid mechanics and transport phenomena are the diminished importance of buoyancy-driven flows and sedimentation, and the relative increase in importance of other forces, such as surface tension at fluid-fluid interfaces. These effects have important consequences not only for the conduct of research in other microgravity disciplines but also in the development of many mission-enabling technologies. The fluid mechanics and transport phenomena area of the microgravity research program should have both fundamental and applied objectives:

- Fundamental problems. A key objective for fundamentally oriented research should be the identification and description of new phenomena that may influence transport and other applications in microgravity due to the change in parameter range from the ground-based norm. ${ }^{1}$ A second general objective for fundamental research should be to focus on problems in which a low-gravity

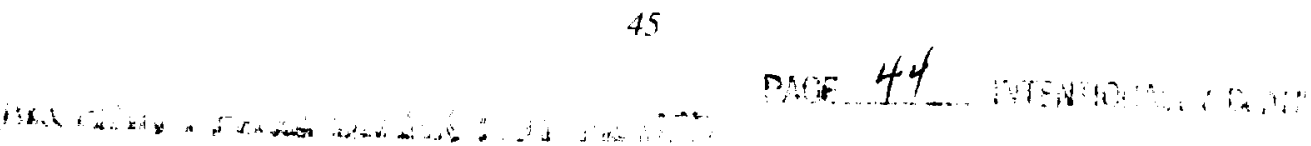


environment can contribute to the fundamental understanding of observed phenomena by providing a unique experimental window.

- Applications. Transport problems are relevant to the other disciplines in the microgravity program and to mission-enabling technologies, including, for example, understanding how altered transport phenomena in microgravity influence the operation or adaptation of biological systems to space, understanding how combustion processes change, or understanding how materials processing is modified.? Many mission-enabling technologies involve transport phenomena, but predictive models for low-gravity performance and operation are frequently inadequate. A knowledge base should be established for the efficient design, development, and operation of such technologies.

\section{ST ATUS}

A few crude demonstrations, consisting primarily of the unusual effects of surface tension on fluid behavior in microgravity. were performed aboard Skylab. ${ }^{3}$ These represented the first fluid experiments in a low-gravity environment. It should be noted that despite the completion of more than 50 space shuttle flights to date, relatively little time was allotted to microgravity research on fluid dynamics and transport phenomena. Six fluid experiments by European scientists were performed in 1983 aboard Spacelab-1. Five of those concerned the effects of capillarity on fluids: isothermal meniscus between two plates of unequal radii; free surface oscillations in drops; liquid-column stability with rotation, vibration, and stretching; axisymmetric free surface behavior due to forced disturbances in partly filled containers; and kinetics of the spreading of a tethered drop. The sixth experiment was designed to study themocapillary flows in a liquid bridge.

Some of these experiments were repeated on the D-1 mission in 1985, along with seven other experiments-two dealing with thermocapillary flows in rectangular containers; two with thermocapillary migration of bubbles and drops; one with diffusocapillary convection in a rectangular container; and two with separation of fluid phases (one in a liquid column and one with bubbles in a reacting liquid).

The first U.S. fluid experiments were performed aboard Spacelab-3 in 1985. One was a geophysical fluid flow cell (i.e., a rotating hemispherical shell of fluid with an electric field to simulate radial gravity forces) and another was concerned with the dynamics of rotating and oscillating drops in acoustic fields. The third, which was not primarily a fluid experiment, studied the growth from solution of triglycine sulfate crystals by means of holograms and indicated the reduction, during crystal growth, of buoyancy-driven convection. It was repeated on the IML-I mission in 1992.

The evaluation of Spacelab-1 fluid experiments noted a number of prob- 
lems, including power limitations, limitations on time available for experiments, an insufficient theoretical basis, shortcomings in equipment performance, and the poor quality of data transfer to the ground. ${ }^{4}$ Reviews of the experimental results of the D-1 mission" also indicated numerous problems in equipment performance, high $g$-jitter accelerations, and diagnostics. Thus, many of the results were essentially qualitative and descriptive.

The USML-I mission, which was launched in June 1992, was the first microgravity flight dedicated solely to U.S. scientists. Thirty-one investigations were included in the payload, with two of the three major facilities for fluid research. The first, the Surface Tension-Driven Convection Experiment (STDCE), was dedicated to a single investigator; the second, the Drop Physics Module (DPM), involved three investigators. A Space Acceleration Measurement System (SAMS) was also included to enable the acceleration environment aboard the shuttle to be described accurately.

The STDCE $E^{6-14}$ conducted in an interactive mode on the USML-1 mission was the first thermocapillary flow experiment with state-of-the-art diagnostic techniques to yield quantitative flow field and temperature data over a wide range of conditions with two different heating modes and various initial interface shapes, which are unique to the microgravity environment. The nature and extent of large Marangoni number steady thermocapillary flows in a cylindrical chamber were determined. The $g$-jitter due to thruster firings and other spacecraft activities did not affect the experiments. No flow oscillations were observed even for Marangoni numbers larger than 10, which indicates that the Marangoni number alone is not sufficient to specify the onset of oscillations.

The STDCE was designed to determine quantitatively the nature and extent of flows (velocity and temperature distributions) driven in a cylindrical container by temperature gradients along the liquid free surface imposed by different thermal signatures due both to various $\mathrm{CO}_{2}$ laser heat fluxes and to various temperature differences between a heated center post and the cooled wall.

The behavior of drops and shells subject to rotational torques by acoustical fields was studied in the DPM. Another experiment involved determining the surface properties of liquid drops in the presence of surfactants and also the coalescence of droplets with surfactants. The third investigation in the DPM measured the liquid-liquid interfacial tension of a compound drop.

A glovebox facility was also aboard that mission. Thirteen qualitative experiments that were developed relatively inexpensively in a short period of time were accommodated in this way. Six of these were related to fluid research: two were on thermocapillary flows (with one generalizing the results of the STDCE), three dealt with capillarity (with two related to the DPM experiments), and one was concerned with the aggregation of fine particles in air. All of the detailed data have not, as yet, been completely analyzed by the investigators, but the results reported ${ }^{15}$ are interesting and encouraging. 


\section{RESEARCH AREAS}

Priority should be given to the study of phenomena that are unique to the low-gravity environment and to those that are critical to space mission-enabling technologies.

\section{Fundamental Research}

Among the basic topics that may be studied with special advantage in the low-gravity environment are the following:

- Surface tension gradient-driven flow's and capillary effects. The effects of flow phenomena driven by surface tension forces are frequently masked by competing gravitational forces in the terrestrial environment but become significant or dominant in reduced gravity. This provides an opportunity for a unique experimental window that can be used to study surface tension-driven convection phenomena, as well as the microscale physics of the interfacial zone (e.g., surface or interfacial rheology in systems with surfactants or the dynamics of the contact line on a solid boundary), all of which remain poorly understood. Fluid interfaces with surface tension variations are also common for many spaceflightenabling technologies (e.g., boiling, heat transfer, combustion, welding, brazing, or soldering). Processes that rely on gravity-driven separations in the terrestrial environment, such as the removal of gas bubbles from a liquid, must be accomplished by other means in microgravity environments (e.g., by thermocapillarydriven migration via nonuniform temperature fields). Frequently, however, additional study will be necessary to enable practical implementation of these processes.

- Multiphase flow's. Many important phenomena involve multiphase flows or two-fluid systems (e.g., bubbles, drops). In ground-based studies, gravity imposes a specific orientation on multiphase fluids, structures, and phase organizations (e.g., gas-liquid, liquid-solid). Furthermore, the systems are generally too complex for fundamental predictions and simulations. Thus, gravity cannot be separated from the observed phenomena in ground-based studies. In a reduced-gravity environment, the gravitational phase orientation is greatly diminished, and it is expected that multiphase flows and associated transport phenomena will become significantly altered. Low-gravity experiments might not only contribute a foundation for space-enabling technologies, as discussed below, but also contribute to an understanding of the essential physics of terrestrial multiphase flows.

- Diffusive transport processes. Under normal gravitational conditions, multicomponent fluids experience various modes of thermosolutal convection (depending on the relative orientations of temperalure and concentration gradients with each other and with the gravity vector). In addition, there are other

effects due to important convection phenomena that derive from the differences 
in diffusivities for heat and mass. Such phenomena occur in many materials and industrial processes (e.g., macrosegregation). With reduced-buoyancy convection, there is a significant opportunity to obtain a better fundamental understanding of other complex temperature- and concentration gradient-induced interactions, such as Soret and Dufour effects, which are usually overshadowed under terrestrial conditions.

- Colloidal phenomena. Colloidal suspensions are representative of a larger class of systems in which surface forces play a critical role. In many instances, however, understanding the consequences of these forces is complicated by competing gravitational effects. Although gravitational effects can be minimized in the terrestrial environment by density matching, this frequently may change the chemical environment or require introduction of materials different from those of direct interest. Thus, the microgravity environment provides an opportunity for experimental studies of phenomena such as order-disorder transitions in dispersions of hard spheres or electrohydrodynamic effects such as drop deformation in electric fields, which can strengthen the fundamental understanding of those physicochemical interactions and their macroscopic manifestations in complex fluids.

The above topics are also important to biotechnology, combustion, and materials science. Some of them are discussed in subsequent sections that deal in detail with those areas. Beyond these specific discipline-based areas, microgravity offers the possibility for experimental studies of fundamental fluid physics phenomena that, in the terrestrial environment, cannot be done or can be done only for limited parameter ranges. One example that has received significant study already is the dynamics of individual bubbles or drops in acoustic fields.

\section{Technology Development}

One of NASA's missions is to "provide technology for present and future civil space missions and provide a base of research and technology capabilities to serve all National space goals."16 Many of the technologies required for NASA programs involve transport phenomena, including power systems; thermal management systems; spacecraft fire hazard management; cryogenic engines; fluid systems tankage; physical and chemical life-support systems; and user-support subsystems such as refrigerators. More specifically, multiphase flow phenomena, which are highly gravity dependent, are central to heat and mass transfer in all systems, and gas-liquid contacting for air purification and energy generation also have a major role in chemical processes such as catalysis and ore beneficiation techniques. Fundamental studies of such phenomena will contribute to better process design for the microgravity environment.

Safe and efficient heat transfer design for reduced-gravity applications is 
currently not possible because fundamental knowledge of convection processes at very low Reynolds number, and of boiling and condensation in a low-gravity environment, is lacking.

NASA's long-term mission to explore the universe is also clearly dependent on exploiting the behavior of materials and processes in low gravity. For example, the mining, winning, and processing of extraterrestrial materials will be essential for extensive exploration of the solar system. Since it would be costly to transport Earth-based materials to the Moon and other planets, in situ materials must be considered. Current processing techniques will not suffice for obtaining materials under the unusual conditions encountered on other, nonterrestrial, bodies. Technologies to enable processes such as fluidized-bed hydrogenation, electrowinning, and vapor-phase pyrolysis will eventually be needed, and all involve transport phenomena that are affected by gravity. The technologies for life- and operations-support systems, such as power generation and storage, water purification, oxygen production, and fuel and fluid storage and management, also involve gravity-dependent transport phenomena.

Thus, if reliable, cost-effective, and efficient technologies are to be developed to enable NASA's mission to be successful, the fundamental understanding of the effects of gravity on many transport phenomena is essential. The committee believes that the following topics, in addition to being of intrinsic scientific interest, are critical to provide the knowledge base required to design effective and reliable space-based systems and facilities in which fluid processes are involved:

- Convective processes at low Reynolds number. Buoyancy affects the transport of heat and mass when the Reynolds number becomes small enough in the terrestrial flow of fluids with density variations. Investigation of low Reynolds number flows with density variations, at reduced gravity levels, can reveal the altered nature of the transport phenomena.

- Transport processes with a phase transition. Aspects of condensation, evaporation, and boiling are influenced by both gravity and interfacial forces. How such phenomena differ in a low-gravity environment, with interfacial forces dominant, requires further study.

- Complex materials. The structure and transport of complex materials are often influenced by gravitational effects; for example, multiphase flows in porous media, flows of powders and granular materials, the motion and stability of foams, and the sedimentation of colloidal dispersions. Research is necessary to understand the behavior of such materials in a microgravity environment. This research may also lead to a better understanding of the behavior of complex materials at $1 \mathrm{~g}$.

- Materials processing. Buoyancy, sedimentation, and interfacial phenomena influence such important processing methods as fluidized-bed hydrogena- 
tion, electrolysis and electrowinning, and vapor-phase pyrolysis. Their features in microgravity should be investigated.

- Physical processes in life-and operating-support systems. Some of the effects indicated above apply to such processes as power generation and storage, water purification, oxygen production, and fuel and fluid storage and management.

\section{SUMMARY}

The modifications in fluid flows and in heat and mass transfer processes that occur in the transition between the terrestrial environment and microgravity underlie modifications that occur in many biological processes, materials behavior, combustion, and a number of mission-enabling technologies. These changes result from the diminished significance of buoyancy for gravity-driven motions and the relatively greater importance of other forces. The microgravity environment also provides unique conditions for experimental studies that can contribute new understanding of fluid flow and transport processes that are significant primarily to Earth-based applications.

For these reasons, a research program on fluid physics, aimed at primarily fundamental studies of fluid mechanics and transport phenomena that are partly or completely masked at $I \mathrm{~g}$, has been under way for the past several decades, and the committee recommends that this program be continued. Among the topics that this program should cover are surface tension-driven flows and capillary effects; multiphase heat transfer and fluid flows; and the behavior of complex fluids such as colloids, granular media, and foams, whose dynamical behavior can be strongly influenced by gravity. In addition, some emphasis should be placed on microgravity transport processes that will play a role in the design and operation of future space facilities, such as low Reynolds number convection, boiling, evaporation or condensation, or methodologies that may be required for materials processing, such as fluidized-bed hydrogenation, electrowinning, or vapor-phase pyrolysis.

It is further recommended that steps be taken to enhance interaction between the research community and the engineering community that will design, build, and operate future space facilities to help prioritize studies related to "enabling technologies." Similarly, steps should be taken to foster interdisciplinary research involving fluid dynamics and transport processes in other areas such as the life sciences, materials processing, and combustion.

\section{REFERENCES}

1. Ostrach, S. 1982. Low-gravity fluid flows. Ann. Rev. Fluid Mech., 14:313-345.

2. Ostrach, S. 1988. Industrial processes influenced by gravity. NASA Contractor Report 182140 . 
3. Ostrach, S. 1974. Skylab science demonstrations. Proceedings of the FSRO Symposium on Processing and Manufacturing in Space.

4. European Space Agency. 1985. Assessment of the Results of Fluid Science and Materials Science Experiments Conducted During the Spacelab-1 Mission.

5. Natur Wissenschaften. 1986. D-l Erste Ergebnisse der deutschen Spacelab Mission. Vol. 7, June.

6. Ostrach, S., Y. Kamotani, and A. Pline. 1993. USML-1 Surface Tension Driven Convection Experiment: Preliminary analysis of the data. In International Symposium on Microgravity Science and Applications, Beijing, China.

7. Kamotani, Y. 1993. Results from the Surface Tension Driven Convection Experiment. Presented at Gordon Research Conference.

8. Pline, A., T. Jacobson, Y, Kamotani, and S. Ostrach. 1993. Surface Tension Driven Convection Experiment. AIAA Paper 93-4312. Presented at AIAA Space Programs and Technologies Conference, Huntsville, Ala.

9. Kamotani, Y., S. Ostrach, and A. Pline. 1993. A thermocapillary convection experiment. 1993 ASME Winter Annual Meeting, New Orleans, La.

10. Kamotani, Y., S. Ostrach, and A. Pline. 1994a. Some results from the Surface Tension Driven Convection Experiment aboard USML-1 Spacelab. 1994 AIAA Aerospace Sciences Meeting, Reno, Nev.

11. Kamotani, Y., S. Ostrach, and A. Pline. 1994b. Some velocity field results from the Thermocapillary Flow Experiment aboard USML-1 Spacelab. 1994 COSPAR Meeting. Hamburg, Germany.

12. Kamotani, Y., S. Ostrach, and A. Pline. 1994c. Analysis of velocity data taken in Surface Tension Driven Convection Experiment in microgravity. Physics of Fluids, 6:11.

13. Kamotani, Y., S. Ostrach, and A. Pline. 1994d. Summary of results from the Surface Tension Driven Convection Experiment in microgravity. 45th Intemational Astronautical Congress, Jerusalem, Israel, October 9-14.

14. Kamotani, Y.. S. Ostrach, and A. Pline. 1995. A thermocapillary flow experiment in microgravity. Journal of Heat Transfer, in press

15. Ramachandran. N. 1994. Joint Launch + One Year Science Review of USML-1 and USMP-1 with the Microgravily Measurement Group. NASA Conference Publication 3272.

16. Space Systems and Technology Advisory Committee. 1991. Advanced Technology for America's Future in Space: A Review of NASA's Integrated Technology Plan for the Civil Space Program. Preliminary draft. 


\section{4 \\ Combustion}

\section{INTRODUCTION AND OVERALL GOALS}

The science and engineering of combustion processes involve many disciplines of physics and chemistry. Combustion is a complex process and one that is influenced by gravity in many ways. Gravitational forces are instrumental in the burning of a candle, where buoyant forces lift the hot gases of combustion and allow the convection of fresh oxygen into the flame region. As illustrated below, however, in the scale-up of a combustion experiment for observational purposes, gravity often obscures the phenomenon of interest by introducing buoyancy that is absent in the experiment at the original scale. As much as the atmosphere has been an impediment to optical astronomy on Earth, so has gravity been an impediment to understanding combustion in certain cases. The near absence of gravity in spaceflight situations ensures different combustion behavior compared to that on Earth. Not only would experimentation under microgravity conditions be useful in improving scientific understanding of that behavior, but it is also imperative from the standpoint of spacecraft fire safety.

The uniqueness of the microgravity environment includes a reduction of buoyancy forces, an inhibition of particle or droplet settling, and in some cases, a reduction of dimensionality (e.g., droplet burning tends to become spherically symmetric). Some surmountable challenges do arise, such as the emergence of surface tension as a major mechanism in certain two-phase experiments and the need to design compact optical diagnostic equipment.

The great reduction of buoyancy forces in the microgravity environment allows the study of certain phenomena with much higher resolution because 
length scales can be increased. For example, similitude with the Reynolds number or the Peclet number for turbulent flows can be maintained in microgravity conditions by increasing the length scale and appropriately decreasing the velocity scale. At Earth gravity, such a change would result in a large increase in the Grashof number (a nondimensional magnitude of buoyancy). That is, buoyancy might be insignificant in the phenomenon of original interest but could inadvertently be introduced in the attempt to improve resolution. Under microgravity conditions, however, the magnitude of the Grashof number remains insignificant. The other type of experiment is one in which buoyancy plays a role in the phenomenon of original interest at Earth gravity. Here, the microgravity environment presents an opportunity to treat gravity as a variable input parameter by designing an experiment to be operated in both microgravity and Earth-gravity environments. In this context, it is noteworthy that conduct of the experiment again in partial-gravity environments (Moon, Mars, or the space station centrifuge) could be helpful.

There are a number of important issues in combustion, including flammability, stability, ignition, extinction, flame speed and spread rate, radiation, multiphase effects, critical point behavior, and smoldering. These are all affected by gravity in any experiment on Earth with normal gravity $(1 \xi)$. A further complication is the wide disparity of relevant characteristic times in various subprocesses in combustion. For example, chemical kinetic times can be as short as $10^{-6} \mathrm{~s}$, droplet burning times can be of the order of milliseconds to seconds, and smoldering times can be of the order of minutes to hours. Thus, both time of observation and gravity are variables that must be taken into account in experimentation. This is discussed below under facilities requirements.

The overall goal of a microgravity program in combustion should be to enhance the science base of combustion phenomena where gravity is an important parameter in the problem. Secondarily, the science base should be expanded in areas where meaningful experiments cannot be performed on Earth because gravity interferes with scaled-up experiments. (See Plates 4.1 and 4.2 for examples of low-gravity combustion experiments.)

\section{PROBLEMS UNIQUE TO COMBUSTION}

There have been several recent reviews of the status of research and technology of gravitational effects on combustion. ${ }^{1-3}$ The problem that $1 g$ poses in studies of combustion is best elucidated by considering some dimensionless groupings that naturally arise. One of these is the Grashof number, which indicates the ratio of buoyant to viscous transport. Since density differences of nine to one (cold to hot) easily occur in combustion, gravity acts differently on hot gases than on cold gases. This extreme density difference is common in combustion. In situations at $1 g$ where the size scales of interest are less than the order of $100 \mu \mathrm{m}$ (droplet burning, for example), buoyancy effects are negligible 


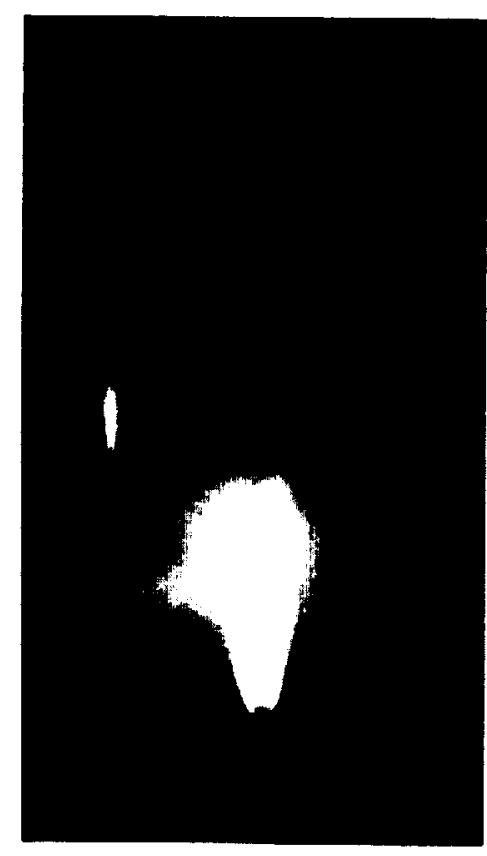

Normal gravity, minimum flame height

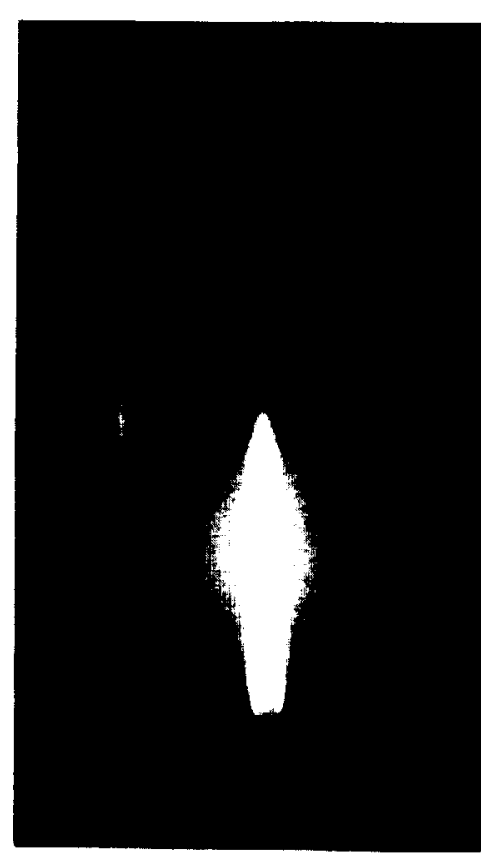

Normal gravity, maximum flame height

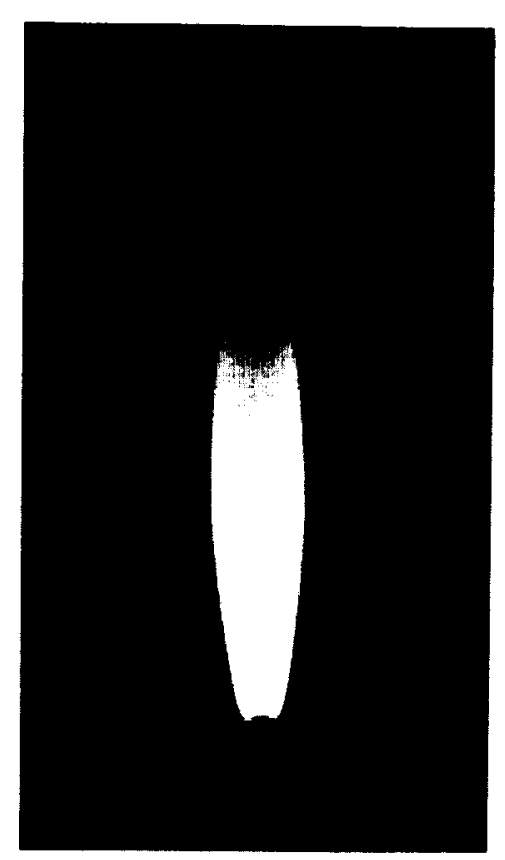

Microgravity
PLATE 4.1 Combustion of a gas-jet (propane) flame in a quiescent atmosphere of air under normal gravity and microgravity conditions (the latter in a drop-tower test at NASA's Lewis Research Center). Due to the buoyancy effects under normal gravity conditions, the flame flickers, leading to the range of flame heights shown in the first two frames. Under microgravity conditions, the flame is very steady and, as may be seen. is considerably broader than under normal gravity conditions. 


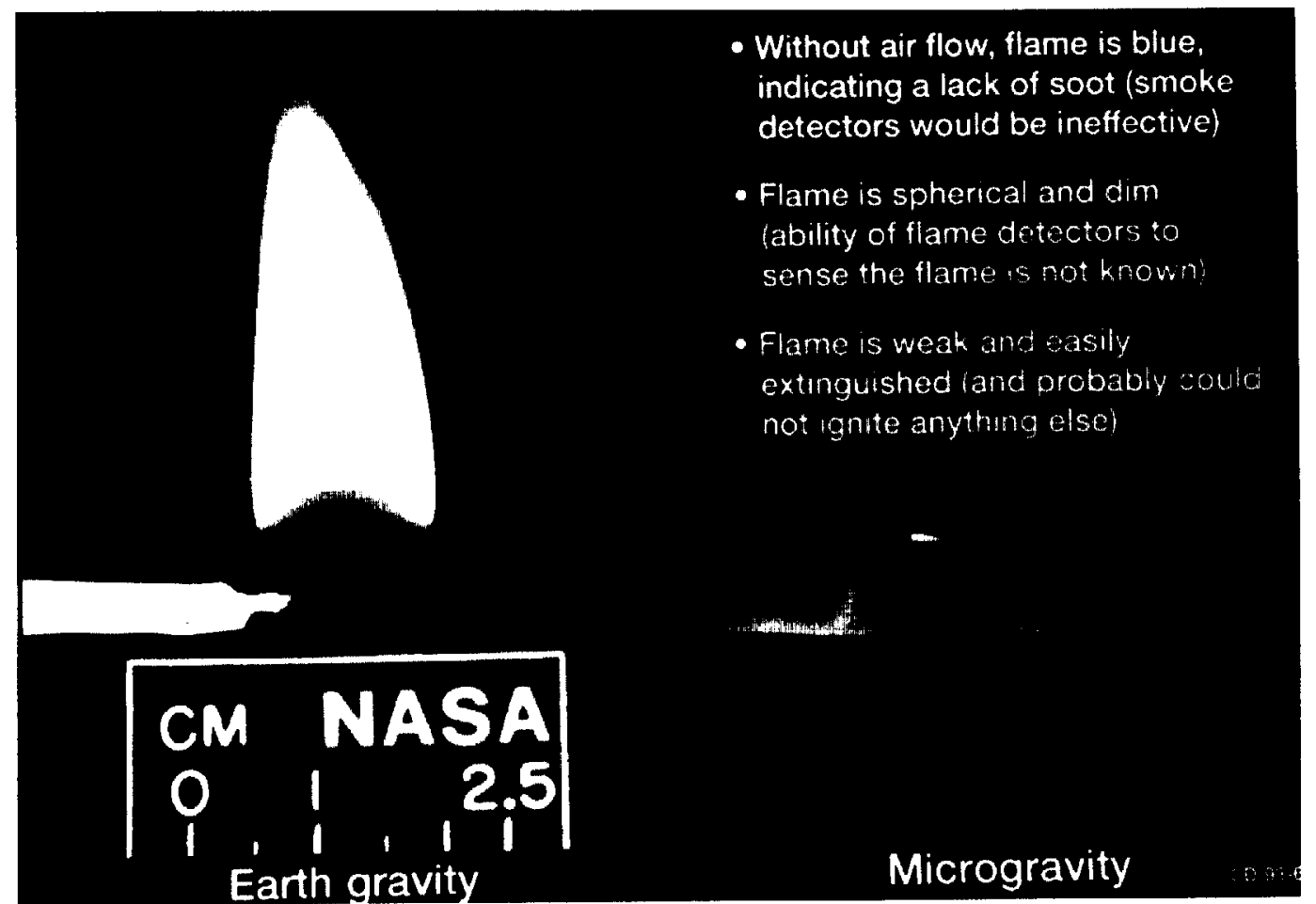

PLATE 4.2 Candle flames. As this figure illustrates, there are dramatic differences between candle burning under normal gravity and microgravity conditions due to the absence of natural convection (buoyancy-induced) in the microgravity case. Under normal gravity, the candle flame is very orange and extends strongly in the vertical

direction due to these convection effects; in the absence of gravity (in a glovebox experiment on USML-1), all transport of oxygen into the flame occurs by relatively slow. very symmetric diffusion processes. resulting in a weak (as indicated by the blueness of the flame), spherically symmetric flame. 
compared to those of molecular transport. These small scales, however, present severe problems in measurement and experiment design. Note that the Grashof number is proportional to the cube of the length scale and is therefore extremely sensitive to experimental size.

If forced convection is present, the appropriate dimensionless group is the Richardson number, which indicates the relative effect of buoyancy-induced velocity to the forced velocity. In situations where a laminar burning velocity of a premixed mixture is the appropriate velocity, the buoyancy-induced velocity can be neglected only for laminar speeds above about $1 \mathrm{~m} / \mathrm{s}$. This is near the upper limit of observed flame speeds for any mixture and is orders of magnitude greater than flame speeds observed near flammability limits, with these limits intensely relevant to the spacecraft fire issue.

In nonpremixed flames (e.g., a jet flame), the Reynolds number based on forced convection velocity must be large compared to the square root of the Grashof number so that buoyant velocities are negligible relative to forced convection velocities. With flow speeds of the order of meters per second or more and size scales of the order of centimeters, this condition on the Reynolds number is met easily. It is not possible to meet this condition for small Reynolds numbers $(<1)$ and length scales of the order of a millimeter or greater at Earth gravity. Small Reynolds numbers at a reasonable laboratory size scale introduce gravitational effects on Earth. The implication is that investigation of the highly important Stokes regime (Reynolds number less than 1 ) is excluded from observation at $1 \mathrm{~g}$. This regime can be of interest, for example, in droplet burning.

In turbulent flows, characterized by high Reynolds number, there can be a large range of size scales from the large eddies to the smallest structures. For buoyancy-induced stresses to be small compared to the turbulent stresses under investigation, flow velocities must be large $(>50 \mathrm{~m} / \mathrm{s})$ in a reasonably sized apparatus (say $1 \mathrm{~cm}$ ). While this may be achieved locally, there are always lowspeed regions in a turbulent combustion experiment for which the buoyancy-free condition will be violated. On the other hand, in practical devices, buoyancy is not present at the speeds, size scales, and geometries of interest. The turbulence prediction problem is difficult enough without gravitational effects, and the complications presented by gravity obscure comparison of theory and experiment.

At the University of Sydney, Sandia Laboratories, and the General Electric Company, turbulent jet diffusion flame studies at $1 \mathrm{~g}$ show clear buoyancy effects that either destroy symmetry or induce axial disturbances that make agrecment between theory and experiment difficult far enough downstream in the flame. ${ }^{4}$ The downstream region is, however, one of the important flame regions for the study of turbulence decay. A database is needed in this region to study closure methods or computational methods for turbulence prediction. As another example, consider droplet burning, a concern in all spray combustion devices. It is usually true that buoyancy forces are small in practical spray combustion devices. However, the droplets are usually small (of the order of $100 \mu \mathrm{m}$ or less) 
in a real combustion chamber, and experimental access on this scale is impossible at the current time. Scaling up the experimental size to the order of several millimeters or centimeters allows experimental access, but then the buoyancy problem appears and the experimental data are not relevant to the real industrial process.

While $1 g$ often presents a hindrance to gaining scientific understanding of combustion phenomena, experiments in the microgravity environment can present challenges in the form of new phenomena that become dominant. These challenges can be viewed as opportunities to address new scientific issues in combustion through microgravity experiments and supporting theory.

Although some problem areas relate to the gravity level, as discussed above, other problems can arise in microgravity experiments of combustion. For example, the fuel/oxidizer ratio, relevant to the issue of spacecraft fire safety, is another variable that must be investigated. The oxygen content of spacecraft atmospheres can vary over a reasonable range and still be satisfactory to humans. Consequently, data for oxygen at only $21 \%$ by volume, common to the $1-g$ problem, may be unsatisfactory. Another problem concerns the ability to acquire enough data for statistical confidence in results. The data acquired in a typical microgravity experiment to date consist of data at one point. This problem is compounded in combustion because of the large number of variables, including the gravity level. Exhaust gas presents yet another problem area in microgravity testing of combustion. Because the exhaust of combustion products is restricted on spacecraft, special means must be employed in experiment design.

Experimental requirements are especially severe in combustion because of the many variables of interest: pressure, temperature, velocity, concentration of major and minor species, and radiation (in the visible, ultraviolet, and infrared). This taxes the field of experimental diagnostics to the limits of technology, especially in view of the compactness requirements of equipment in flight configurations.

\section{STATUS OF GRAVITATIONAL-BASED CURRENT KNOWLEDGE}

The flammability limits of premixed gases are crucial to spacecraft fires and have already been shown to be wider at microgravity than at 1 g.5.6 Both magnitude and orientation of gravity affect flammability limits. The mechanisms operating at flammability limits are currently uncertain; it is not known whether heat loss, chemical kinetics, or various instabilities are the influential phenomena. A series of experiments on flame propagation through gaseous combustible mixtures is needed to evaluate the relative importance of various mechanisms to the flammability limits. The effects of chemical kinetics, radiative heat losses, fluid dynamic strain, mixture ratio, Lewis number, and buoyancy should be determined. The effects of these same parameters on flame front instability and 
cellular flame formation are also of interest. The use of a microgravity environment to separate the buoyancy effect from other potentially important effects would be highly useful for the comparison of theory and experiment and in the practical arena of spacecraft fire safety.

A number of instabilities arise in various gas mixtures that are used to study turbulent flames. The Rayleigh-Taylor ${ }^{7}$ instability requires the presence of accelerations - gravity or otherwise. The density differences that are inherent in flames are the root cause of the Landau-Darrieus instability, ${ }^{8}$ which is not gravitationally dependent. Other instabilities that may depend on gravitational effects are thermodiffusive in nature." The study of flame instability behavior could be carried out fruitfully in microgravity to gain unambiguous information about the causes of instability. Some unique behavior at microgravity has been discovered in the area of self-extinguishing flames ${ }^{10}$ and stationary spherical flames, which involve flame stretch (strain), radiation losses, and various instability mechanisms.

Microgravity studies of gaseous diffusion flames have resulted in the discoveries of new extinction behavior and excessive sooting. ${ }^{11}$ In droplet burning studies, soot problems occur in microgravity in the form of nonremoval of soot by buoyant forces and thermophoretic transport of soot toward the droplet surface. ${ }^{2}$ Because of the elimination of settling effects, important opportunities exist for the study of soot formation and agglomeration. The absence of settling also allows for useful studies of spray combustion and particle cloud combustion.

A primary area of interest in spacecraft fire safety concerns how flame spread over solid and liquid fuels (with and without forced convection environments) is affected by gravity. Buoyancy and surface tension have been shown to be important in ignition and flame spread above liquid fuel pools. Flame spread rates over solid fuels have been found to decrease as the ambient oxygen concentration decreases, and the dependence on the magnitude of gravity increases as the oxygen level decreases. ${ }^{13,14}$ Forced air velocities affect spread rates; spread rates increase with increasing velocity until blow-off occurs. Curious optima on flame spread rate have also been discovered with regard to the convective flow rate in such flames. USML-1 results ${ }^{15}$ indicate that radiation heat transfer is dominant in the flame spread above solid fuels.

Smoldering of solid fuels, often a precursor to fire, will be especially susceptible to gravitation, because of the nonremoval of flammable products of pyrolysis by buoyancy. Furthermore, USML-1 results ${ }^{16}$ indicate that carbon monoxide production from smoldering at reduced gravity is an order of magnitude greater than on Earth. Fire detection and extinguishment methods will also have to be different at microgravity as opposed to those at $1 \mathrm{~g}$. For example. smoke detectors on Earth are located in ceilings because of buoyancy, as are detectors for hydrogen.

Progress is being made in direct numerical simulation of turbulent flames. albeit at fairly low Reynolds numbers (quasi turbulence). These simulations cannot be tested on Earth, however, because of gravitational influences on these 
low-velocity configurations (i.e., because simulation at low Reynolds numbers introduces buoyancy). The same problem exists with large eddy simulations and second-order closure methods (two methods of approximating turbulent transport). The microgravity environment may be useful to verify theoretical methods because flows may be constructed at low velocities and large size scales free of buoyant effects.

\section{SPECIAL INSTRUMENTATION AND FACILITIES REQUIREMENTS}

As mentioned above, the duration of observation is central to the facilities required if combustion experiments at microgravity are to be conducted. NASA facilities include drop towers offering 2 to 5 seconds of test time, airplanes offering tens of seconds in parabolic flight and also allowing various gravity levels, the shuttle offering hours to days, and the future space station with days to years, each with its unique gravity-level capabilities. ${ }^{7}$ Most of the information that has been gained in the facilities used to date has been photographic. It has been found. however, that even in drop tower experiments with a sudden impact at the end, lasers can survive. It appears that more sophisticated interrogation equipment may be employed in the future.

Advanced laser diagnostics ${ }^{18}$ have been used in ground applications to generate much fundamental combustion and aerodynamic data and to study the operational behavior of many kinds of practical devices in a nonperturbing manner. The capabilities in this field are driven by advances in the equipment used: (1) improved laser power, higher repetition rates, smaller physical size, and increased electrical-to-optical efficiency; (2) greater multielement detector sensitivity, faster gating capability, smaller pixel size, and larger formats; (3) faster computer speed, smaller physical size, increased data storage capability, and lower power consumption; (4) more choices in fiber-optic characteristics; and (5) advances in spectrograph designs. Based in part on these advances, spectroscopic diagnostics have progressed from laboratory demonstrations to measurements in full-scale test stands. Hardened, mobile apparatus have been successfully developed that. with further evolution, are suitable for flight applications.

Available laser powers and detector sensitivities now permit many nonintrusive diagnostics to be performed using one- or two-dimensional imaging configurations. The availability of specific wavelengths, especially those in the ultraviolet, has caused dramatic revivals in the importance of some diagnostics (e.g., excimer-pumped spontaneous Raman scattering). Some of the currently used technology derives from related areas: flight-certified lasers (especially solidstate) from satellite communications; intensified detectors and spectrograph designs from satellite, astronomical, and military imaging; and diode laser/fiberoptic technology from communications and photonics. Much recent work has been directed toward instantaneous imaging in short-duration facilities such as 
shock tunnels. Recent advances in diagnostic strategies have permitted measurements to progress from semiquantitative visualization to more quantitative results. These developments have greatly increased the utility of the data obtained for the validation of computational fluid dynamic (CFD) codes.

In the absence of buoyancy, the structures of many microgravity flames are exceedingly fragile; the use of physical probes is unacceptably perturbing. Many of the general improvements in equipment choices described above support current and future microgravity applications. The need to work with hardened, mobile apparatus in short-duration, hostile, test cells has paved the way for the drop tower arena. Flight-certified equipment with reasonable size and power requirements is compatible with reduced-gravity aircraft flights. Space-qualified equipment that has been used in satellites is, of course, especially relevant to the space shuttle.

To be useful in short-duration, reduced-gravity, ground-based experiments, diagnostic approaches must be at least line imaging. Most microgravity combustion-diagnostic work to date has involved either physical probes or simple and relatively conventional backlighted schemes with standard, high-speed, photographic recording. A diagnostic technique as simple as recording luminosity has provided significant insight into flame structure (e.g., flamefront location). Microgravity research should be increasingly impacted by many of the techniques becoming standard in combustion research, such as the direct, digital recording of fluorescence from flame radicals (e.g., planar laser-induced fluorescence, PLIF). Laser sheet illumination will allow resolution of spatial structures not possible with line-of-sight approaches (e.g., schlieren) that use backlighting. Laser light scattering from soot should help characterize soot formation and from added seeds permit velocity field imaging using methods such as particle image velocimetry (PIV). Multiphase flow phenomena should be especially interesting in microgravity, and many of the droplet/spray diagnostics developed over the last decade should be relevant.

\section{RECOMMENDATIONS AND CONCLUSIONS}

In the field of microgravity research on combustion phenomena, there are several important areas. The following conclusions and recommendations are in order of priority:

1. Microgravity research in combustion is needed because of fire concerns on spacecraft and potential lunar and Mars bases. Implicit here is that variable gravity would be helpful.

2. The needed scientific information concerning the problem of fire involves the subfields of ignition, flammability limits, smoldering, extinguishment, and flame spread.

3. Turbulent combustion research is needed because of its importance on 
Earth. Reduced gravity would allow a size scale-up without major buoyancy effects, permitting scientific access to the small scales of turbulence that are important to the problem.

4. Laminar premixed and diffusion flames and spray-flow interactions are important on Earth. Experiments should be performed at reduced gravity to allow scale-up in size without complications due to buoyancy.

Other recommendations concerning equipment and facilities follow:

- An extended orbiting platform capability is important because some combustion phenomena are long-duration events and may require a survey of a wide range of parameters. The demonstration of reproducibility is required and adds to the need for an extended platform.

- The space available on spacecraft for combustion experiments is generally limited, and many different types of measurements are required. For these reasons, the development of miniaturized diagnostic equipment should be undertaken.

\section{REFERENCES}

1. Faeth, G.M. 1991. Homogeneous premixed and nonpremixed flames in microgravity: a review. Pp. 281-293 in Proceedings of AIAA/IKI Microgravity Sciences Symposium, American Institute of Aeronautics and Astronautics, Washington. D.C.

2. Law, C.K. 1990. Combustion in Microgravity: Opportunities. Challenges, and Progress. AlAA Paper No. 90-0120.

3. Strahle, W.C., and S.G. Lekoudis. 1985. Evaluation of Datta on Simple Turbulent Flames. Technical Report, Air Force Office of Scientific Research.

4. Strahle, W.C., and S.G. Lekoudis. 1985. Evaluation of Data on Simple Turbulent Flames. Technical Report, Air Force Office of Scienctific Research.

5. Ronney, P.D., and H.Y. Wachman. 1987. Effect of gravily on laminar premixed gas combustion I: Flammability limits and burning velocities. Combustion and Flame, 62:107.

6. Strehlow. R.A.. K.A. Rox, and B.L. Wherley. 1987. The effect of gravity on premixed flame propagation and extinction in a vertical standard flammability tube. 21st Symposium (International) on Combustion. The Combustion Institute. Pittsburgh.

7. Taylor, G.I. 1950. The instability of liquid surfaces when accelerated in a direction perpendicular to their planes. Proc. R. Soc. London, 201 A:192-196.

8. Landau, L.D., and E.M. Lifshitz. 1959. Fluid Mechanics. Pergamon, London.

9. Williams, F.A. 1985. Combustion Theory, 2nd Ed. Benjamin/C ummings, Menio Park, Calif.

10. Ronney, P.D. 1988. Effect of chemistry and transpont properties on near-limit flames in microgravity. Combust. Sci. Technol., 59:123.

11. Edelman, R.B., and M.Y. Bahadori. 1987. Effects of buoyancy on gas jet diffusion thames, experiment and theory. Acta Astronautica. 13:681.

12. Shaw, B.D., F.L. Dryer, F.A. Williams, and J.B. Haggard. 1988. Sooting and disruption in spherical symmetrical combustion of decane droplets in air. Acta Astronautica. 17:1195.

13. Olson, S.L., P. Ferkul, and J. T'ien. 1989. Near-limit flame spread over a thin solid fuel in microgravity. 22nd Symposium (Intemational) on Combustion. The Combustion Institute, Pittsburgh.

14. Bhattacharjee, S., R.A. Altenkirch, S.L. Olson, and R.G. Sotos. 1988. Heat transfer to a thin solid combustible in flame spreading at microgravity. Eastern States Section of the Combustion Institute. Pittsburgh 
15. Ramachandra, P.A., et al. 1994. The behivior of flames spreading over thin solids in microgravity. Twenty-Fifth Symposium (International) on Combustion. The Combustion Institule. Pittsburgh.

16. Stocker, D.P., S.L. Olson, J.L. Torero, and A.C. Fernandez-Pello. 1993. Microgravity smoldering combustion on the USML-1 Space Shuttle mission. Heat Transfer in Microgravity. Vol. 260. C.T. Avedisian and V.A. Arpaci, eds. ASME Heat Transfer Division.

17. NASA. 1989. Microgravity Combustion Science: A Program Overview. NASA TM 101424.

18. The following four paragraphs were provided by Gregory Dobbs of United Technologies Research Center. 


\section{5 \\ Biological Sciences and Biotechnology}

\section{INTRODUCTION}

Progress in biology research is often a consequence of the investigator's skill in maintaining experimental control through the use of diverse chemical, physical, genetic, and immunological techniques. Major advances in molecular biology and biotechnology are critically dependent on refinements of existing techniques and the development of new and better ones.

Microgravity research in the biological sciences can help to further these advances. It can also provide fundamental knowledge important to NASA's overall goals. To do so, however, a systematic program is needed to identify and explore those cellular and biomolecular processes, mechanisms, structures, and assemblies that are affected by transfer to the microgravity environment. For the several areas of biology and biotechnology, such as cell culture, cell fusion, electrophoretic separation, and protein crystal growth, in which the effects of microgravity have been demonstrated, further research is needed to identify and explore mechanisms by which gravitational effects are realized. New methods and techniques to take advantage of the potential of microgravity applications need to be developed.

\section{JUSTIFICATION FOR MICROGRAVITY RESEARCH}

Gravity affects biological systems through its influence on the transfer of mass and heat, particularly in the area of fluid dynamics and transport, as well as its less well understood impact on cell structure and function. The basis of 
modern research in the biological sciences is the study of molecules, molecular assemblies, organelles, cells, and cell assemblies in controlled fluid and chemical environments. The impetus for microgravity research is that it may lead to new knowledge about biological systems, to improvements in current experimental techniques, and to the development of new experimental approaches to biological problems.

The NASA research program in the biological sciences and biotechnology has focused on three primary areas of biological interest: (1) separation physics aimed at providing improved resolution and sensitivity in preparative and bioanalytical techniques; (2) cell biology, cell function, and cell-cell interactions; and (3) physical chemistry of biological macromolecules and their interactions, including studies of protein crystal growth directed at supporting crystallographic structure determinations. Brief discussions of microgravity research in each of these areas provide an introduction to the work that has been done and to some of the realized or anticipated scientific and technological benefits.

- Separation physics.' Progress in the biological sciences and biotechnology has been limited to some extent by the failure of fluid-based techniques of current separation and analytical methods to achieve the necessary resolution, a failure exacerbated by the effects of gravity. Two such effects are densitydependent thermal convection and sedimentation, both of which perturb processes used to separate and purify proteins, fractionate cell components and organelles, or separate mixtures of naturally or genetically engineered cells.

- Cell biology. ${ }^{2}$ Modern research in the biological sciences and biotechnology relies on the manipulation of cells of living organisms. In the case of biotechnology, the purpose of these manipulations may be to produce useful molecules-both naturally occurring and of artificial origin-in useful quantities; to develop new organisms or new biological molecules for specific uses; or to improve yields of plant and animal products through genetic alteration. Recombinant techniques, for example, make it possible to produce natural or artificially mutated versions of proteins exhibiting a wide range of activities and uses, scientific and medical, in heretofore extraordinary quantities. The techniques essential to these manipulations are applied in aqueous environments and are subject to fluid dynamics and transport processes. Gravitational effects may have important consequences-experimental in the case of scientific research and financial in the case of biotechnological production. Examples include fermentation processes; compartmental targeting of expressed products within the cell; and the ultimate purity, structural integrity, and activity of a protein product. Particle sedimentation under the influence of gravity, for example, can interfere with aggregation processes such as those mediating cell-cell interactions, cell fusion, cell agglutination, and cellular interactions with substrates.

- Molecular structure. ${ }^{3}$ A full understanding of the functions of biological macromolecules, and of the chemical and physical effects that they organize 
and manage to achieve these functions, is not possible without detailed knowledge of their three-dimensional architectures. Nor is it possible to engineer new proteins, whether for medical uses or as complex biomaterials, without an ability to relate molecular structure and function. Protein crystallography, currently the principal method for determining the structure of complex biological molecules, requires relatively large, well-ordered single crystals of useful morphology. Crystals with these qualities may be difficult to produce for a variety of reasons, some of which may be influenced by gravity, through density-driven convection and sedimentation. Protein crystal growth experiments conducted aboard the shuttle have provided persuasive evidence that improvements can, in fact, be realized for a variety of protein samples.

Another justification for microgravity research concerns the importance of biological research to technological developments supporting crewed space missions. A number of ambitious goals for human exploration of the solar system have been advocated. These include a permanently inhabited base on the Moon and a program of crewed missions to Mars.

Experience from a variety of extended missions in Earth orbit has shown that prolonged exposure to microgravity can have profound effects on human physiology and that return to terrestrial gravity may require long periods of readjustment and reconditioning. Research in the life sciences has begun to pinpoint some of the physiological systems most affected by habituation to reduced-gravity conditions. but little is known about the effects of microgravity on the underlying cellular processes. Additional biological science research is required in support of life sciences and human physiology goals if we are to meet the full range of life-support challenges presented by human space exploration. These challenges range from the development of methods for ameliorating the physiological effects of microgravity and the return to terrestrial gravity to the development of technologies for meeting nutritional, respiratory, and waste disposal needs. This research should explore microgravity effects on cells and cell processes, particularly those involving human physiological systems at risk, as well as survey microgravity effects across a wide range of model systems and organisms.

A final justification for microgravity research in the biological sciences is the anticipation that new knowledge will be accumulated by the optimized production of small amounts of microgravity-derived biomaterials such as purified proteins or protein crystals. Such precious specimens may induce the research community to conduct experiments in novel and previously inaccessible areas of inquiry. Microgravity research may also facilitate otherwise labor-intensive, and therefore seldom-attempted, efforts on Earth and make precious reagents and biomaterials accessible to a wider community. It is possible that knowledge gained in microgravity experiments will permit improvements in terrestrial strategies in such areas as bioseparations and materials processing. It is also possible that methods and processes may be discovered that are possible only in space. 


\section{FUNDAMENTAL RESEARCH AREAS}

NASA has for some lime nurtured a program of flight experiments to delineate specific advantages and limitations of microgravity research with regard to biological sciences. Experiments were designed to produce results that were anticipated from model experiments or analyses but could not be tested in terrestrial laboratories. Because of broad interest in these studies, scientists in a wide range of disciplines, in both academia and industry, were encouraged to participate and judge the potential benefits of biology and biotechnology research in microgravity. Typical of innovative science, preliminary experiments in electrophoresis. phase partitioning, protein crystal growth, and cell culture yielded unexpected results. In some cases, additional or unanticipated advantages of a microgravity environment appeared; in other cases, secondary effects matsked in terrestrial experiments became dominant. Research areas in the biological sciences that have demonstrated potential benefit from microgravity experimentation are presented below.

\section{Investigations of Mechanisms of Macromolecular Interactions at the Subcellular Level}

Biotechnology is for the most part applied molecular biology in the sense that molecular events occurring within the cell, or among groups of cells, are altered or manipulated in some way to achieve a desired end. The methods involved may be based on recombinant DNA technology, cell fusion, or a number of other cellular or subcellular approaches. The objectives may be to produce altered cell types in culture, specific immunoglobulins, drugs, commercial enzymes, proteins, other pharmacological agents, and even whole organisms having improved properties. In order to apply molecular biology at the cellular and subcellular level, where microgravity is intended as a probe or a tool, one must know with some assurance exactly which processes and mechanisms are, in fact. affected by gravity. At present, no adequate knowledge base exists.

The underlying premise for performing biology and biotechnology experiments in space, and for the development of molecular biology tools based on the absence of gravity, is that the microgravity environment must have direct effects on cells and subcellular events. A similar premise underlies research to understand the short- and long-term physiological effects of microgravity on humans and to develop methods and procedures to minimize or eliminate any negative impacts of the effect of microgravity. The following questions must be answered: What are the effects of microgravity on cells and subcellular events, what are their magnitudes, and what are the consequences? Even if the consequences are insignificant, the answers are needed in order to understand these systems fully.

A concerted effort should be made to determine convincingly whether microgravity has an observable effect on the growth, development, and structure of 
living cells. Observations and biochemical assays should be conducted on organisms exposed for various periods of time to a microgravity environment to determine if there are any gross changes in character and, if so, to what general categories of events these effects should be ascribed.

Following a careful cataloging of microgravity effects on cells, studies should be extended to more complex cell-cell systems and the study of longer-term trends. These might include, depending on the earlier results, investigations of cell-cell and cell-matrix interactions and how they are affected by microgravity, as well as the transport of materials between cells. Mixed cell systems should be examined to determine if intercellular communication is affected. Other areas for investigation include the secretory and transport properties of cultured endocrine cells, the development of nerve cells, plant cell-wall structural changes, chemotaxis, virus propagation, adhesion processes, and the assembly of organized macromolecular aggregations such as viruses, membranes, and cell organelles.

In the long term, processes such as multigenerational changes, embryogenesis, evolution, and complex cell interactions approaching the organism level must be addressed. This level of sophistication would also include viral, fungal, and bacterial infection of cells and the role that microgravity plays in alterations of these interactions.

\section{Growth of Biological Macromolecular Crystals}

Macromolecular crystallography has proved to be a powerful tool for basic research in biochemistry and molecular biology. As a result, it has attracted the interest and support of the pharmaceutical, chemical, and biotechnology industries, particularly for use in structure-based drug design and protein engineering. Crystals of an appropriate size and quality are a prerequisite for determination of the threedimensional structures of proteins and nucleic acids. The major obstacle in the application of crystallographic methods to proteins and in the application of protein structure in structure-based drug design is growing suitable crystals.

Initial research in crystal growth under microgravity conditions concentrated on the preparation of metals, alloys, and electronic materials and the identification of model systems. Results of these experiments suggested that growth in microgravity might also have beneficial effects on the preparation of protein crystals. Rationales included the elimination of sedimentation, which might create more nearly isotropic or uniform growth conditions and thereby produce improvements in size and shape, and the elimination of density-driven convection, which might create conditions closer to diffusion-limited growth and thereby improve crystalline order and extend $x$-ray scattering limits to higher resolution. An extensive program of experiments in protein crystal growth in microgravity has been conducted in recent years to test these possibilities. This program has been international in scope and has employed a variety of orbital vehicles, but the majority of these experiments have been sponsored by NASA and conducted aboard the space shuttle. 
In recent years, successful macromolecular crystallization experiments have been performed by U.S. investigators using a number of instruments of U.S. and European design. In early Vapor Diffusion Apparatus (VDA) experiments, crystals of $\gamma$-interferon, porcine elastase, and isocitrate lyase grew larger, displayed more uniform morphologies, and yielded diffraction data of higher resolution than equivalent crystals grown on Earth. ${ }^{4}$ Similar results were obtained for canavalin ${ }^{5}$ and positive results continue to accumulate from this apparatus. ${ }^{6}$ On USML-1, experiments in the glovebox, using a modified vapor diffusion technique, yielded crystals of malic enzyme of substantially enhanced properties. 7.8 In the Cryostat device provided by the German Space Agency DARA, crystals were obtained by liquid-liquid diffusion of satellite tobacco mosaic virus (STMV) that were larger and diffacted to a higher resolution than the best obtained in the laboratory. Data from these crystals allowed structure determination of STMV at $1.8-\AA$ resolution, the highest ever achieved for any virus crystal. ${ }^{9-11}$ (on IML- 1 in the Cryostat ${ }^{12}$ and on IML-2 in the European Space Agency (ESA) Advanced Protein Crystallization Facility (APCF), which also supports liquidliquid diffusion experiments, a number of morphological alterations to crystals of canavalin and another larger virus, turnip yellow mosaic virus (TYMV), were clearly demonstrated. A third technique, temperature-induced batch crystallization, has recently produced larger, higher-resolution crystals of insulin. ${ }^{13} \mathrm{~A}$ group of U.S. investigators also carried out protein crystal growth experiments on the Russian Mir, using a number of different crystallization devices. In agreement with the results cited above, they reported that experiments on 5 of 21 proteins produced results superior to those obtained on Earth. ${ }^{14.15}$ An interesting aspect of the results seen so far is that when the same protein is crystallized by a variety of different techniques in microgravity, a range of crystalline samples may be expected. ${ }^{16}$ pointing up the need for multiple flight experiments and optimization. These experiments have provided persuasive evidence that growth in microgravity can produce protein crystals of larger size, better shape, and higher quality than have been obtained on Earth. They also show that benefits from microgravity crystal growth can be crucial to success in protein structure determination. ${ }^{17.18}$ (See Figure 5.1 and Plates 5.1 and 5.2.)

On the other hand, although protein crystal growth experiments in microgravity have yielded a variety of encouraging and successful results, they have not shown that protein crystals uniformly display improved properties when grown in microgravity. A reason for this result might be the mismatch between protein and technique. Experiments testing a variety of proteins in a particular apparatus have been illuminating. These experiments could be expanded to include a broader range of proteins, such as membrane proteins, receptor-ligand complexes, glycoproteins, and other problematic macromolecules, to understand better the limits of a given technique and what might be done to overcome them. It is also important, however, that a broader range of techniques be explored in repetitive microgravity experiments. More experiments that use liquid-liquid 


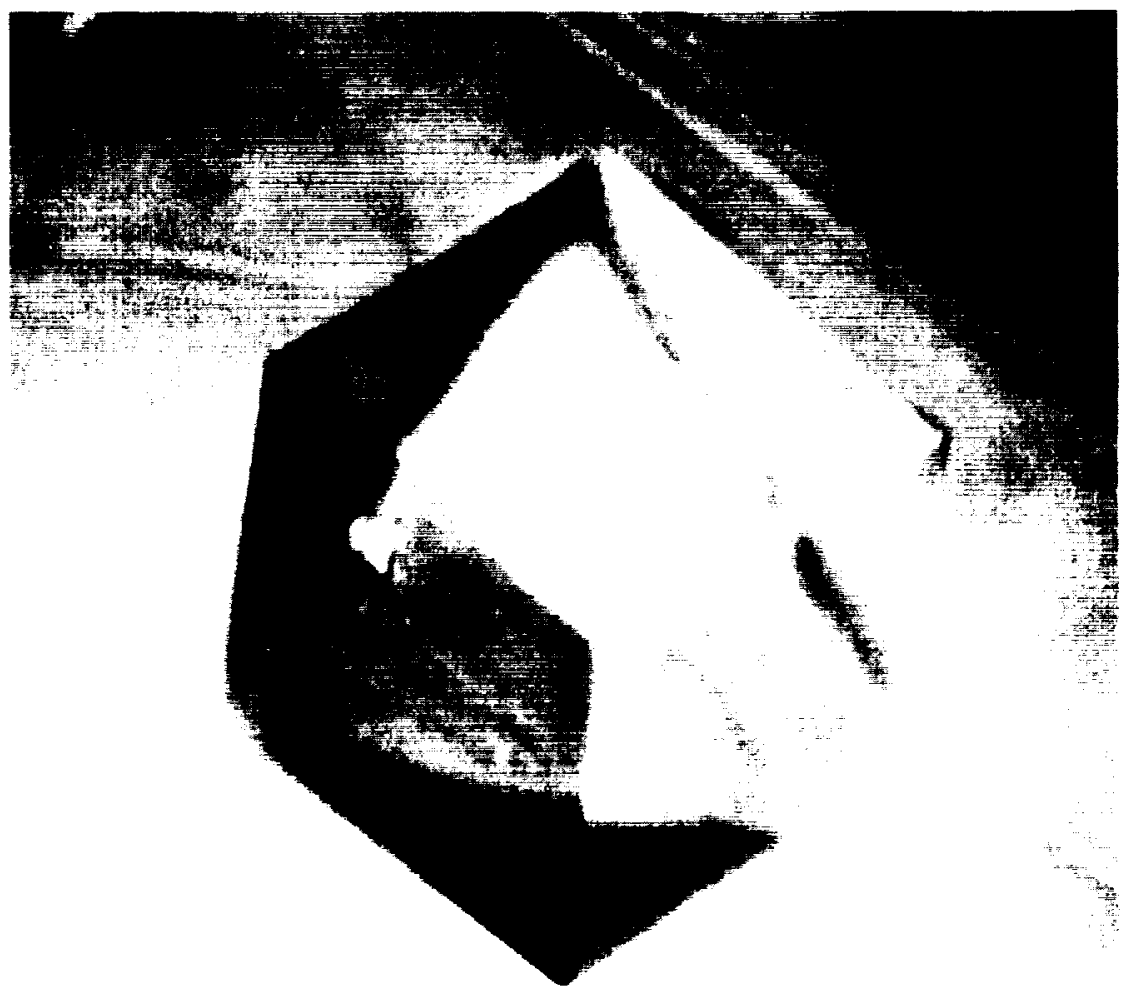

FIGURE 5.1 Crystal of malic enzyme from parasitic nematode Ascaris sutum grown in interactive glovebox experiments aboard the United States Microgravity Laboratory-1 flight in 1992 . While this crystal $(0.20$ to $0.25 \mathrm{~mm}$ on an edge) is about 20 to $25 \%$ of the volume of goot crystals grown on Earth, resolution limits from this crystal exceed by about $0.5 \AA$ the best datal ever collected from crystals grown on Earth.

diffusion or batch methods, for example, will provide a better understanding of the differences among techniques and the results expected to be produced in microgravity. Another reason that not all protein crystals grown in microgravity have shown improved properties might be that crystallization conditions have not been optimized for growth in a microgravity environment. Past results have shown that, more often than not, optimum conditions for growth on Earth are not optimum for growth in microgravity and that the periods of time required to allow crystal growth to go to completion are too short for optimal growth in microgravity. The glovebox experiments in protein crystal growth on USML-1 



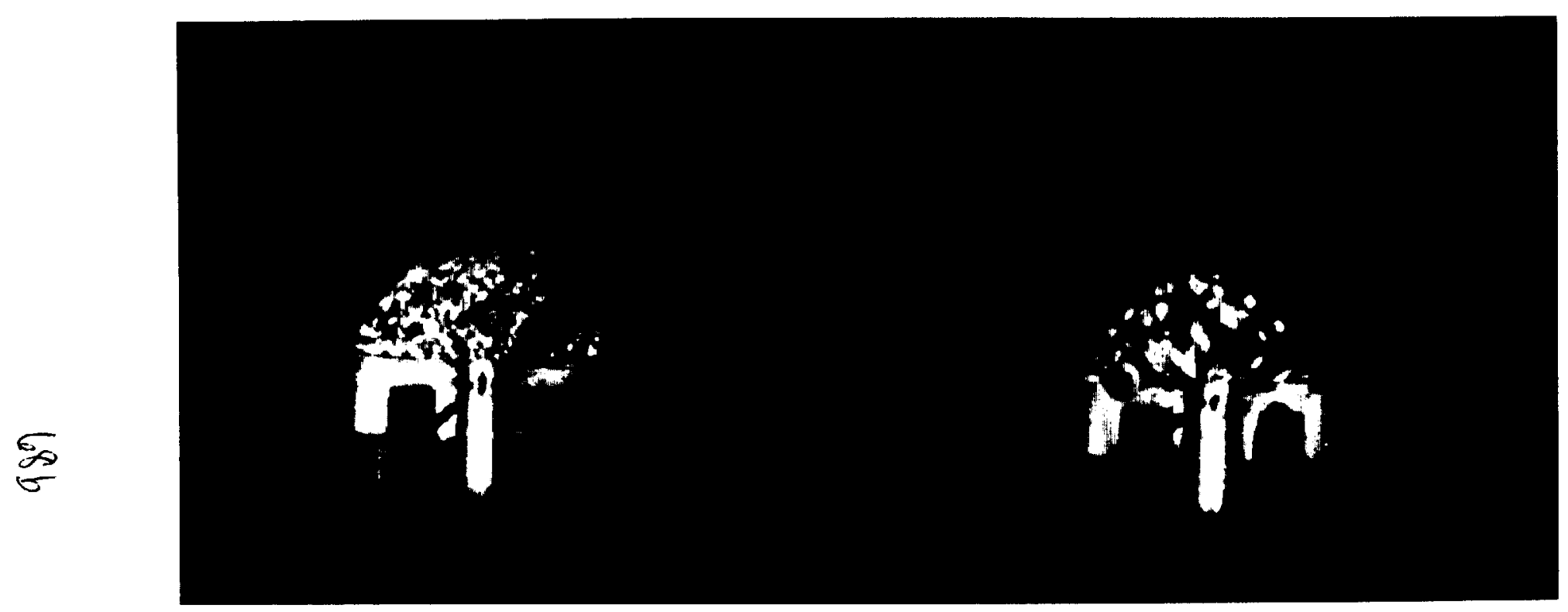

PLATE 5.2 Droplets of protein solution in the Vapor Diffusion Apparatus (VDA) on shuttle mission STS-26. Droplets, about $40 \mu \mathrm{l}$ in size, are poised on double-barreled syringes and are formed by the mixing of protein and precipitant solutions isolated in separate barrels prior to launch. Droplets equilibrate by vapor diffusion against a reservoir of precipitant stabilized in microgravity in a porous high-molecular-weight polyethylene plug (not shown). 
were the first to offer opportunities for iterative experiments in microgravily with an experienced specialist to interpret results and design subsequent experiments. The results of these experiments indicated that iteration can increase the production of crystals of high quality in microgravity. ${ }^{19.20}$

A goal of future experiments should be to provide a better understanding of the fundamental forces and mechanisms governing protein crystal growth on Earth and in microgravity. While it may continue to be important to survey the crystal growth performance of a wide variety of proteins and techniques in space, it is becoming increasingly necessary as well to observe, measure, and manipulate crystal growth processes so that we understand what they entail. A systematic evaluation of which macromolecules derive the greatest benefits, and from which techniques, requires repetitive experiments with a single method to optimize growth parameters for specific proteins, as well as an expansion of experiments to a wider variety of proteins and methods. Analyses of chemical and physical characteristics of macromolecules that impact their organization into crystals merit support. Although a diverse mix of macromolecules might be desirable, even essential, in future crystal growth experiments, a systematic approach to these experiments is the only means of delineating the dominant factors involved in protein crystal growth in microgravity.

Automation and remote control of protein crystal growth will likely be needed for future microgravity experiments. Automated systems that permit dynamic monitoring and control of key variables such as temperature, protein concentration, ionic strength, $\mathrm{pH}$, and precipitant concentration will be useful for both groundbased and space experiments that are directed at better understanding of protein crystal growth processes. Nevertheless, at least for the near term, NASA may want to consider support for additional missions such as USML-1 in which an experienced observer is in control of iterative protein crystal growth experiments.

In summary, the success of initial experiments suggests that further research is needed and an expanded program of protein crystal growth experiments deserves support. It would be helpful if this research were to focus somewhat more sharply on gaining a clearer understanding of the physical and chemical phenomena involved in the nucleation and growth of protein crystals, in addition to developing an expanded range of techniques and methods to secure the benefits of microgravity growth for a broader range of macromolecular crystals. This implies increased basic research in protein crystal growth on Earth not only to expand understanding of the fundamental scientific principles, but also to support and develop the cadre of scientists needed to design and conduct the fundamental crystal growth experiments in microgravity.

\section{Separation and Purification of Biological Macromolecules and Assemblies}

Biological systems typically contain a large variety of proteins and nucleic acid molecules that have widely differing properties and that are often combined 
in macromolecular complexes, oligomers, and unique molecular assemblies such as viruses and ribosomes. Purification of macromolecules, complexes, or even cells is often a necessary first step to more detailed biological characterization and biotechnology applications. This is true whether isolation is directly from natural sources, such as blood sera, or from manufactured mixtures, such as culture media, fermentation products, hybridoma cultures, or synthetic peptide solutions. Furthermore, to exploit the potential of genetic engineering and engineered cells, it is necessary to purify products from the heterogeneous mixtures in which they are synthesized. Associated separation and purification challenges are often major obstacles to advanced biochemical studies and biotechnological applications.

Analytical separations commonly use electrophoresis in water-based gels to good advantage: gels of this type are generally recognized as providing the highest resolution for analytical separations of proteins and analyses of nucleic acid sequences. Density-driven thermal convection and sedimentation are well-known phenomena limiting the resolution of electrokinetic separations on Earth, and suppression of these phenomena in microgravity environments might be expected to eliminate the need for gels and make it possible to achieve the ultimate resolving power of electrophoretic experiments in free fluids. The results of electrophoresis experiments carried out in microgravity with well-characterized molecules and particles have shown that buoyancy-driven phenomena are diminished, but have revealed new phenomena at work that may affect purification processes. ${ }^{21}$ One of the significant findings has been the role of electrohydrodynamic effects in electrophoresis, where in certain experiments in microgravity they appear to significantly disrupt resolution. 22 More fundamental research, much of it in the fields of fluid dynamics and transport phenomena, is needed if investigators are to learn how to moderate or eliminate disadvantages due to electrohydrodynamic effects in microgravity. Further. while it is important to use microgravity to minimize negative effects caused by gravitational acceleration, it would also be useful to harness the unique features of microgravity fluid processes, such as virtually unlimited capillary rise or interfacial tension effects, for the enhancement of separation technology.

Separation processes also depend, in some cases, on subtle differences in the interactions between a molecule of interest and different solvents. Investigations of partitioning in ayueous polymer two-phase systems on recent shuttle missions have demonstrated the dependence of separation efficiency on several variables, including volume fractions of the component phases; container geometry; physical properties of the component phases, such as interfacial tension and relative phase viscosities; and surface tension interactions between aqueous phases and container walls. ${ }^{23}$ Some of these variables can be minimized; interactions with container walls, for example, may be reduced by special coatings.

The importance of improvements in resolution in chromatographic and electrophoretic separations to progress in the biochemical and biological sciences should 
not be underestimated. For example, biological sciences and biotechnology will be influenced significantly over the next decade by the mapping of the human genome-an effort that will require the isolation and purification of DNA fragments on a scale not encountered previously. For other applications, resolution requirements for protein purification have become increasingly stringent as the role of impurities in recombinant products has become more evident. A continued program of microgravity research in the separation and purification of biological macromolecules should be supported where it can contribute to improved techniques and improved results in terrestrial and microgravity enviromments.

\section{Cell Culture, Growth, and Differentiation}

The culture and manipulation of living cells, particularly the more fragile mammalian and plant cells, can be scientifically and technologically rewarding. but it can also be technically challenging. Some of these cell types, which naturally occur as part of specialized tissues and organs, are dependent for their survival and proper function on the environment provided by other cells and tissues. While the unique functions of differentiated cells make them especially valuable for research or for the generation of specific products. such cells, as a direct consequence of their interdependence, frequently exhibit sensitivity to shear, have complex metabolic requirements, and lose cell functions when cultured in artificial environments. Such sensitivities can complicate the utilization of these cells to generate desired products or responses. The culture of cells in microgravity offers a number of potential benefits: sedimentation and densitydriven convection are virtually absent, and changes in physical and chemical properties of the fluids constituting and nourishing the culture media can be exploited to benefit the culture system. The resulting environment may, therefore, be relatively quiescent and free of the steep velocity gradients in fluids and the sedimentation effects characteristic of ground-based cell culture systems. Improvement of the culture of shear-sensitive mammalian and plant cells would permit the manipulation of cells in ways that are difficult or impossible to accomplish on Earth. Such effects could be particularly important in processes that are dependent on cellular interactions, such as cell fusion or the assembly of three-dimensional multicellular systems.

Prototype culture systems have been developed that are compatible with microgravity operations and that simulate some of the fluid effects found in microgravity. Mammalian cell cultures have been used to test the feasibility and potential for extended microgravity research. Preliminary results indicate that culture devices developed with NASA support provide a low fluid shear environment with minimal sedimentation. ${ }^{24}$ Delicate mammalian cells have been cultured to very high densities in these instruments, and unique associations of cells into tissue-like aggregates have been observed. Limitations on the development of multicellular structures due to gravitationally induced disturbances 
have also been observed. Microgravity will enable maintenance of a culture environment that supplies minimum shear and maximum freedom for three-dimensional cellular association.

\section{Cell Fusion and Membrane Assembly}

The fusion of cells to produce viable hybrids-a low-frequency event with current terrestrial technologies-is another area that could benefit from the microgravity environment. Fused cells are used for genetic studies where natural crosses are impossible or impractical. Fusion is the key step to monoclonal antibody production. Cell fusion requires the interaction of two different cell types and the annealing of the cell membranes to yield one hybrid cell. The fusion of cell membranes is usually accomplished by treatment with electric current or chemicals, either or both of which may be harmful to the cell. The cells to be fused are usually of different densities, so sedimentation tends to separate the cells during the fusion process and leads to aborted fusion or cell death. Microgravity could reduce the tendency of the cells to separate due to sedimentation and density differences. This might permit a reduction in the intensity and duration of the high-frequency aligning field in the case of electrofusion and, possibly, in the concentrations of fusing agents used for chemically induced cell fusion. Similar considerations may be operative in the synthesis of liposomes and the deposition of biomembranes.

Further studies concerning the influence of a microgravity environment on cell fusion are needed. Results from sounding rocket and spacelab experiment $\$^{25,26}$ suggested that studies should be extended to typical mammalian fusion partners. The effects of such factors as cell viability, growth phase, composition of growth medium, and shear environment on the fusion process should be evaluated. Other technologies should be investigated in both ground-based studies and microgravity to determine whether improvements are observed in microgravity similar to those observed with electrofusion techniques. Basic research utilizing model artificial membranes and related biopolymer analogues may provide a better understanding of the processes and mechanisms at work in the fusion of natural cell membranes and could possibly lead to the development of more durable artificial membranes. A thorough characterization of the factors influencing fusion efficiency will improve Earth-based fusion technologies and will benefit the development of microgravity-based cell fusion activities.

\section{EXPERIMENTAL REQUIREMENTS}

Research areas in the biological sciences with expected sensitivity to the gravitational environment involve processes that typically take hours, days, or even weeks. The minimum duration of microgravity required for these experi- 
ments is, thus, greater than that available from drop towers, parabolic airplane flights, or suborbital rockets. If experiments in the biological sciences are to be conducted in microgravity, they must be carried out aboard the shuttle, the space station, or other orbiters such as free-flyers.

Little is known about the limits below which accelerations, or frequencies and durations of accelerations, must be maintained so that the biological effects of microgravity are not obscured. It must be true, of course, that if the overall acceleration environment of the experimental system is kept to a fraction of the stochastic acceleration environment of its molecular components, then interference with studies of biological effects of microgravity is avoided. In the case of the shuttle, it is known that the background acceleration environment in orbit. the so-called $g$-jitter, can reach levels that interfere with biological experiments. Acceleration environments will vary from vehicle to vehicle and acceleration limits will vary from experiment to experiment, so that the best match of experiment to vehicle will have to be judged on a case-by-case basis. It may also be that acceleration environments that are a constant fraction of unit gravity may be helpful to studies in some areas of the biological sciences. It is important, however, that acceleration environments be monitored, not only so that experiments can be interpreted properly but also so that realistic acceleration scenarios can be included in the experimental design.

\section{RECOMMENDATIONS AND CONCLUSIONS}

In support of the biological and biotechnical research areas described above, priority should be given in the order listed to the following research activities:

1. More research should be devoted to quantifying the role of gravity in protein crystal growth processes and to identifying those mechanisms affected by gravity.

2. Further experimentation is needed in both terrestrial and microgravity environments to develop new methods and materials that take advantage of mi-
crogravity for biochemical separations.

3. The potential advantages of the microgravity environment for the study of cellular interactions, cell fusion, and multicellular assembly processes should be explored to identify candidate cell systems that show maximum benefit from culture in a microgravity environment.

4. Cellular and biomolecular processes, structures, assemblies, and mechanisms that might be affected by gravity should be systematically assayed to explore the effects of microgravity on these systems. The objective should be a comprehensive database of cell types and their experiences in microgravity environments.

The first two topics have some demonstrated successes and the greater likelihood of future successes. The other two topics are exploratory. 


\section{REFERENCES}

1. Vanderhoff, J.W., and C.J. van Oss. 1979. Electrophoretic separation of hiological cells in microgravity. Pp. 257-273 in Electrokinetic Separation Methods, P.G. Righetti. C.J. van Oss. and J.W Vanderhoff, edh. Elsevier/North-Holland Biomedical Press, Amslerdam.

2. Cogoli, A. 1987. Cell cultures in space: From basic research to biolechnology II. Pp. 285290 in Proceedings of the Third European Symposium on Life Sciemes Research in Space, (iriz. Austrit, September 14-18. ESA SP-271.

3. Bugg. (.E. 1986. The future of protein crystal growth. J. Cryst (irowth. 76:535-544.

3. Buge. C. L.J. et al. 1989. Protein crystal growth in microgravity. Science, 246:651-654.

5. Mepherson, A A Greenwood, and J. Day. 1991. The effect of microgravity on protein rystal growth. Adv. Space Res., 1117):34,3-356.

6. Delucas, 1..l. et al. 1994. Recent results and new hardware developments for protein rystal growth in microgravity. J. Cryst. Growth, 135:183-195

7. DeLucats, L.J.. el al. 1993. Protein crystal growth results from the C'nited States Microgravity Laboratory-I mission. J. Phys. D: Appl. Phys., 26:B100-B103.

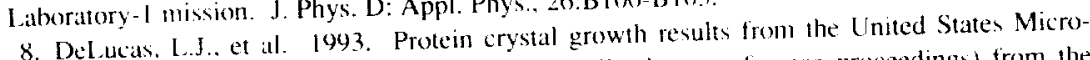
DaSA Reference Publication (conlerence proceedings) from the gravity Laboratory-1 Mission. NASA Reference Publicatille. Ala. September 22-24.

USML-1 and USMP-1 Joint L+1 Science Revlew. Huntsville, Ala., Septomer

9. Day. J.. and A. Mc.Pherson. 1992. Macromolecular crys

tional Microgravity Laboratory-1. Protein Science. 11:1254-1268.

10. McPherson. A. 1992. Effects of a microgravity environment on the Symposium on Materials

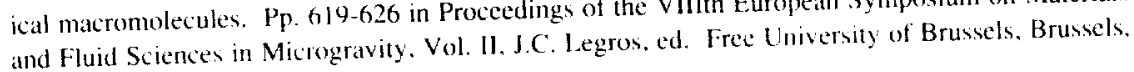
Belgium.

11. MePherson, A. 1993. Virus and protein crystal growth on Garth and in microgravily. J.

Phys. D: Appl. Phys., 26:104-112.

1992 Macromolecular crystial growth experiments on Interna12. Dily. J., and A. McPhory-1. Protein Science, 11:1254-1268.

1994. Protein crystal growth in microgravity-temperature-induced

13. Long. M.M.. et al. 1994. Protein eryal grow International Symposium of Microgravity large-scale crystallization of insulin. Proceedings of the Internations 202.

14. Stoddard B.L. R.K. Strong, G.K. Farber. A. Arrott and (i. Petsko. 1991. Design of apparaexperiments to determine the effect of microgravity on the crystatlization of biological

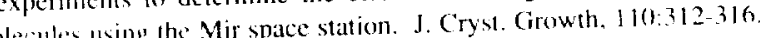

15. Sirong. R.K. B.L. Stoddard, A. Arrott, and G.K. Farber [992. I ong duration growth of

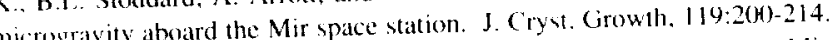

protein crystal in microgravity aborard he Mir space stath Jesults from the United States Micrograv-

16. Delucas. L.J., el al. 1993. Protein crystal growth resulto Irom.

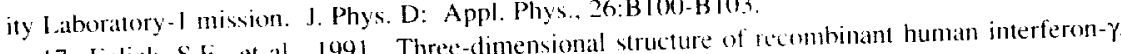

17. Fallick, S.E... et

Science, 252:698-702.

18. He, X.M and D.C. Carter. 1992. Atomic structure and chemistry of human serum albumin.

Nature. $358: 2(14-215$

19. DeLucas, L.J., et al. 1993. Protein crystal growth results from the United States Microgravity Laboratory-I mission. NASA Reference Publication (conference proceedings) from the USML-1 ity Laboratory-1 mission. NASA Reteren. Huntsville. Ala., September 22-24.

20. Day, J, and A Me Pheron 1992. Macromolecular crystal growh experiments on Interna-

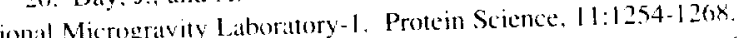

W 1987 . Continuous flow electrophoretic separation of proteins and cells 21. Hymer, W.C., et al. Aell Biophysics, 10:61-85. 
22. Rhodes, P.H. R.S. Snyder, and (i.O. Roberts. 1989. Electrodynamie distortion of sample streams in continuous flow elecorophoresis. J. Colloid Interface Sci., 129:78-90).

23. Van Alstine, J.M., S. Bamberger. J.M. Harris, R.S. Snyder, J.F. Boyce, and D.E. Broohs. 1990. Phase partitioning experiments on Shutle flight STS-26. Pp. 399-407 in Procededings of alic VIth Furopean Symposium on Materials and Fluid Sciences in Microgravity, Oxford, Lnited Kingdom. September 10-15. ESA SP-295.

24. Cimuinder. F.K... and A. Cogoli. 198x. Cultivation of single cells in space. Applied Microgravity Technology 1:115-122.

25. Baumann. T., W. Kreis, W. Mehrle, R. Hampp, and E. Reinhard. 1990. Regencration and characterization of protoplast-derived cell lines from Digitalis hanata EHRH and Digitalis parmera L. suspension cultures after electrofusion under microgravity conditions. Pp. 405-410 in Procedings of the Fourth European Symposium on Life Sciences Rescarch in Space. Trieste, Ititly. May 28 June 1. ESA SP-307.

26. German Ministry of Research and Technology (BMFT). 1987. Procecdings of the Norderney Symposium on Scientific Results of the German Spacelab Mission DI, P.R. Sahm. R. Jansen, and M.H. Keller, eds. Wissenschaftliche Projektfuerung DI. Cologne, Germany. 


\section{6 \\ Materials Science and Processing}

This chapter discusses the areas of microgravity research generally identified as materials science. As organized in this report, they include metals and alloys, organic materials and polymers, the growth of inorganic single crystals, the growth of epitaxial layers on single-crystal substrates, and ceramics and glasses.

\section{METALS AND ALLOYS}

\section{BACKGROUND AND INTRODUCTION}

One of the major desires of modern materials scientists and physical metallurgists is to understand the formation, structure, and properties of materials from the atomic and molecular levels $(0.1$ to $1 \mathrm{~nm})$ up through the mesoscopic level $(0.1$ to $100 \mu \mathrm{m})$. Many physical properties of materials are influenced directly by this microstructure (i.e., the morphology, size scale, spatial distribution, interface characteristics, and composition of different phases or structures that are present within a material). The relationship between the microstructure of a material and its physical properties and the manipulation of various processing parameters to obtain a given microstructure constitute key elements in the modern study of materials.

Metallurgy has evolved over the millennia from a purely empirical art to a modern branch of materials science, with considerable progress being made in understanding some of the simpler metallurgical systems and processes from first 
principles. A key to this progress has been the improved ability to control the solidification process, an important scientific feat that has come about through an improved understanding of the interrelationships between heat and mass transport phenomena occurring in the melt and the kinetics of the solidification interface.

Most metals and alloys are solidified from a fluid state. Since it is often desirable to mix two or more components to obtain alloys or composites with improved physical properties, the solidification process becomes complicated by the fact that different atoms do not generally enter the solid in the same proportion as they occur in the melt. This chemical segregation produces local variations in composition in the melt that, in turn, change the temperature at which various fractions of the solid will form. The situation described here is complicated further by the presence of convective and advective liquid flows that also modify the local composition and temperature of the melt. The microgravity environment, by reducing these gravity-driven phenomena, clearly offers new opportunities to metallurgists to develop and enhance control of materials processing.

The microgravity environment provided by an orbiting spacecraft offers new opportunities in control of the solidification process. Reduction of convective velocities permits, in some cases, more precise control of the temperature and composition of the melt. Body force effects such as sedimentation, hydrostatic pressure, and deformation are similarly reduced. Weak noncontacting forces derived from acoustic, electromagnetic, or electrostatic fields can be used to position specimens while they are being processed, thus avoiding contamination of reactive melts with their containers.

To accomplish the objectives discussed above, it is necessary to conduct a series of carefully chosen, well-conceived experiments that clearly delineate the advantages and limitations of microgravity research. These experiments should be designed to produce new results that could not have been obtained by terrestrial experiments.

\section{FUNDAMENTAL RESEARCH AREAS-METALS AND ALLOYS}

\section{Nucleation and Metastable States}

In order for a material to transform to a more ordered phase (vapor to solid or liquid to solid), it is first necessary to form an aggregate or cluster of molecules above a critical size to initiate the process. Such an aggregate may (by heterogeneous nucleation) form on a foreign surface, such as a container wall or a freely floating mote, or it may form spontaneously (by homogeneous nucleation) from random internal thermodynamic fluctuations. Homogeneous nucleation can occur only if the melt is cooled far below its normal freezing temperature without solidifying. Heterogeneous nucleation almost always occurs first, provided there are impurities that can act as nucleation sites. Understanding and 
controlling phase nucleation are extremely important for control of the overall solidification process.

For example, if a fine-grained casting is desired, one would try to produce a very large number of crystal nuclei and, ideally, distribute them randomly throughout the melt. Gravity-driven convection plays an important role in this process, as was demonstrated in a series of early experiments at reduced gravity using Space Processing Applications Rockets (SPARs) and at increased gravity using a centrifuge. This research is an example of how microgravity experiments may be used to elucidate the essential features of a solidification process and to suggest better control strategies for use on Earth-in this case, by enhancing convection or mechanically stirring the melt to distribute the crystal nuclei.

It is often desirable to suppress, or even avoid nucleation entirely, in order to be able to supercool a melt to a temperature well below its normal thermodynamic freezing point. Solidification of deeply undercooled metallic melts is usually initiated by a single nucleation site, and freezing tends to be rapid. These melts generally produce a refined dendritic microstructure, often exhibiting enhanced mechanical properties. If the solidification is rapid enough, the solute atoms will not have sufficient time to arrive at positions of lowest energy (i.e., near their equilibrium configurations); metastable crystalline or even amorphous phases can thus be produced. A metastable phase can have a crystalline structure different from that of the equilibrium phase, which can greatly alter some of the material's physical properties in technologically significant ways. Perhaps the best-known metallurgical example of a metastable crystalline phase is steel, which is hardened by formation of the metastable phase martensite. Martensite is a nonequilibrium tetragonal phase containing carbon and iron that forms upon rapidly quenching austenite, which is a cubic, equilibrium phase consisting of carbon dissolved in the face-centered cubic form of iron.

An amorphous phase may be thought of as a liquid-like structure, frozen in place, that lacks long-range crystalline order. Ordinary soda-lime silica glass is a classic example of an amorphous engineering solid. Amorphous materials tend to be highly resistant to chemical attack because there are no crystalline grain boundaries on which most chemical reactions occur. Similarly, glassy amorphous structures can be achieved in some metallic alloys by rapid solidification. One example is Metglas ${ }^{\circledR}$ _a product developed by Allied Signal Corporation that consists mainly of iron. silicon, and boron. The absence of grain structure makes it extremely easy to magnetize and demagnetize Metglas ${ }^{(B)}$ with little magnetic hysteresis loss, thus making this material useful in transformer cores, where it provides greater efficiency than the conventional, crystalline ironsilicon material. By contrast, a new rapidly solidified iron-boron-neodymium alloy, developed recently by General Motors Corporation, is an extremely good 
permanent magnet, with high coercivity, because its fine grain structure tends to "pin" the magnetic domains and prevent demagnetization. These examples demonstrate how crucially the physical properties and performance of a material depend on its microstructure.

The ability to melt, supercool, and subsequently solidify alloys without a supporting container or crucible removes a major source of impurities and heterogeneous nucleation sites, thus permitting very large undercoolings to be achieved. On Earth this can be accomplished for small metaltic samples by levitating them on a gas-film or in an electromagnetic field and then releasing the molten droplets into a long, vertical, evacuated tube and allowing them to solidify during free-fall.

These techniques have been used extensively over many years but have severe limitations. It is not possible, for example, to control the heating and levitating force independently in an electromagnetic levitator. Use of a quench gas to cool the sample often promotes surface nucleation. There is also some evidence that flows in the melt driven by the induced alternating currents may stimulate nucleation and limit the amount of undercooling. These difficulties can be overcome in a drop tube, but it is not yet possible to observe the falling droplet closely enough as it cools in order to make accurate temperature measurements. Also, the sample volume and the amount of undercooling are limited by the time available for the fall (typically a few seconds). In reduced gravity, the sample may be positioned by a much lower induced current or may even be allowed to float freely. This significantly decouples the heating field from the levitation field and greatly reduces the amount of stirring in the melt. The temperature may then be measured optically during cooling and the solidification process, and thermophysical properties such as specific heat, heat of fusion, and thermal diffusivity can be determined calorimetrically.

Initial microgravity experiments on metals and alloys should focus on identifying factors that limit the degree of undercooling, such as the amount of superheat required to dissolve or destroy potential nucleation sites in a melt, the use of so-called fluxing agents to remove impurities and prevent oxidation, and the effects of stirring and melt shear on promoting heterogeneous nucleation. Once techniques for obtaining large undercoolings have been determined for an alloy, the emphasis of subsequent experiments might be on forming metastable and amorphous phases from deeply undercooled melts and determining their properties. It may even be possible to perform critical supercooling experiments to evaluate various theories of nucleation that have never been verified experimentally. Convenient methods for obtaining extremely low partial pressures of reactive gases, such as oxygen, must also be developed since oxides and other reaction products will form rapidly on molten samples and, in some systems, act as effective nucleation sites. 


\section{Prediction and Control of Microstructure}

Microstructure can be manipulated to a significant degree by controlling the rate at which solidification takes place and the temperature distribution in the region of the crystal-melt interface. This is often accomplished by the process of directional solidification, in which heat is extracted unidirectionally either by placing the bottom of the crucible containing the molten specimen on a chillblock (gradient freese method) or by slowly lowering the crucible through a temperature gradient (Bridgman method). ${ }^{*}$ If the temperature everywhere in the melt is above the local freezing (liquidus) temperature, a smooth, almost featureless, solidification front will result. This is desirable for growing single crystals or for producing aligned composites. However, in virtually all alloys, the atoms are incorporated into the solid phase in proportions different from the melt. This leads to a region ahead of the freezing interface in which the solute concentration is altered from its mean composition. Within this thin region or boundary layer, the equilibrium freezing point in the melt can be lower than the freezing point of the original alloy. If the temperature gradient in the melt is not steep enough, which is generally the case in a slowly frozen casting, a situation known as "constitutional supercooling" will arise, in which the melt ahead of the interface is below its equilibrium freezing temperature. At the onset of constitutional supercooling, the initially smooth interface becomes unstable and forms a series of protuberances or cells. At higher degrees of constitutional supercooling, the interface breaks down completely as these cells and bumps elongate and develop side branches to form tree-like structures called dendrites. When this happens, partial solidification proceeds over a substantial distance ahead of the totally frozen material. The region between the advancing solid and dendrite tip is called the "mushy" zone because it is composed of a fine, micrometer-length scale mixture of liquid and solid. The heat and mass transfer processes that take place in this two-phase "mushy" zone determine the microstructure of the solid.

Although these processes are understood qualitatively, the detailed quantitative descriptions required to control the microstructure remain poorly understood. A great deal of theoretical work has been devoted to obtaining accurate descriptions of dendrite growth rate, shape, and primary and secondary arm spacing-all as functions of the solidification and materials parameters. ${ }^{1}$ Generally such fundamental theories ignore the important effects of convection in order to remain tractable. Convective flows are usually unavoidable in Earth gravity because of the lateral density gradients that must exist as a result of the solute rejection described above. In fact, short-duration, low-gravity experiments carried out on sounding rockets and in aircraft flying parabolic (low-gravity) trajectories have shown evidence that both the primary and secondary arm spacings change as the force of gravity is altered. ${ }^{2}$ Therefore, it was considered scientifi-

\footnotetext{
*For a discussion of both methods, see section below, Growth of Inorganic Single Crystals.
} 
cally important to test the first-order fundamental theories that ignore convection in a microgravity environment before adding modifications to correct for convective effects that are encountered in terrestrial processes. Such a critical test, known as the Isothermal Dendritic Growth Experiment (IDGE), was carried out recently aboard the United States Microgravity (USMP-2) mission launched March 3, 1994. Preliminary results obtained from the IDGE show that the terrestrial dendritic growth data used previously to test diffusion-based theories were, in fact, heavily corrupted by buoyancy-induced convection. As a result, these fundamental theories, each one purportedly providing the basis of dynamical pattern selection, now remain in question. ${ }^{3}$

The discussion, thus far, applies only to alloy systems that have one solid phase, (i.e., to solid solutions in which the atoms in the solid are completely miscible or to dilute alloys in which the minor constituent does not exceed the limit of solid solubility). Most alloy systems of practical interest, however, display only partial miscibility in the solid state, a situation that produces a more complicated behavior involving polyphase reactions. An important class of materials exhibiting this behavior is the eutectics in which a melt consisting of a homogeneous solution of the two components solidifies to form a solid with segregated regions of quite different compositions. A good example of this type of behavior occurs in cast iron, whose melt consists of a solution of iron and carbon. On freezing, flakes or nodules of almost pure graphite form in a crystalline matrix of nearly pure iron. The morphology or form of these graphite particles largely determines the mechanical properties of the material. Despite many years of intensive research, all the kinetic and thermodynamic factors that determine the graphite morphology are still not understood.

By carefully controlling the direction in which heat is extracted (directional solidification), interesting, controlled, two-phase microstructures can be produced in a variety of eutectic systems. In some systems, it is possible to grow thin rods of one composition and crystal structure that are embedded in a matrix of yet another composition and crystal structure, with both aligned along the direction of heat flow. In other systems the resulting structure comprises a series of alternating thin slabs, or lamellae, of the two compositions. These structures are termed "in situ composites" and often exhibit highly anisotropic properties because of the aligned microstructure. For example, some turbine blades manufactured by directional solidification for high-performance jet engines exhibit this aligned structure and show improved strength and creep resistance. In another example, the theoretical magnetic coercivity in a manganese-bismuth eutectic alloy was approached by using directional solidification to match the eutectic rod diameter to the size of a single elongated magnetic domain.

The classical theory of eutectic spacing selection assumes only diffusive species transport, because convective effects were thought to be unimportant on the length scales involved. However, in a series of microgravity experiments for the manganese-bismuth eutectic, it was found that the rod diameter and spacing 
were considerably smaller than predicted from this theory. Interestingly enough, ground control experiments, which had convective flows, yielded results that agreed well with the classical theory. ${ }^{4}$ Growth in strong magnetic fields, on the other hand, which suppress convective flows, produced results similar to those from the spaceflight experiments, clearly indicating that convective effects are in fact important in the process. European experimenters on Spacelab-1 and D-1 have found similar results with other eutectic systems, agreement with the classical theory in other systems, and even larger spacings than predicted by classical theory in yet other systems. Obviously, there is still much to be learned about the science of eutectic solidification.

Another effect that is important in the evolution of the microstructure of an alloy is the phenomenon of ripening or coarsening. It can be shown from thermodynamics that the melting point of a solid particle is reduced slightly by the curvature of its surface (Gibbs-Thomson effect). This effect comes about because of the interfacial energy change associated with the motion of the curved surface during melting or freezing. A planar surface (zero curvature) would not experience a change in energy on melting or freezing. Given a distribution of solid particles with varying curvatures in a melt, the larger particles will grow at the expense of smaller particles as the system tends to lower its free energy by reducing its total surface area. This effect, known as Ostwald ripening or phase coarsening, is important in a large class of dispersion-hardened alloys in which fine particles are either added to or caused to precipitate from the melt during solidification. Since the strengthening effect of the dispersed particles diminishes as the particle size increases, the process of coarsening must be understood and controlled. Similarly, in castings and weldments, dendrite arms coarsen by the same process, which again affects the final microstructure and properties.

The classical theory of Ostwald ripening was developed in 1961. An approximate solution was obtained that is valid in the limit of zero volume fraction of solid particles. In metallurgical applications, however, the dispersed phase makes up a significant fraction of the system. Also, it appears that neighboring particles interact strongly with one another, an effect that is not accounted for in the approximation used in the classical theory. A number of attempts have been made recently to modify the classical theory, but so far none of these theories can predict experimentally observed phenomena. This might be due to the fact that none of the theories considers convective transport of the continuous liquid phase (because the fluid channel sizes are small); yet it might be an important factor, as well as an additional complication.

Well-defined microgravity experiments are needed to confirm the quantitative aspects of the underlying physics in the theory of Ostwald ripening under the conditions of pure diffusive transport. Such experiments need to be performed before the effects of convection and fluid motions can be incorporated into the theory. 


\section{Phase Separating Systems and Interfacial Phenomena}

Another class of polyphase materials that is of interest to microgravity research involves monotectic systems, which are characterized by a compositional region of liquid-phase immiscibility. At any given temperature (below the critical temperature) a range of compositions is always encountered for which the melt will separate into two immiscible liquids. Since the two liquids are of different composition, they will invariably have different mass densities. In Earth gravity the two liquid phases will stratify before they can be frozen, resulting in a highly chemically segregated solid. It was reasoned that this buoyancy-driven sedimentation could be avoided by solidifying such systems in microgravity. When actually performed, such experiments have been only partially successful in producing the expected fine dispersions uniformly distributed throughout the final solid. ${ }^{5}$ These experiments indicate that effects other than gravity-induced buoyancy become important in the solidification of monotectic systems. An extensive series of ground-based experiments, using systems that have similarly configured (monotectic) phase diagrams, and which in some instances can be made neutrally buoyant, has uncovered a rich variety of interfacial energy-driven effects (such as critical wetting). These monotectic alloys exhibit a variety of interesting microstructures, which have been scientifically classified through the help of microgravity research."

A clear example of the dominating influence of interfacial energy effects occurs in monotectic alloy systems below the critical temperature. Over a temperature range below the critical temperature, one of the liquids always exhibits perfect (or critical) wetting behavior, so that one fluid phase essentially encapsulates the other and, even more curiously, the wetting phase will perfectly coat any container or surface that it contacts. If final freezing occurs within the temperature range of critical wetting, then massive phase separation will be produced in the microstructure. Alternatively, if the temperature for the monotectic reaction, which produces a solid- and liquid-phase pair from a different liquid composition, falls below the critical range, then useful (dispersed) microstructure control is possible. A continuous, well-aligned two-phase structure with uniform spacing between phases can often be achieved in a directional growth process. ${ }^{7.8}$ In the latter case, melt flows driven by the temperature and composition dependence of surface energy can become dominant. The minority liquid phase can form droplets that will migrate in a thermal gradient (thermophoresis) as well as in a composition gradient. Larger droplets move faster than smaller ones, so that the smaller ones will be overtaken and agglomerated with the bigger ones. Again, massive phase separation will result. In fact, depending on the alloy system. thermal gradients as small as $1 \mathrm{~K} / \mathrm{cm}$ in low gravity can have the same motive effect as Earth gravity in causing massive segregation.

A second case of dominating interfacial factors occurs in containerless processing, where the extent of wetting between the solid(s) forming from a melt 
controls the degree of undercooling and the resulting microstructures. Brazing. soldering, and welding operations represent additional technologically important processes that are influenced greatly by interfacial phenomena. Here both wetting and surface tension-driven flows can be primary influences in achieving or not achieving success.

It is clear that interfacial phenomena are common to numerous metals processing strategies. Although limited studies of interfacial effects are always possible on Earth using density-matched immiscible systems, density matching per se under the influence of terrestrial gravity can be accomplished only at a single temperature. Thus, the microgravity environment provides a unique opportunity to study and quantify a range of interfacial phenomena in order to suggest better materials processing strategies on Earth and under microgravity conditions.

\section{Heat and Mass Transport}

The freezing temperature of any alloy is dependent on composition, and a freezing solid tends to accept one component of a melt more readily than the other components; thus, the first-to-freeze solid will always have a composition different from that of the remaining solid. The rejected component sets up a compositional gradient in the melt at the solidification interface. With gravity present, any lateral density gradient resulting from composition or thermal gradients will produce prompt convective stirring. Convective mixing of the enriched solute rejected from a growing crystal-melt interface with the bulk melt causes a spatially varying composition (i.e., segregation) to occur throughout the solidified specimen. This phenomenon is called macrosegregation. When the mixing rate varies in time, the temporal variation results in a solid with a profile that might exhibit bands of composition that vary along its length.

Macrosegregation can be controlled to some extent by extracting heat unidirectionally from the bottom of the specimen to minimize lateral thermal and solutal gradients. However, if the rejected component of the melt at the interface is less dense than the bulk melt, a phenomenon known as a double-diffusive convection can develop. Channels or plumes of the lighter melt will rise from the interface, resulting in solidified regions with a grossly different composition (known as "freckles").

Macrosegregation also plays a significant role in the formation of in situ composites with aligned microstructure. Even with a well-controlled directional solidification process such as the Bridgman method, lateral thermal gradients occur that will produce some convective stirring. Thermosolutal convection occurs in Earth gravity if the rejected component is less dense than the bulk melt. Strong magnetic fields can slow these convective flows but cannot eliminate them. Thus, magnetic fields are generally of limited effectiveness for controlling macrosegregation. Microgravity offers the opportunity to produce unique microstructures in a variety of systems (e.g., off-eutectic compositions, mono-

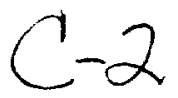


tectics, and peritectics) that tend to phase-separate in normal gravity, as well as in solid solutions that are subject to double-diffusive convection. Liquid-phase sintering of dense tungsten particles with lighter molten transition alloys (typically $\mathrm{Fe}-\mathrm{Ni}$ ) is a good example of an important terrestrial process that would be assisted if performed in a microgravity environment.

Experiments in microgravity are being flown by NASA to test theories of solidification, seeking a more basic understanding of the way in which microstructures are formed. Since the solidification process involves the transport of heat and mass and a moving phase boundary, one way to test the theories is to encode them as a mathematical model and then compare the predicted thermal and solute fields with those observed experimentally. This allows evaluation of competing theories using the same experimental data.

Most of the mathematical models that have been developed thus far consider only steady accelerations. Additional modeling development efforts are clearly needed to account for transient, random, and oscillatory accelerations (collectively termed " $g$-jitter") and their effects on heat and mass transport. The ability to design and interpret scientific experiments in the microgravity program is often predicated on the available knowledge of the relevant solute and temperature fields. Mathematical and numerical models are an indispensable tool to augment experimental measurements for this purpose. There are two distinct ways to use the mathematical models that describe the physical transport processes of interest: (1) to design and then theoretically interpret scientific experiments performed in microgravity, and (2) to obtain values for certain thermophysical properties, which are difficult to measure terrestrially, by using microgravity experiments, and then employ these data to improve model-based predictions of terrestrial processes used in industry. In order to improve the chances of a successful flight experiment and take maximum advantage of the relatively few flight opportunities that will be available over the next decade, candidate experiments must be carefully modeled mathematically for both terrestrial and microgravity conditions. Even for a six-order-of-magnitude reduction in gravity, significant flows can still result if the residual gravity vector is perpendicular to a large density gradient. This can easily occur in a directional solidification experiment unless care is taken to ensure that the residual acceleration environment is controlled and that the experiment is configured to minimize its effects, for example, by application of a strong magnetic field.

\section{Thermophysical Properties}

Another serious deficiency in our ability to model solidification processes is a paucity of accurate thermophysical property data for many alloys in the molten state. This is a problem not only for scientists modeling microgravity experiments, but also for many industrial researchers who are using the computational methods made possible by supercomputers to model the solidification of com- 
plex castings. The lack of high-temperature thermophysical data is due in part to the extreme difficulty of making accurate measurements on melts under terrestrial gravity. Recent European experiments on Spacelab-1 and D-1 have shown that diffusion coefficients for a variety of molten alloys measured in space differ considerably from the accepted values obtained on Earth. This situation is believed to occur either because of wall effects (such measurements are often made in capillary tubes) or because of uncompensated convective transport, which is virtually impossible to avoid in a terrestrial setting. Also, the effect of thermodiffusion (Soret-Dufour effect) was found in space experiments to be as much as an order of magnitude larger than previously estimated. No accurate measurements had been made of this effect for solidification processes conducted on Earth. This effect, which causes a mixture of atoms to become separated in a thermal gradient according to their atomic mass, now appears to play a more important role in mass transport in many terrestrial processes than had been realized heretofore.

The following are some thermophysical properties, ${ }^{9}$ only a few of which can be advantageously measured in microgravity, that are of interest in the development of reliable models for metals processing:

- Emissivity, electrical conductivity, and optical properties;

- Calorimetry including specific heats and heats of mixing, formation, and transformations;

- Transport coefficients including thermal conductivity, viscosity, and diffusion constants:

- Density data;

- Thermodynamic moduli, including thermal expansion coefficients and compressibility;

- Vapor pressures and activity coefficients;

- Surface tension and interfacial energies; and

- Equations of state.

\section{FUTURE SCIENTIFIC DIRECTIONS-METALS AND ALLOYS}

As indicated in the previous section, a number of important scientific problems in metallurgy are being addressed by experiments currently sponsored by NASA's microgravity science and applications program. However, other important areas can be identilied that are just beginning to be developed. These areas include powder metal processing, electrolytic processes, joining, ultrapurification, ultrahigh-vacuum processing, nanomaterials, and cuprate superconductors.

Powder metallurgy represents an important technology for processing many dense refractory metals or materials that tend to phase-separate when melted in unit gravity. Indeed, some of the gravity-related problems discussed previously can be avoided by standard powder processing: for example, by compacting a 
mixture of the components in the form of a fine powder and heating it just below the melting point for some time (sintering). Theoretical density can often bc achieved by applying high pressure (hot isostatic pressing) or by raising the temperature so that one of the components melts (liquid-phase sintering).

Since the powder metal process is designed ab initio to minimize the effects of gravity, one would not expect to see any large differences between Earth- and space-processed samples. Rapid particle growth during liquid-phase sintering is an anomalous processing effect that might arise from sedimentation-induced convection or from some unidentified interfacial phenomenon that promotes coalescence. Well-designed microgravity experiments should provide some valuable insight as to the sensitivity of such powder processes to these effects.

Little has been reported in the United States concerning electrodeposition, electropolishing, or corrosion in reduced gravity. Convection obviously plays an important role in such processes because of the compositional changes in the electrolyte in the vicinity of the electrodes. A recent German experiment reported the formation of amorphous nickel in a high-current-density, short-duration experiment on a sounding rocket, whereas the ground control experiment yielded crystalline nickel under the same conditions (except for the presence of gravity). It would be useful to compare the morphologies of various electrodeposits under different convective conditions. In the virtual absence of convection, it might be possible to produce highly ramified, fragile, dendritic structures with extremely large surface areas that could be useful as catalyst substrates. An understanding of how reduced convection causes this to happen may permit development of new terrestrial processes.

Except for a few early experiments on Skylab, low-gravity experiments in welding, brazing, or soldering have not been undertaken by U.S. investigators. The early metal joining experiments showed not only that welding and brazing were certainly feasible in microgravity but also that significantly different microstructures were formed in the low-gravity welds. The altered microstructure has never been satisfactorily explained. The joining of metals in space also represents a technology that should be developed to support the construction and repair of the large orbital structures necessary for continued human presence in space.

\section{RECOMMENDATIONS AND CONCLUSIONS}

Carefully designed and scientifically well-conceived experiments on metals and alloys are needed to produce high-quality data and materials derived from microgravity research. Such experiments are not useful unless they produce results that cannot be obtained in terrestrial studies. Topics in the metals and alloys area that might benefit from a focused effort in microgravity research follow in rank-order: 
1. Nucleation kinetics and the achievement of metastable phase states, such as metallic glasses and nanostructures, are areas of scientific interest that would benefit from achieving deep supercooling in the microgravity environment by elimination of container surfaces and from reduction of melt flows due to buoyancy-driven convection.

2. Microgravity experiments on Ostwald ripening and phase coarsening kinetics would add quantitative, fundamental information on the key metallurgical issues of interfacial dynamics during thermal and solutal transport and on the question of microstructure evolution in general.

3. Observations of aligned microstructures processed reproducibly under quiescent microgravity conditions should help to provide well-defined thermal processing limits to polyphase directional solidification of eutectics and monotectics, which comprise wide classes of technologically important alloys and composites.

4. Studies of the formation of solidification cells and dendrites under welldefined microgravity conditions can add to our expanding knowledge of complex metallurgical pattern formation and, more generally, of the fundamental physics of nonlinear dynamics. Such studies, to be successful, require the most demanding control of conditions such as temperature. thermal gradients, growth speed, and alloy composition. Microgravity conditions can be useful in these instances for the pursuit of sophisticated tests of theory and the quantification of metallurgical pattern dynamics.

5. Some thermophysical properties can be measured advantageously in microgravity. Accurate data on these properties, frequently essential for the modeling of metallurgical processes and materials responses, are often not available from standard terrestrial measurements.

\section{POLYMERS}

\section{BACKGROUND}

Polymeric materials processing remains a relatively understudied discipline within the Microgravity Science and Applications Division (MSAD) of NASA. Part of the reason for this is that most engineering polymers are too viscous for either buoyancy-driven convection or gravity-driven particle motions to be important or play a role within the limited time scale allowed by most ordinary processing events. However, a wide range of lower-molecular-weight polymers exist that are responsive to the gravitational environment.

Latex suspensions, for example, can exhibit shear viscosity values about the same as those for water and, consequently, may be used as polymerizing organic systems for the study of gravitationally mediated transport phenomena, including molecular diffusional processes. Thus, more careful consideration reveals a 
rationale for studying organic systems in the low-gravity environment that is not dissimilar to the reasons supporting scientific uses of microgravity research for other materials (e.g.. inorganic crystal and protein crystal growth). For these reasons, the microgravity environment may, ultimately, also be useful in addressing certain fundamental issues of interest to the organic and polymer areas of the materials science community. Polymeric materials have a number of special properties that may be of interest as subjects of fundamental microgravity study. For example, polymers and many organic molecular melts tend to have strong molecular orientational and conformational constraints and, consequently, supercool strongly before crystallizing from a solution or melt. Thus, the resulting morphology and texture of some polymeric solids are responsive to gravitationally driven shear flows in the melt during solidification and to the heat and mass transfer processes occurring as a result of these flows. Additionally, polymerizations may be affected by gravity in at least two other significant ways: (1) the termination of radical polymerization requires the contact of two radical-containing polymer chain ends; gravity-driven convection provides an efficient method of terminating them. A consequence is that one could expect that polymer molecules grown to termination in microgravity might have longer average chain lengths and higher molecular weights. (2) During copolymerizations, convection flows reduce the thickness of the boundary layer of preferentially rejected monomers from near the chain ends, so copolymers reacted in reduced gravity tend to produce materials that have greater uniformity in their distributions of average molecular weights and chain lengths. ${ }^{10}$

Finally, in recent years there has been growing interest in the field of nonlinear optical (NLO) materials. Important applications such as optical switching. optical communications, and optical computing all require devices containing NLO materials that possess large nonlinear optical responses. At present, however, NASA has had only limited involvement in developing and supporting research in this field. This is most likely due to the paucity of prior research emphasizing space-based studies on NLO materials. Indeed, there now appear to be two major areas recognized in which NLO research advances could be of direct importance to NASA. One is in optical communications, where novel NLO devices can be used in laser communications satellites deployed in space. The second is in microgravity research, where microgravity might lead to improved NLO materials and processing and, ultimately, to devices with superior optical properties. This would be especially useful if it led to improved processing on Earth.

\section{EARLY STUDIES AND THEIR CURRENT RELEVANCE}

Closely controlled polymerizations carried out in a solid polymer/liquid monomer matrix often are affected adversely by particle collisions that occur in response to gravitationally driven sedimentation and stirring. For example, stirring. applied 
during ground-based polymerizations, is often needed to maintain the dispersion stability of suspensions that sediment under the action of gravity. Agitation from stirring, however, causes particle-particle collisions that result in uncontrolled flocculation and agglomeration. It was recognized quite early in the development of microgravity flight programs that reduction in the gravity force could be used to widen the parameter range of emulsion stability for latexes and other polymerizing systems; such studies were remarkably successful. Specifically, it has been shown, through both ground-based and orbital flight experiments, that large populations of nearly monodisperse, latex microspheres (in the 20 - to 30 - $\mu \mathrm{m}$ size range) could be formed by low-gravity processing of various polymer solutions. " The size distributions of the microspheres grown in microgravity were sufficiently monodisperse and well characterized to justify their initial use as practical laboratory length scale calibration standards for electron microscopy and $x$-ray diffraction analysis. These early space-grown latex microspheres also proved useful as test markers for measuring the efficacy of separation techniques such as electrophoresis and electroosmosis. ${ }^{12}$ Although originally conceived as a fundamental investigation of the kinetics of microsphere formation in microgravity, these early polymer experiments were redirected by MSAD to be more applied in their nature. One area of potential application for highly monodisperse microsphere technology may be in the production of monodisperse spherical dye lasers in the $100-\mu \mathrm{m}$ size range. To improve further upon the degree of monodispersity, sphericity, and size range achieved in the early low-gravity microsphere studies, however, significantly increased understanding of the physics of emulsion polymerization may be required.

Polymer thin films prepared with fewer defects and more uniform thickness also provide superior optical devices. Such improved films have been prepared by electrochemical polymerization in microgravity. Elimination of solutal convection under electrochemical deposition conditions in low gravity was observed ${ }^{13.14}$ using a laser shadowgraph/schlieren technique to observe the concentration gradients in electrodeposition experiments on a $\mathrm{KC}-135$ aircraft in parabolic tlight. Shadowgraphs showed the absence of solutal "plumes" at the electrode surface in low gravity. The feasibility of using electrochemical techniques to assist and stimulate polymerization to prepare NLO polymer thin films for use in devices was demonstrated ${ }^{15}$ with prepared films of polythiophene and a homologous series of thiophene-based polymers that had NLO conversion efficiencies among the largest ever observed for polymers and comparable to the optical performance obtained for the polydiacetylenes. ${ }^{16}$

Recent research ${ }^{17-20}$ indicates that better-quality thin films for use in NLO devices might be obtained by closed-cell physical vapor transport (PVT) in microgravity. In the PVT process, the source material is sublimed in an inert gas or dynamic vacuum and allowed to convect or diffuse down a thermal gradient and condense at a crystal or thin film growth interface. The advantage of thin film growth in microgravity is that it provides the opportunity to eliminate buoyancy-driven convection and approximate diffusion-limited growth. 
Physical Vapor Transport of Organic Systems (PVTOS) experiments in which copper phthalocyanine was epitaxially deposited onto highly oriented seed films of metal-free phthalocyanine in low Earth orbit on space shuttle mission STS51 were recently reported. ${ }^{21.22}$ The microgravity-grown copper phthalocyanine films had several desirable features indicating that the growth of metal-organic films in low gravity might result in higher-quality films for NLO applications. For example, results of analysis by photography, interference contrast microscopy, scanning ellipsometry, and visible reflection spectroscopy all suggest that the space-grown films were more uniform and homogeneous than Earth-grown counterparts. Also, as much as an order-of-magnitude improvement in achieving smoother surfaces resulted from the low-gravity PVT of these films over scales of roughness from the submillimeter to the submicron. ${ }^{23}$ Finally, analyses involving the use of external reflection-absorption infrared spectroscopy, grazing incidence $x$-ray diffraction, and visible near-infrared reflection-absorption spectroscopy all indicate that microgravity-grown films are more perfectly textured and, curiously, consist predominantly of polycrystalline domains of a previously unknown polymorphic form of copper phthalocyanine. ${ }^{24}$

\section{RECOMMENDED STUDIES}

Polymeric and organic materials show promise for NLO applications because they offer some flexibility in optical, chemical, and mechanical properties. ${ }^{25}$ One particularly interesting class of efficient NLO compounds is the polydiacetylenes, which include crystalline and polymeric compounds. ${ }^{26}$ These materials also provide the advantage that they can be formed readily as thin films, which is a useful starting configuration for device fabrication. The optical quality of a polydiacetylene film depends critically on the quality and orientation of the monomer layer from which it is obtained. Like the phthalocyanines, the polydiacetylenes are highly conjugated organic compounds with large nonlinear optical responses, benefiting in still not understood ways from microgravity deposition by physical vapor transport.

Solution crystal growth experiments have been performed repeatedly in microgravity, particularly in the protein crystal growth program. Many organic materials, including some with NLO potential, are also amenable to crystal growth from solutions, and some of these offer advantages as subjects of kinetic and dynamic studies of solution growth properties. Ground-based studies of the growth of crystals of a number of important organic materials, including 1 -arginine phosphate and some of the diacetylenes, have been undertaken to assess the effects of gravity-driven convection flows, supersaturation, and temperature on growth kinetics. Given some of the apparent benefits of microgravity in the growth of some protein single crystals, selected examples of important organic materials should also be considered candidates for microgravity studies.

In contrast to inorganic materials, organics and polymers are nearly infinite 
in variety and number. This makes the selection and preparation of specific materials for study both difficult and time consuming, unless there are specific goals and effective methods for accurate screening. In the case of optical performance, several theoretical methods promise to provide strong predictive tools for appraising the potential of organic materials. To approach the predicted theoretical limits established by these calculations, the materials must be defect free. The use of molecular mechanics may also yield theoretical guidance concerning the preferred orientation of molecules within a given film, crystal, or composite, which in turn provides for estimations of second-and third-order nonlinear optical coefficients for such materials. A ground-based research program to screen for such properties may enhance the selection of candidates for NLO materials and the identification of those with the greatest potential for microgravity studies, and greatly reduce the work required to synthesize, process, and test NLO materials both on Earth and in microgravity.

Recently, NASA/MSAD has approved funding for several new basic polymer science-related projects, including three studies dealing with diffusion effects occurring in monomer droplets dispersed in nonsolvent fluids. Specifically, traveling wave front polymerization will be investigated under circumstances where autocatalysis leads to rapid polymerization. Emulsification of monomer droplets during dispersion and suspension polymerization will be studied in microgravity to avoid the difficulties caused by buoyancy-induced coalescence. Also, light scattering and optical interference will be used to monitor bulk copolymerization reactions in which diffusional growth of the polymer chains will be followed under microgravity conditions. Finally. some anomalous viscosity phenomena arising in supersaturated (i.e., metastable) melts will be investigated through a study of precritical fluctuations and "clustering," which occurs both in nonideal solutions and in some complex molecular melts prior to nucleation and growth.

\section{RECOMMENDATIONS AND CONCLUSIONS}

Polymers potentially represent the broadest classes of "engineered" materials, permitting great innovation and precision in their design, including control at the molecular level. Nevertheless, although opportunities exist for microgravity research, relatively little is being done in NASA's current microgravity research program.

- Although the viscous character of most high polymer melts greatly desensitizes their response to gravitational acceleration, initial experiments in some areas of vapor- and solution-phase processing of organic and polymer films in microgravity have shown improved texture and smoothness over terrestrial counterparts, which suggests that this area of research merits further study. 


\section{GROWTH OF INORGANIC SINGLE CRYSTALS}

\section{INTRODUCTION AND GOALS}

Although studies of both metals and alloys, and ceramics, may involve crystalline materials, the objective in their preparation is generally not to prepare individual single crystals. There are a few materials, however, that as single crystals are of enormous technological importance. Pervasive examples are crystals of silicon and of quartz. The former is the most important semiconductor material, and the latter is the major material used for crystal oscillators. In addition, there are several other important semiconductor materials. A variety of single crystals are used for electrooptics, an important technology in solid-state lasers, modulators, and detectors for energetic particles and electromagnetic radiation.

Reasonable goals for studying the growth of inorganic single crystals under microgravity conditions are to contribute to an understanding of the fundamental processes that take place during crystal growth, to provide benchmark crystals of higher quality than can be obtained terrestrially, and/or to provide useful experimental data that cannot be obtained (or obtained as accurately) in terrestrial studies.

Currently, and in the foreseeable future, the most important single-crystal material is silicon ( $\mathrm{Si}$ ). It is used for most solid-state electronics for circuitry that ranges from a myriad of small microprocessors to the processing components of the largest supercomputers. It is the best-studied semiconductor and quite possibly the best-studied inorganic material there is. Among semiconductor materials, those composed of elements from groups III and V of the periodic table are second in importance. These compounds are isoelectronic to silicon, but different enough that their distinctive properties allow them to serve very important niches in photonics and in ultrahigh-speed circuitry. Except for a relatively large market for field-effect transistor circuits fabricated on bulk-grown GaAs, group III-V electronic devices are usually fabricated from complex "heterostructures" composed of very thin layers of crystalline group III-V solid solutions and binary compounds grown epitaxially onto a single-crystal wafer of a binary group III-V compound. The latter is most often GaAs or InP.

Substrate quality or availability is not limiting progress at present in the leading-edge technologies, although most crystal growth is based more on empirical experimentation than on a firm scientific understanding of the entire process. Applications to photonics and high-speed applications are expected to expand in the next decade as fiber optics moves closer to the end consumer and as cellular and other wireless communications technologies become more pervasive and move to higher frequencies. Infrared transmission property improvements are expected in group II-VI compounds (e.g., HgCdTe), which may provide advances in Department of Defense and NASA detector applications. 


\section{SUMMARY OF TERRESTRIAL GROWTH OF INORGANIC SINGLE CRYSTALS}

The commercial growth of single-crystal boules (particularly of semiconductors, and frequently of laser crystals) is conducted primarily by three methods: pulling the growing crystal from the melt (the Czochralski method); the floating zone method; and in the case of compound semiconductors, Bridgman and gradient freeze techniques. For other types of malerials, there are a number of methods, including solution growth and, particularly for some oxides, hydrothermal growth (usually at high temperature and pressure).

The Czochralski method consists essentially of dipping a rotating seed crystal into a large container of molten starting material, molten silicon in the case of silicon crystals or, for the growth of compound semiconductors, a nearly stoichiometric mixture of the components. With the temperature of the seed very close to the melting point and the proper temperature gradients, slow pulling of the seed from the melt results in controlled precipitation on the seed and growth of the single crystal. Although the growing crystal is not in contact with the crucible wall, the melt is, and contamination (e.g., the introduction of oxygen into silicon from silica crucible walls) may limit purity.

In the floating zone method, a small section of a cylindrical boule is melted, usually by induction heating, and the molten region is moved parallel to the axis of the cylinder so that the crystal is regrown as the molten region passes through it. This inherently containerless method, useful for the preparation of silicon with exceptionally low oxygen concentration, requires that surface tension counteract the tendency of the molten zone to flow under gravity. For this reason the molten zone is usually a thin slice whose maximum thickness depends on the diameter of the boule. Floating zone growth under microgravity conditions is not subject to gravity-induced flow, and so large stable molten regions will be limited only by surface tension forces.

The Bridgman and gradient freeze methods involve the imposition of temperature and concentration gradients on a solution containing the components for growth. The solution is saturated only near the growth front. This method (which is employed in both vertical and horizontal growth variations) is used extensively for group III-V and II-VI compounds and is well exemplified by GaAs growth, where the solution is mostly liquid gallium. Arsenic is introduced into the solution as the crystal grows onto the seed by maintaining a partial pressure of $\mathrm{As}_{4}$ (arsenic tetramer molecules) with an external source of heated solid arsenic. As the crystal grows, the overall temperature is lowered so that the growth front moves in the direction of higher temperature in the temperature gradient. Under terrestrial conditions, the growing crystal is in contact with the crucible wall. 


\section{THE INFLUENCE OF GRAVITY}

In these growth methods and variations thereof, temperature and/or concentration gradients in the liquid are imposed to promote growth. There are several ways in which crystalline defects and unwanted impurities, or impurity distributions, can occur in the bulk crystal. Some of these have been addressed in microgravity growth studies. ${ }^{27-30}$

- Defects can form in the crystal after growth. In this case, defect formation is not gravity dependent except for crystals with very weak bonding (soft materials) where flow-induced faults originating in hydrostatic forces occur. Semiconductors, laser crystals, and most crystals used for electrooptics are hard materials, not subject to the latter. However, occasionally, as with $\mathrm{HgI}_{2}$ (used for detectors), gravity-induced stress is thought to lead to defects, perhaps immediately after growth, when the crystal is still hot. For this reason, growth (from the vapor) of $\mathrm{HgI}_{2}$ crystals in orbiting vehicles is being pursued.

- Defects can form as the result of disturbances in the interfacial region and from convection in the melt during growth. In this case, constitutional supercooling in the melt near the interface may be modified by gravity because the associated concentration gradients will involve buoyancy convection. In addition, the conditions at the growing interface are complicated by release of the heat of fusion and by differential diffusion of components near the growth front. All of these result in thermal and density gradients, and thus also involve buoyancy convection at the growing interface and in the bulk of the liquid. Since crystal growth occurs by mass transfer in the region where all of these processes occur, it is strongly affected by gravity unless countermeasures are taken. Convection may also be the result of variation of surface tension with temperature and composition at the fluid-fluid interface such as the free surface of melts (thermocapillary effect). Convection resulting from the thermocapillary effect is independent of gravity, except in that it may be masked by buoyancy convection.

- Defects can form, and unwanted impurities can be incorporated, as a result of the interaction of the crystal or the melt with the container during and after growth. Especially deleterious are crystalline defects formed as a result of the crystal's sticking to the container and as a result of different expansion coefficients between the crystal and the container material. Gravity is not a factor. However, inasmuch as containers could, in some cases, be eliminated under microgravity conditions, this source of impurities and defects could also be eliminated.

- Crystalline defects can result from condensed impurity particles that circulate in the melt and have multiple interactions with the growing interface. In addition, there are "striations," attributable to abrupt variations in impurity concentration, that result from convection causing slight temperature oscillation at the growing surface. The effects of particulates may be expected to be reduced 
for crystals grown under microgravity conditions if the circulation is reduced by eliminating or reducing convective flow. The elimination of convection also can eliminate impurity striations. However, it should be noted that only convection due to buoyancy is potentially eliminated by reducing gravity.

- Buoyancy convection inhibits detailed study of the diffusion of impurities and major components under concentration and temperature gradients (Soret effect) and of thermal diffusion in conventional terrestrial studies. This will be a problem of increasing importance as computational methods for bulk crystal growth improve and as the quality of the available transport data becomes more critical.

\section{SPACE- AND GROUND-BASED STUDIES}

The Czochralski method is inherently unsuited to microgravity studies because its geometry is obviously maintained by the gravitational field. More practical for that purpose are the Bridgman, float zone, and "droplet" methods (described below). It has been demonstrated in some studies of Bridgman and float-zoned $\operatorname{InSb}^{31}$ in uncrewed satellites that in microgravity, buoyancy convection can be eliminated under conditions where there is apparently no thermocapillary convection. Under such a condition of diffusion-controlled growth, the radial impurity distribution is at least partially determined by the interface shape. Indeed, radial impurity grading can be increased or decreased, depending on the nonplanarity of the interface. In the float zone case, there is an oscillatory mode of thermocapillary convection that is independent of gravity and causes growth striations. However, some microgravity ${ }^{32}$ and terrestrial ${ }^{33}$ studies with silicon growth have shown that thermocapillary convection can be reduced or eliminated by the use of liquid surface coatings. Bridgman crystals did not show such striations but had extensive defects due to strain caused by unpredictable contact with the container walls.

Complete elimination of the container has been accomplished under microgravity conditions with "droplet" experiments where a large molten region at the end of a solidified region is crystallized. ${ }^{34}$ This situation would be useful for the study of very pure, low-defect crystals. Indeed, some studies in orbiting vehicles showed that the defect density could be markedly lower than for similar terrestrial growth. During the recent USML-1 flight, crystals of $\mathrm{ZnCdTe}$ were grown ${ }^{35}$ by a modified Bridgman technique in which much of the crystal surface was kept away from the container wall. Etch pit densities on cross sections of the grown crystal were less than $1000 / \mathrm{cm}^{2}$, one to two orders of magnitude better than crystals grown terrestrially. Partial defects at the surface not in contact with the container walls were apparently absent, and there was evidence that the reduction in stress due to relaxation of hydrostatic pressure under the microgravity conditions was partially responsible for the improved material quality. Such studies provide an existence proof of the possibility of generating improved materials and suggest the possibility of generating fundamental information on 
bulk inorganic crystal growth. These and other studies have demonstrated that the microgravity environment is indeed unique and that the effects of convection may, in some cases, be removed. Growth then results only from mass transfer by diffusion. It should be noted that it is well established ${ }^{36,37}$ that to accomplish the goal of suppression of convection in typical semiconductor melts, the steadystate acceleration and $g$-jitter must be very small. Depending on the acceleration vector, a platform that is stable well below the $10^{-6}-g$ range may be required.

The technologically important semiconductors GaAs, InP, and $\mathrm{HgCdTe}$ present serious difficulties for studies in orbiting vehicles because of their high melting points and the requirements for maintaining adequate component partial pressure. Presumably this is the reason that much of the microgravity semiconductor growth in the U.S. program has been with more tractable materials such as InSb and germanium. However, the Scientific Industrial Association "Nauchny Tsentr" of the Electronic Industry Ministry of the former Soviet Union developed a variety of crystal growth systems for use in orbiting vehicles and exhibited large group III-V, II-VI, and IV-VI boules that were grown in Soviet space vehicles. ${ }^{38}$ Although extravagant claims about low cost and high quality were made in the context of a commercial presentation, little supporting evidence was presented at the conference. ${ }^{39}$ Nevertheless, scientists in the former Soviet Union may have a significant body of experience in the growth of semiconductor crystals. Efforts should be made to obtain a detailed understanding of those experiments, that is, the experimental conditions under which they were grown, and of the resulting crystals. If possible, samples should be obtained and subjected to the diagnostic techniques normally used for terrestrially grown samples.

The terrestrial production of high-quality semiconductor crystals on a commercial scale is now done by the pulling, float zone, or Bridgman method with apparatus that permits precise temperature control over very large volumes. One area in which considerations of microgravity may have impinged on commercial practice is in the use of magnetic fields to reduce convection. Since the liquid from which a semiconductor is grown is metallic, convection can be reduced by imposition of a magnetic field during growth of the crystal. This partial simulation of reduced gravity has been used for the growth of $\mathrm{GaAs},{ }^{40} \mathrm{CdTe},{ }^{41}$ $\mathrm{Hg}_{x} \mathrm{Cd}_{1-x} \mathrm{Te}^{42}$ and some silicon, as described below. Hurle ${ }^{43}$ has pointed out that since the Lorentz force is proportional to fluid flow velocity, magnetic fields will not be effective in completely damping convection, so in the limit of virtually zero convection, microgravity studies may become necessary. He suggests that magnetic damping might be useful even in space, to reduce the deleterious effects of $g$-jitter.

All commercially important semiconductor crystals, except possibly for $\mathrm{Hg}_{x} \mathrm{Cd}_{1-3} \mathrm{Te}$ and $\mathrm{CdTe}$, may now be considered commodity items. Silicon boules are now routinely grown defect free, with diameters as large as $20 \mathrm{~cm}$ and a length of 1 meter. Both $\mathrm{GaAs}^{44}$ and $\mathrm{InP}$ are available commercially with a 
diameter of $7.5 \mathrm{~cm}$. Wafers sliced from all of these materials are used as the host material for large-scale production of mass memories and as substrates for epitaxial growth. For the latter, given sufficiently high quality in the substrate, the quality of devices and circuits that result depends primarily on epitaxy and subsequent processing. In the case of silicon, the presence of oxygen as an impurity is very often required to "getter" other unwanted impurities from the epitaxially grown material. In that case, the oxygen in the substrate must be uniformly distributed. Magnetic suppression of convection using a carefully shaped magnetic field is used both to distribute the oxygen uniformly and to help reduce its concentration to acceptable levels. The commercial use of magnetic fields for suppressing or managing convection in both group III-V and silicon growth is apparently at least partially the result of NASA-supported terrestrial studies. Clearly, high substrate quality is a prerequisite for almost any use of semiconductor wafers, and any reduction in defect density will have commercial impact. However, it is doubtful whether defects in the substrate are severely limiting commercial applications at present.

It is important to emphasize that as with the introduction of magnetic fields described above, there is ongoing ground-based research-some of it supported by NASA - that contributes to the understanding of bulk growth and impinges on the field of microgravity crystal growth. The following are three illustrative instances pertinent to microgravity studies: (1) A study ${ }^{45}$ has demonstrated an experimental approach that permits adjustment of temperature gradients in a modified Bridgman method, which yields both steady-state diffusion-controlled growth and axially uniform impurity distribution. The method, which uses a submerged heater, should also largely suppress thermociaillary convection. So far it has been used only for the growth of bismuth, but extension to other materials is obvious. (2) Studies, ${ }^{46}$ with conventional Czochralski growth, of a variety of group III-V solid solutions have shown that by the use of slow growth and large melt volumes, uniform and low-defect boules of these materials can be obtained. (3) A modeling study ${ }^{47}$ has shown that for the growth of $\mathrm{HgCdTe}$ the principal influences on defect density result from interface shape, thermal gradients, heat extraction, and cooling rate. Note that of these, only the interface shape is modified by gravity.

Virtually all crystal growth experiments that have been conducted in orbiting vehicles have been "one-shot" experiments. It seems unlikely that a continuation of this kind of approach will be productive of much useful basic information. Terrestrial crystal growth studies are rarely singular events. Indeed, they usually involve many repetitions with, in the optimum instances, rapid feedback of information on the properties of the grown crystal dictating the choice of new growth parameters and even dictating the modification of the growth apparatus. For some studies, parameter variation during growth, under the control of a trained observer, is necessary. Except for elimination of the thermocapillary effect by the use of liquid coatings under some conditions, much of what has been leamed so far in microgravity studies was predictable. The reduction of strain-induced defects with the 
elimination of containers was unsurprising and certainly not basic. The use of magnetic fields to suppress convection in the liquid-metal melts used in semiconductor growth also did not require low-gravity studies. However, this work illustrates the continuing usefulness of NASA-supported ground-based studies that seek to reduce convection. These have been, and will continue to be, important. Obviously, such studies are attempting to approximate microgravity effects and thus avoid the necessity of going into space.

\section{RECOMMENDATIONS AND CONCLUSIONS}

Studies aimed at obtaining better crystals constitute much of the microgravity work done so far. They do not appear to have contributed significantly to the fundamental understanding of crystal growth or to terrestrial commercial practice. The growth, in space, of low-defect, high-purity crystals by variations of the droplet method, zone melting, the use of partially confined melts, ${ }^{48}$ or vapor transport might provide a limited amount of material for terrestrial study of especially high-quality materials to serve as standards. One instance is a "soft" (a crystal that flows under $\mathrm{l}-\mathrm{g}$ hydrostatic pressure) material such as $\mathrm{HgI}_{2}$. However, for hard materials, particularly semiconductors, there does not seem to be a compelling justification for producing such standards.

- The design and execution of microgravity experiments that lead to a better fundamental understanding of crystal growth have proved elusive, and the committee is recommending against the growth of large-diameter inorganic crystals under low gravity. The best approach to understanding the details of such growth will likely derive from fluid dynamical modeling and the modeling of processes at the fluid-solid interface along with terrestrial studies of crystal growth. This analytical approach may provide the rationale for the growth of benchmark-quality inorganic crystals in microgravity.

- If some of the versatility of terrestrial experimentation can be achieved in the microgravity environment, there are opportunities for microgravity research that will impact terrestrial bulk crystal growth. Priority should be given to transport studies including studies of solute and self-diffusion, heat diffusion, and Soret diffusion. All of these are studies of the fluid from which crystals are grown. They are amenable to routine study with repeatedly used apparatus. However, they may require a more stable environment than provided by the space laboratory. Indeed, since such research will undoubtedly be sensitive to the acceleration environment, it may also be useful for the study of this environment as a variable in the low-gravity range. Any experiments on bulk crystal growth must be judged on their potential for contributions to the scientific understanding of the fundamental processes of crystal growth.

- Precise transport data will become particularly useful since fluid dynamical computational capabilities (for which the data are required) are improving rapidly for terrestrial melt and solution growth, as well as other industrial processes. Fur- 
thermore, transport measurements in industrially important fluids may be an important microgravity application outside the realm of inorganic crystal growth.

\section{GROWTH OF EPITAXIAL LAYERS ON SINGLE-CRYSTAL SUBSTRATES}

\section{EPITAXIAL GROWTH METHODS}

The growth of epitaxial layers of semiconductors on single-crystal wafers, and the controlled incorporation of impurities into those layers, constitute a major thrust of semiconductor crystal growth research at present. These epitaxy methods fall into three primary groups:

- Liquid phase epitaxy (LPE). This method has been used for epitaxy of compound semiconductors and is still used extensively for the growth of layers of garnet. In LPE, the layer precipitates on the substrate from a solution saturated according to the requirements of the phase diagram of the liquid-solid system. For semiconductors, LPE is rapidly decreasing in commercial importance as vapor epitaxy and beam epitaxy methods take over.

- Chemical rapor deposition (CDV) methods. For silicon, CVD involves transport of the silicon as a gaseous halide that decomposes on the substrate surface at elevated temperature. The chloride transport methods for silicon and the group III-V compounds are usually carried out at atmospheric pressure. For silicon epitaxy the process is well controlled and is the standard way that highquality layers are obtained. For group III-V compounds, these methods have produced high-quality growth compounds that do not contain aluminum. However, the relatively high temperature of the process (about $700^{\circ} \mathrm{C}$ ) limits the ability to incorporate impurities at the high concentrations needed for some devices and limits interface abruptness. This is a technology that is well in hand for silicon and is in somewhat decreasing use for group III-V heterostructures.

- Metalloorganic chemical vapor deposition (MOCVD) and molecular heam epitaxy (MBE) methods. These epitaxy methods are of increasing importance for growth of heterostructures of group III-V compounds and appear to be the methods that will predominate in group III-V epitaxy over the next decade. The MOCVD methods are best exemplified for the growth of group III-V binary and solid solution layers. In this case, the method involves passing a gas stream containing $\mathrm{AsH}_{3}$ and/or $\mathrm{PH}_{3}$ plus group III alkyl organics, such as triethylgallium, over a heated group III-V binary substrate in a cold-wall growth system. This is done at either atmospheric or reduced pressure, but always in the viscous flow regime. The starting compounds undergo decomposition reactions both in the boundary layer adjacent to the growing surface and on that surface itself, with resultant epitaxy of a thin single-crystal layer. In MBE, beams of the elements or the same metalloorganic compounds used for MOCVD are projected against the heated substrate under vac- 
uum conditions where all flow is molecular. In that case, all reactions occur on the growing surface.

\section{RECOMMENDATIONS AND CONCLUSIONS}

The issues that are currently of most importance in the exploitation of these techniques are growth quality, uniform thickness, compositional uniformity, and problems relating to homogeneous dopant incorporation. The latter include unintentional doping, doping uniformity, redistribution, and achievable doping range. In addition, a major challenge that is beginning to be addressed is the development of selective area epitaxy methods for group III-V compounds.

With MOCVD methods, there are certainly issues of convection, flow, buoyancy, and boundary layer uniformity, all of which are affected by gravity. Experimentally, an effort is made to minimize these effects by rotating substrates during growth. In addition, efforts are being made to calculate precise flow patterns and resulting growth rates in model systems. ${ }^{49}$ The calculations are complex, and they might not be able to predict the behaviors of real systems that are useful for industrial production. Since the manufacture of heterostructures by MOCVD will be accomplished terrestrially, the impact of research in microgravity is limited. Further, given the complexity of the calculations for that situation, it is not clear that there is relevant information to be obtained through microgravity research.

- Low priority should be given to CVD studies at this time.

As for the MBE methods, microgravity is clearly not an issue. Surface reactions and transport by molecular flow over distances measured in hundreds of millimeters do not involve gravity. However, some workers believe that the use of space as an infinite vacuum makes space epitaxy studies interesting. This might be true if the use of space for manufacture of large areas of epitaxial wafers is economically feasible, which seems very doubtful. Alternatively, a space-based study suitably isolated from contamination (e.g., behind a wake shield) might yield information about epitaxy under conditions of minimal background contamination. For such a study, only small samples would be needed. Here, we must recognize that contamination arises both from the sources and from the vacuum systems in conventional beam epitaxy systems. The former would be present in space studies too. The committee concludes from studies of ultrapure high-mobility GaAs grown by $\mathrm{MBE}^{50}$ that, with sufficient contamination reduction and increased pumping speed, any required great gains in purity can be made with terrestrial studies.

- The committee discards the idea that epitaxial layers will be manufactured in space and notes that, at a reasonable cost, much improvement in the vacuum environment can be achieved terrestrially. Studies in orbiting vehicles are not in order until ground-based alternatives have been thoroughly examined. 


\section{CERAMICS AND GLASSES}

\section{INTRODUCTION}

In a broad sense, a ceramic is any man-made, inorganic, nonmetallic, solid material. A glass is a solid that lacks crystalline order. Traditionally, ceramics have been considered to be polycrystalline, although most ceramists today would not accept that restriction. Also traditional is the idea that high temperatures are required for the synthesis or processing of ceramics and glasses. That limitation also is no longer valid, as evidenced by such new materials as aerogels and tin fluorophosphate glasses that are synthesized at room temperature or at a few hundred degrees above ambient temperature.

Ceramics are predominantly ionically bonded compounds found in complex crystal structures that are strong, stiff, lightweight, hard, and corrosion resistant and that maintain their properties to high temperatures. Ceramics also are brittle, which makes them susceptible to catastrophic failure under mechanical load. The useful strength of a ceramic is determined by the flaw population; stresses are concentrated at flaws, which cause cracks to propagate to failure. The critical property for ceramics in load-bearing applications is not strength but fracture toughness, the resistance of the ceramic to crack propagation. Much of current ceramic processing research is directed at increasing the fracture toughness, either through elimination of flaws or by making the ceramic less sensitive to the flaw population (increasing the critical flaw size or increasing the fracture energy).

Raw materials for ceramic and glass manufacture traditionally are earthy, oxide materials that are mined in high volume at low cost and are subjected to relatively little processing. The products made from them are commodity items such as bricks, tile, and glass windows. Modern technical or engineering ceramics are higher-value-added materials that have superior properties by virtue of their more sophisticated processing and tighter control over raw materials. It is to this latter class of materials that the microgravity environment has the greatest relevance.

\section{CURRENT ISSUES IN CERAMICS RESEARCH}

As discussed in several publications, ${ }^{51-53}$ the greatest needs and opportunities in ceramics lie in the areas of synthesis and processing. Specific recommendations for increased emphasis include:

- Interactive research on new materials synthesis that is linked with characterization and analysis of the product:

- Basic research on synthetic solid-state inorganic chemistry to produce new compounds; 
- Synthesis of ultrapure materials, for example, fibers with low oxygen or carbon impurity levels;

- Research on techniques for synthesis to net-shape, that is, learning how to do synthesis, processing, and forming in a single step;

- Research on methods for processing ceramic materials far from equilibrium; and

- Research on processing of artificially structured or, as they are sometimes called, functionally gradient materials.

Whether the availability of a free-fall environment is relevant to these research areas is an unanswered question.

\section{MICROGRAVITY AS A VARIABLE IN CERAMICS SYNTHESIS AND PROCESSING}

Evaluating the applicability of the microgravity environment to opportunities and problems in ceramics $R \& D$ requires a good definition of that environment. Two aspects of this environment seem relevant to ceramics: (1) the possibility of levitating a specimen for high-temperature containerless processing and (2) the suppression of buoyancy-driven convection. In both cases, the applicability is to gas- or liquid-phase phenomena. Thus, for applications of microgravity to ceramics, one should look to where liquid or gas phases are important. Some areas of liquid-and gas-phase processing of ceramics are discussed below.

\section{Melt Synthesis}

Ceramics are very seldom made directly by melt processing, mainly because their melting temperatures are very high (for those that do not decompose first), the melts react severely with available crucible materials, and undesirable microstructures are obtained on cooling. Thus, ceramics typically are made by sintering of powders. Those ceramic powders are produced by a variety of techniques, including upgrading of mined deposits, solid-state reactions, solid-liquid reactions, and gas-phase reactions. For some powder compositions, such as highpurity $\mathrm{Al}_{2} \mathrm{O}_{3}$ or $\mathrm{SiC}$, sintering and densification are entirely by solid-state processes. For other ceramics, a second-phase liquid may be present in sintering (up to $15 \%$ by volume). The unanswered question is what difference microgravity might make for ceramic melts.

Availability of a general method for contamination-free synthesis using containerless melting would be of value for producing research specimens of new high-temperature materials. Lack of promising new techniques is probably one of the main reasons that melt synthesis of ceramics or inorganic compounds is no longer a very active research area. Ceramic superconductors, such as $\mathrm{YBa}_{2} \mathrm{Cu}_{3} \mathrm{O}_{1}$, have been proposed for levitation melting to minimize crucible 
contamination. ${ }^{54}$ However, here the problem is not reaction with the container but that $\mathrm{YBa}_{2} \mathrm{Cu}_{3} \mathrm{O}$, melts incongruently and on cooling does not give the stoichiometric compound-a problem not addressed by levitation. Many of the benefits of microgravity containerless melting are available on Earth using skull or cold-wall melting. The relative merits of space-based containerless melting and terrestrial cold-wall melting have not been assessed for ceramic synthesis. Vaporization is frequently a problem in ceramic and glass synthesis and processing. For example, $\mathrm{Si}_{3} \mathrm{~N}_{4}$ vaporizes incongruently at temperatures below those required for significant solid-state diffusion. Thus, $\mathrm{Si}_{3} \mathrm{~N}_{4}$ ceramics are made with additives that lower the sintering temperature (to $1700-1800^{\circ} \mathrm{C}$ ) by producing small amounts of liquid phases.

Most commercial glasses are made from high-temperature melts. Reaction with crucible materials is always a concern for glass melting, but it is usually dealt with case by case. Glasses crystallize by a nucleation and growth mechanism. In some cases the crystallization is desired, as with glass-ceramics. For glasses, however, crystallization is undesirable and nucleation must be suppressed. Heterogeneous nucleation occurs at phase interfaces, as in the meltcrucible wall or on impurities or heterogeneities in the melt. Containerless melting could be advantageous for some glasses.

Glass melts frequently experience preferential loss of the more volatile components, changing the composition of the resulting glass. Processing in a convection-free environment could lead to higher levels of saturation in the atmosphere at the solid or liquid surface that would suppress evaporation. Lower rates of evaporation would expand the available processing windows for many materials and allow shorter processing times.

Optical fibers are the application of glasses that has the most stringent requirements for purity and homogeneity. Optical fibers are pulled from preforms made by a CVD process and are not melt synthesized. Their manufacture is already highly developed and fibers with optical losses less than $1 \mathrm{~dB} / \mathrm{km}$ in lengths of $30 \mathrm{~km}$ are routinely drawn in a single pull.55 New materials for optical fibers and signal amplifiers are under development. It is conceivable that containerless processing could be useful for producing research specimens of some materials.

\section{Solution Synthesis}

A recent trend in ceramic synthesis is use of near-room-temperature solution techniques to synthesize ceramic precursors. Increasingly, advanced ceramics are synthesized from highly processed, chemically prepared powders. Some of the more promising routes to advanced ceramic powders are sol-gel processing, precipitation from solution, gas-phase synthesis, and powder-surface modification. The sol-gel method is a familiar example. In that technique, organometallic reagents in solution are hydrolyzed and condensed to form an 
inorganic polymeric gel that, when dried and fired, gives the desired ceramic composition. These chemical methods can generate controlled size distributions, extremely reactive precursors, unusually shaped particles, and gels. Solution methods permit intimate mixing of components, easy dispersion of second phases, and surface modification of the precursor particles. Liquid precursor solutions also can be used to make thin films by dipping or spinning, and because of the high reactivity of the precursor particles, film consolidation occurs at moderate temperatures. This advantage is being exploited in research on the synthesis of electronic ceramic films such as PZT (lead zirconate-lead titanate) and $\mathrm{YBa}_{2} \mathrm{Cu}_{3} \mathrm{O}_{7-1}$ from solution. One potential application might be in epitaxial growth of films from solution. That is one example of biomimetic synthesis of ceramics, a proposed approach to ceramic synthesis that mimics biological processes through the use of self-assembling monolayers. Whether reduction of convective transport would be useful to biomimetic synthesis should be determined experimentally.

Chemical preparation of ceramics is a broad subject. For example, synthesis of ceramic precursors by polymerization of metal alkoxides using hydrolysis and condensation reactions is a rapidly expanding area of ceramics. Ultrafine ceramic particles with enhanced surface reactivity (such as $\mathrm{SiO}_{2}$ ) can be synthesized through nucleation or condensation reactions in gas-phase aerosols. Ceramic powders can be prepared under hydrothermal conditions in which the solubilities of many oxides are greatly enhanced, permitting direct precipitation of oxides from solution that is not possible under ambient temperature and pressure. $\mathrm{Hy}$ drothermal growth is well established for growth of quartz crystals, but the full potential of the technique has not been exploited.

Glass, which is also considered a ceramic, has a lack of crystallinity as its most distinguishing feature. Glass precursors can be synthesized in the same ways as ceramics, for example, the process for making the silica boules from which optical fiber is pulled:

$$
\mathrm{SiCl}_{4}+\mathrm{O}_{2}\left(\mathrm{C}_{2} \mathrm{H}_{2} \text { flame }\right) \rightarrow \mathrm{SiO}_{2}+\mathrm{CO}_{2}+\mathrm{H}_{2} \mathrm{O}+\mathrm{HCl}
$$

Glasses can also be made by using the alkoxide solution route described above for sol-gel ceramics.

\section{Powder Synthesis in Microgravity}

One of the main objectives in ceramic powder synthesis is to make particles that are fine, homogeneous, and unagglomerated. Preparation by precipitation from solution requires rapid introduction and mixing of reagents. One typical method, used for electronic ceramics such as $\mathrm{ZnO}$ varistors and $\mathrm{YBa}_{2} \mathrm{Cu}_{3} \mathrm{O}_{4}$, is to pump the solutions into an ultrasonic mixing cell where reaction occurs on the order of micro- to milliseconds and the products are carried away to be removed by filtration. The microgravity environment does not naturally provide any of 
those conditions. Microgravity would seem most obviously of benefit in situations where convection and particle settling must be minimized.

In traditional powder synthesis, the amount of liquid is a small fraction of the total mass, and capillary effects should be much larger than those due to convection. Ordinary gas-phase synthesis techniques involve forced flow of the gases, and it is not clear how large an effect is introduced by convection. It is possible to design experiments with gas flow rates low enough so that convection is relatively important. The significant practical problem in gas-phase synthesis is avoiding agglomeration of very fine particles. Understanding nucleation and growth of those particles is an importan basic science issue.

In summary, gravity may be a significant variable in solution synthesis of ceramics, but that has yet to be demonstrated. When ceramic powder is the goal, reactions normally are run very quickly in a flow reactor or with stirring to obtain a very fine, homogeneous precipitate. Forced mixing is the rule, and any convection is a very minor effect. In sol-gel synthesis of bulk objects such as aerogels, convection may play a more important role, and some gelation experiments in microgravity may be fruitful.

\section{CERAMIC AND GLASS PROCESSING IN MICROGRAVITY}

Processing refers to the steps involved in going from the precursor powder to the final object. There can be a great deal of overlap between synthesis and processing because some schemes are more or less continuous from raw material to final product.

As already discussed, much traditional ceramic processing is by sintering of powders. Melt processing of ceramics is rare, being used mostly for glasses and single-crystal growth (e.g., ruby laser crystals). There are several practical reasons for this, among them the fact that the melting points of many important ceramics are very high (greater than $2000^{\circ} \mathrm{C}$ for $\mathrm{Al}_{2} \mathrm{O}_{3}$ ) and ceramic melts react with many crucible materials. Other ceramics, such as $\mathrm{SiC}$ and $\mathrm{Si}_{3} \mathrm{~N}_{4}$, cannot be melt processed because they melt incongruently. Where reaction with crucible materials is the limiting factor, containerless processing in microgravity may have significant advantages.

There might be opportunities for microgravity research in solution processing of ceramics. One example is making sol-gel films by dip coating. Use of ceramics as films is a rapidly expanding area with applications as diverse as sensors, microelectronic memory elements, and antireflection coatings. It is important to determine the physical and chemical factors that control film formation during dip and spin coating and to develop strategies to tailor film porosity and microstructure for sensor, membrane, protective, and photonic applications. A major issue is the balance between gravitational draining, solvent evaporation, 
solute particle interactions, and surface tension effects. A key to the latter is the hydrostatic capillary pressure, which has rarely been measured in a film or gel. ${ }^{50}$

Solution deposition is of interest for preparing other kinds of ceramic thin films because of the low capital investment costs, the ability to closely control composition, and the relative ease of process integration with other technologies. One problem with the use of chemical deposition methods to prepare thin films is lack of a fundamental understanding of the effects of solution chemistry variations and structural evolution on film properties. For example, the process variables used in each step of the deposition of PZT ferroelectric thin films (e.g. solution preparation conditions, heat treatment temperatures, times, and ramp rates) determine the ferroelectric properties of the film. Reduction of convectivi flows in the microgravity environment may be of benefit in the preparation of high-quality ceramic films, particularly where epitaxy is desired. This would be especially useful if it led to improved processing on Earth.

\section{BASIC SCIENCE}

There are a few fundamental issues in ceramics, in addition to those already mentioned, for which the microgravity environment might prove useful. Perhaps the most obvious is the study of crystal nucleation and growth in glass melts. This question is important technically because of its relation to glassceramics (where one wants controlled crystallization) and to optical glasses (where crystallization is to be avoided). This line of research is being pursued in the NASA containerless melt program. ${ }^{57}$ There is a large body of work in the scientific literature from the 1960 s and early 1970 s on glass crystallization. ${ }^{5 \times}$ The challenge to microgravity research is to show how microgravity can be used for critical experiments that will provide new advances in our understanding of crystal nucleation and growth.

A second fundamental research opportunity is mass transport or diffusion studies in glass and ceramic melts. For many systems of interest, the data are inaccurate or unknown. Diffusion studies in microgravity would not be troubled by convective mixing, and better data could be obtained in much less time. One area of application is using diffusion data to test theories that classify glasses as either strong or fragile, a concept that is related to the degree of polymerization in the melt.

\section{RECOMMENDATIONS AND CONCLUSIONS}

The above discussion identifies a number of areas in which a microgravity environment might be of benefit to ceramics research and development. The following is a prioritized listing of those points:

1. The development of a general method for contamination-free synthesis 
using containerless melting would provide a capability not available on Earth. Examples are containerless melting of glasses to suppress heterogeneous nucleation and containerless processing to produce research specimens of new glasses for optoelectronic applications.

2. A fundamental study of crystal nucleation and growth in glass melts in microgravity could provide information difficult to obtain on Earth.

3. Mass transport and diffusion studies of glass and ceramic melts under microgravity conditions should generate more precise data than the data available from terrestrial measurements.

4. The suppression of free evaporation from melt surfaces could allow synthesis at higher temperatures than can be done on Earth.

5. The epitaxial growth of films from solution, including biomimetic synthesis (self-assembling monolayers) of ceramics, should be studied.

\section{REFERENCES}

1. Glicksman. M.E., and S.P. Marsh. 1993. The dendrite. Pp. 1107-1112 in Handbook of Crystal Growth, Vol. Ib, D.J.T. Hurle, ed. Elsevier, Amsterdam.

2. Johnston. M.H., and R.A. Parr. 1982. Met. Trans., 13B:85.

3. Glicksman, M.E., and M. Koss. 1994. Dendritic growth velocities in microgravity. Phys, Rev. Lett., 73(4):573-576.

4. Pirich, Ron G., and D.J. Larson. 1982. Materials processing in the reduced gravity environment of space. Materials Research Society Symposium Proceedings. 9:523-531.

5. Lacy, L.L., and G.H. Otto. 1975. AIAA Journal, 13:219.

6. Grugel. R.N., T.A. Lograsso, and A. Hellawell. 1982. Directional solidification of alloys in systems containing a liquid miscibility gap. Pp. 553-561 in Materials Processing in the Reduced Gravity Environment of Space. Elsevier Science Publishing Co., Inc.

7. Andrews, J.B., A.L. Schmale, and A.C. Sandlin. 1992. J. Cryst. Growth, 119:152-159.

8 Hayes, J.L., and J.B. Andrews. 1994. Experimental methods for microgravity materials science. R.A. Schiffman and J.B. Andrews, eds. TMS, The Minerals, Metals and Materials Society Proceedings, 6:167-174.

9. Margrave. John L. 1982. Heat capacities of liquid metals above 1500 K. Pp. 39-42 in Materials Processing in the Reduced Gravity Environment of Space. Elsevier Science Publishing Co., Inc.

10. National Aeronautics and Space Administration. 1989. NASA Laser Light Scattering Advanced Technology Development Workshop-1988. NASA Conference Publication 10033.

11. Vanderhoff. J.W.. ct al. 1983. Preparation of large particle size monodisperse latexes in microgravity, P. 17 in Materiah Sciences Under Microgravity, Abutracts of the 4 th European Symposium, Madrid, Spain.

12. Micale F.J.. J.W. Vanderhoff, and R.S. Snyder. 1977. Analysis of electrophoresis on Apollo 16. Separation and Purification Methods, 6:1-59.

13. Owen, R.B. 1980. Optical measurements and tests performed in a low gravity environment. Society of Photo-Optical Instrumentation Engineers Journal, 255:74-81.

14. Riley, C.. D. Coble. and G. Maybec. 1987. Electrodeposition of melals and metal/cermet composites in low gravity. AIAA Paper 87-0510. American Institute for Aeronaulics and Astronautics, Washington, D.C.

15. Dorsinville. R.. et al. 1989. Optical Letters, 14(23):1321. 
16. Kajzar, F., and J. Messier. 1986. Resonance enhancement in cubic susceptibility of Langmuir-Blodgett multi-layers of polydiacetylene. Thin Solid Films, 132:11.

17. Ho. Z.Z., C.Y. Ju, and W.M. Hetherington III. 1987. 3rd harmonic-generation in phthalocyanines. J. Appl. Phys, 62:716.

18. Debe, M.K., R.J. Poirier. D. Erickson, T.N. Tommet, D.R. Field, and K.M. White. 1990. Effect of gravity on copper phthalocyanine thin films. Thin-Solid-Films-Switzerland 186:257-288.

19. Debe, M.K., and K.K. Kam. 1990. Effect of gravity on copper phthalocyanine thin films. Thin-Solid-Films-Switzerland, 186:289-325.

20. Debe, M.K., and R.J. Poirier. 1990. Effect of gravity on copper phthalocyanine thin films. Thin-Solid-Films-Switzerlind, 186:327-347.

21. Debe, M.K., E.L. Cook, R.J. Poirier, L.R. Miller, G.J. Follett, and S.M. Spiering. 1987. Polymer Preprints, 28(2):453.

22. Debe, M.K. 1986. Industrial materials processing experiments on board the space shutule orbiter. J. Vac. Sci. Technol, A4(3):273.

23. Debe, M.K., E.L. Cook, R.J. Poirier, L.R. Miller, G.J. Folletl, and S.M. Spiering. 1987. Polymer Preprints, 28(2):453.

24. Debe. M.K. 1986. Industrial materials processing experiments on board the space shuttle orbiter. J. Vac. Sci. Technol., A4(3):273.

25. National Aeronautics and Space Administration. n.d. Research and Technology Operating Plan (RTOP): Study of Organic Nonlinear Optical Thin Films and Bulk Crystals. Chemistry and Polymeric Materials Branch, Marshall Space Flight Center, Ala.

26. Heeger, A.J., and D.R. Ulrich, eds. 1988. Nonlinear optical properties of polymers. Symposium Proceedings Ser., Vol. 109. North Holland Press. New York.

27. Witt, A.F., H.C. Gatos, M. Lichtensteiger, and C.J. Herman. 1978. Crystal-growth and segregation under zero gravity-Ge. J. Electrochem. Soc., 125:1832.

28. Witt, A.F., H.C. Gatos, M. Lichtensteiger. M.C. Lavine, and C.J. Herman. 1978. Crystal growth and segregation under zero gravity: germanium. J. Electrochem. Soc. 125:276.

29. European Space Agency. 1991. Summary Review of Sounding Rocket Experiments in Fluid Science and Materials Science. O. Minster. ESA-1132. February.

30. Mathiesen, D.H. 1991. Growth of electronic materials in microgravity. P. 111 in Proceedings of the Microgravity Science Symposium, Moscow. May 13-19. American Institute of Aeronautics and Astronautics. Washington, D.C.

31. Zemskov, V.S., M.R. Raukhman, E.A. Kozitsina, I.V. Barmin, and A.S. Senchenkov. Experiments on directional crystallization of indium antimonide on 'FOTON' automatic satellites. P. 124 in Proceedings of the Microgravity Science Symposium. Moscow, May 13-19. American Institute of Aeronautics and Astronautics, Washington, D.C.

32. Croell, A., W. Muller, and R. Nitsche. 1986. Floating-zone growth of surface-coated silicon under microgravity. J. Cryst. Growth, 79:65.

33. Eyer, A., and H. Leiste. 1985. Striation-free silicon crystals by float-zoning with surfacecoated melt. J. Cryst. Growth, 71:249.

34. Walter, H.U. 1974. Seeded, containerless solidification of indium antimonide. P. 257 in Proceedings of the 3rd Space Processing Symposium. NASA TMX-70252.

35. Larson, D.J. 1993. Orbital processing of ZnCdTe. Presented at the 14th International Astronautical Congress, Graz, Austriat.

36. Iwan, J., D. Alexander, J. Ouazzani, and F. Rosenberger, 1989. J. Cryst. Growth, 97:285.

37. Alexander, J.I.D., and C.A. Lundquist. 1988. AIAA Journal, 26:193.

38. USSR Scientific Industrial Association, Electronic Industry Ministry. 1991. AIAA Microgravity Science Symposium, Moscow.

39. Private communication. F. Lemkey.

40. Kawase, T., A. Kawasaki, and K. Tada. 1986. Gallium arsenide and related compounds. Inst. Phys. Conf. Ser., 82:27. 
41. Price, M.W., R.N. Andrews, C.H. Su, S.L. Lehoczky, and F.R. Sofran. 1994. The effect of a transverse magnetic field on the microstructure of directionally solidified CdTe. J. Cryst. Growth, 1.37:201-207.

42. Su, Ching-Hua, S.L. Lehoczky, and F.R. Sofran. 1991. Directional solidification of $\mathrm{HgCdTe}$ and HgZnTe in a transverse magnetic field. J. Cryst. Growth, 109:392-4(K).

43. Hurle, D.T.J. 1992. Gravity related phenomena in materials science. Presented at the European International Space Year Conference, Munich.

44. Nambu. K., R. Nakai, M. Yokogawa, K. Matsumoto, K. Koe, and K. Tada. 1987. Gallium arsenide and related compounds. Inst. Phys. Conf. Ser., 91:141.

45. Ostrogorsky, A.G., F. Mosel, and M.T. Schmidt. 1991. Diffusion controlled distribution of solute in $\mathrm{SN}$ - I-percent-BI specimens solidified by the submerged heater method. J. Cryst. Growth. 110:950.

46. Bonner, W., B.J. Skromme, E. Berry, H.L. Gilchrist, and R.E. Nahory. 1988. Bulk singlecrystal Gal-1 In As-..LEC growth and characterization. Inst. Phys. Conf. Ser., 96:337-342, and private communication.

47. Larson, D.J.. Jr., A. Levy, D. Gilles, J.I.D. Alexander, and F.M. Carlson. 1991. Proceedings of the SPIT International Symposium on Optical Science and Engineering, Vol. 1557.

48. Lagowski, J., H.C. Gatus, and F.P. Dabkowski. 1985. Partially confined configuration for the growth of semiconductor crystals from the melt in zero-gravity environment. J. Cryst. Growth, $72: 595$.

49. Jensen, K., D.I. Fotiadis, and T.J. Mountziaris. 1991. Detailed models of the MOCVD process. J. Cryst. Growth, 107:1.

50. Pfeiffer, L., K.W. West, H.L. Stormer, and K.W. Baldwin. 1989. Electron mobilities exceeding $107 \mathrm{~cm}^{2} / \mathrm{s}$ in modulation-doped GaAs. Appl. Phys. Let1. 55:1888.

51. National Research Council. 1989. Materials Science and Engincering for the 1990s: Maintaining Competitiveness in the Age of Materials. National Academy Press. Washington, D.C.

52. Evans, J.W., and L.C. de Jonge, eds. 1991. The Production of Inorganic Materials. Macmillan Publishing Company. New York.

53. ASM. 1991. Ceramics and Glasses, Vol. 4, Engineered Materials Handbook. ASM International, Metals Park. Ohio.

54. Hofmeister, W.H. et al. 1992. P. 47 in NASA Technical Memorandum 4349.

55. MacChesney, J.B., and D.J. DiGiovanni. 1990. Malerials development of optical fiber. J. Am. Ceram. Soc., 73(12):3537-35.59.

56. Brinker, C.J. A.J. Hurd, G.C. Frye, and C.S. Ashley. 1991. Fundamentals of sol-gel dip coating. Thin Solid Films, 201:97-98.

57. The references to Michael Weinberg's work in NASA Technical Memorindum 4349 (February 1992) summarize much of what has been done.

58. Simmons. J.H.. D.R. Uhlmann, and G.H. Beall, eds. 1982. Nucleation and crystallization in glasses. Advances in Ceramics. Vol. 4. American Ceramic Society. Columbus. Ohio. 


\section{7 \\ Microgravity Physics}

\section{INTRODUCTION}

The intersection between the special research possibilities offered by microgravity conditions and the general interest of the physics community is relatively small. This is understandable since gravitational forces play only the most minor role in the traditional areas of physics such as high-energy, nuclear, and condensed matter physics. Nevertheless, there are a number of problems of interest in which access to space and the microgravity environment may allow important research advances.

The subject of microgravity physics consists of many diverse topics united by their common requirement for free-fall conditions. A similar situation holds for low-temperature physics where the subject is united by the technical requirements for low temperatures. The topics of microgravity physics may be cast into three categories for the purposes of discussion.

- New' instruments. The very low stress conditions possible in the microgravity environment allow the construction of instruments of unique sensitivity. Examples are the superconducting gyroscopes of the Gravity Probe-B experiment and the mass balance of Everitt and Worden's equivalence principle test.' Access to space allows the construction of instruments on a length scale not limited by the size of the Earth, such as a long-baseline $\left(10^{6} \mathrm{~km}\right)$ gravitational wave antenna. The freedom from the disturbance of microseisms that set the noise floor for Earth-bound gravitational wave detectors is an important benefit of the microgravity environment.

- Unique samples. Under free-fall conditions, gravitationally induced compression effects are essentially absent and it is possible to create experimental 
samples with unprecedented uniformity. This possibility is particularly important in the area of critical phenomena. Relief from gravitational and vibrational stress will also be important for the preparation and study of delicate structures such as low-density granular materials near the percolation limit.

- New dynamics in fluids. Fluid systems will provide a rich area for the study of new dynamic phenomena that can be expected in the microgravity environment. The absence of buoyancy forces arising from nonuniform density distributions under free-fall conditions allows weaker forces to come into play and produce new dynamic behavior. Understanding the behavior of fluids in space is important not only from the point of view of fundamental science but also for the solution of practical engineering questions involved in spacecraft design. Fluids under microgravity conditions constitute an extensive subject that is discussed separately in this report.

\section{THE CURRENT MICROGRAVITY PHYSICS PROGRAM}

Two extensive reviews of the field of fundamental science in space ${ }^{2,3}$ discuss in considerable depth experiments currently being developed for flight in the near future and longer-term prospects for the field. It is useful to discuss a number of representative experiments to gain an appreciation of the motivations, promise, and difficulties involved in microgravity physics.

\section{Fundamental Physics Measurements}

Access to space and the microgravity environment will allow tests of several of the most fundamental aspects of physics. The most elemental of these is an experiment being developed at Stanford University ${ }^{4}$ to test the equivalence principle. This principle, which asserts the identity of inertial and gravitational mass or the impossibility of distinguishing within a system between acceleration and a gravitational field, lies at the heart of Einstein's theory of gravitation. The equivalence principle is so well imbedded in physics today that one tends to forget that tests of this principle have a long and honored history dating back to the experiments with falling bodies conducted by Galileo. In the 1960s, the equivalence principal was confirmed ${ }^{5}$ to 3 parts in $10^{11}$. The new experiment ${ }^{6}$ promises an eventual improvement on those results by some six or seven orders of magnitude. In the original version of the experiment, the acceleration due to Earth's motion in its orbit was compared to the gravitational attraction of the Sun, whereas the Stanford experiment will compare the gravitational attraction of Earth on an orbiting test mass to the acceleration associated with that orbit. The gravitational force on an object in low Earth orbit is three orders of magnitude greater than the attraction due to the Sun; hence, an immediate large gain in sensitivity is realized by going to space. A second advantage of the microgravity environment is the escape from microseismic disturbances that would limit an 
Earth-bound experiment. As currently developed, this experiment takes advantage of modern cryogenic technology with superconducting bearings for the confinement of the test mass and SQUID (Superconducting Quantum Interference Device) position detectors. A shuttle flight for this experiment would allow an important test of the operation of this apparatus and should allow an improvement in the test of the equivalence principle resolution to the level of 1 part in $10^{15}$. Given a successful shuttle flight, there would be a strong motivation to fly this experiment on an independent satellite where the microgravity environment could be substantially improved and the full promise of this experiment might be realized.

A second experiment developed by the Stanford group is the very ambitious attempt to observe the "frame-dragging" or magnetogravitational effects predicted by Einstein's general theory of relativity. This experiment, which has been under development for more than 20 years, is known as Gravity Probe-B (GPB). It consists of four gyroscopes for sensing the frame-dragging effects that will appear as extremely small torques on the gyros. The spin axes of the gyros will then be compared with a fixed star. This experiment makes heavy use of advanced cryogenic technology with the utilization of superconducting gyros and superconducting readouts. The time scale envisioned for this experiment is on the order of a year. Although GPB can be given a shakedown test in a shuttle flight, an independent satellite will be required to satisfy the microgravity requirements of the experiment, which lie in the $10^{-10}-\mathrm{g}$ range.

\section{Critical Phenomena}

The study of critical phenomena has become prominent in condensed matter physics over the last 20 years. This period has seen remarkable advances on both the experimental and the theoretical fronts, in particular with the highresolution experiments at the 1-point transition of liquid ${ }^{4} \mathrm{He}$ and with the development and successful application of renormalization group techniques to the calculation of the behavior of many systems in the neighborhood of their critical transitions.

The subject of critical phenomena presents a very promising area for microgravity research. The essential advantage of microgravity for studies of critical phenomena lies in the possibility of achieving relief from the gravity-induced compression effects always present in the Earth-bound laboratory. These compression effects are particularly severe in liquid-vapor critical point studies because the compressibility of the system diverges as the critical point is approached. Near the transition, the gradient in the hydrostatic pressure leads to a large density gradient in the sample. Thus, only a diminishing portion of the sample will satisfy the condition for critical density as the transition is approached. The situation with the superfluid transition in liquid ${ }^{4} \mathrm{He}$ is somewhat more favorable since the order parameter does not couple strongly to the density. 
Nevertheless, on Earth, gravity-induced hydrostatic pressure leads to a microkelvin shift in transition temperature for each centimeter of sample height. Gravity effects can be minimized, to some degree, by a reduction in the height of the sample, but this strategy soon runs into sensitivity problems related to sample size, and on a more fundamental level, problems associated with surface and finite-size effects set limits to sample thickness.

The $\lambda$-point in liquid helium has been a paradigm for critical phenomena studies. Liquid helium offers a number of properties that make it an almost ideal subject for such studies. ${ }^{78}$ It is an exceptionally pure system. In the superfluid state, helium possesses a very rapid relaxation time, and compared to the liquidvapor transition the superfluid transition is relatively insensitive to gravitational effects. The use of samples less than $1 \mathrm{~mm}$ thick has allowed useful data to be obtained at reduced temperatures, $\mathrm{t}=1-\mathrm{T} / \mathrm{T}_{\lambda}$, less than $10^{-7}$. Modern advances in low-temperature thermometry would allow at least a two-order-of-magnitude improvement in experimental resolution.

Motivated by the central position of the superfluid $\lambda$-point transition in the critical phenomena field, Lipa and Chui at Stanford University have developed a microgravity version of the $\lambda$-point heat capacity experiment. ${ }^{9}$ In the development of this experiment, they have advanced the state of the art in high-resolution thermometry to the point that they can resolve temperature to 1 part in $10^{11}$. Microgravity conditions are required to fully exploit this superb resolution. In space, the heat capacity measurements can be extended two orders of magnitude to the $10^{-9}$ level in reduced temperature beyond the best results achieved in ground-based measurements.

The Lambda Point Experiment (LPE) was flown on the shuttle in October 1992 (USMP-1). Analysis of the data indicates an improvement of nearly two orders of magnitude over previous data obtained on Earth. Unfortunately, the final analysis was delayed by several factors. The first was the failure of NASA to provide the investigators with the full data set on an expeditious time scale. At least four months passed after the touchdown of USMP-1 before a complete record of the raw experimental data became available for analysis. A second factor was the discovery, as is often the case during innovative research, of an unanticipated experimental problem. The cosmic-ray background encountered in orbit was found to produce stochastic thermal spikes in the response of the high-resolution thermometers, resulting in a somewhat degraded performance. The principal investigator has developed an algorithm for combing the data for the worst of these spikes. This was done in an unbiased way, and heat capacity data approaching within a few nanokelvin of the $\lambda$-point have been obtained.

The LPE has been an outstanding success. This experiment gives a clear demonstration that highly sophisticated experiments involving the most sensitive and advanced instrumentation can be performed profitably in the microgravity environment.

A second critical point experiment is also in an advanced state of prepara- 
tion. This is the light scattering experiment developed for measurement of the correlation length at the liquid-vapor critical point of xenon. ${ }^{10}$ This experiment should be able to test the theory of density fluctuations in the nonhydrodynamic limit much closer to the transition than would be possible on Earth. It has been expected that the phenomenon of critical slowing (i.e., the divergence of the time scale for thermal equilibrium as the transition is approached) will be an important experimental constraint. This would be especially true for an experiment conducted on the shuttle, where there will be stringent time constraints. There are indications, however, that there may be an actual speeding up of equilibrium near the transition." If true, then the feasibility of liquid-vapor critical point experiments will be much enhanced.

\section{FUTURE EXPERIMENTS UNDER MICROGRAVITY CONDITIONS}

Critical phenomena, particularly studies at low temperature, have occupied a central position in the current program of microgravity research. This is due to the advanced level of experimental and theoretical sophistication achieved in this area of condensed matter physics as a consequence of the vigorous progress of the last two decades. It is expected that the future will see continued experimental advances from the marriage of modern high-resolution measurement techniques with the vastly improved sample uniformity possible under microgravity conditions.

New directions are possible for the study of critical phenomena in space. Up to this time the emphasis has been on measurement of static properties, particularly the specific heat in the neighborhood of the critical transition. For the future, several new directions are possible that represent important extensions beyond the successful completion of LPE. Chief among these will be studies of systems far from equilibrium, nonequilibrium dynamic phenomena, and studies of finite size effects in well-characterized geometry. Again, liquid helium will be the system of choice, given the large body of experience and its experimental advantages. Enthusiasm for further work with liquid helium in space will be strongly enhanced by success with LPE.

Although low-temperature critical phenomena experiments are the most advanced and tend to drive the field, there are many possibilities for studies of critical phenomena in other systems that can benefit from microgravity conditions. Wetting phenomena, nucleation and growth, and the critical behavior in microemulsions and polymer systems, as well as the behavior of fractal aggregate systems near their percolation threshold, hold considerable potential for microgravity research.

Beyond critical phenomena, there are interesting studies to be made on the static and dynamical properties of low-density aggregate structures and suspensions. Freedom from sedimentation effects will be most important in such work. 
One interesting aspect of low-density aggregate structures is their fractal nature. On Earth, there is a minimum to the density of an aggregate structure that can be stable since such structures tend to collapse of their own weight, because their strength decreases more rapidly with decreasing density than does the gravitational stress.

Atom trapping and laser cooling techniques have been advanced in recent years to the point where atomic transitions can be studied under conditions of free-fall. In "atomic fountain" experiments, groups of ultracold atoms are projected upward into a microwave cavity. The dwell time for these atoms, under free-fall conditions, is limited by the size of the cavity and Earth's gravitational field. Current experimental configurations allow observation times as long as $0.5 \mathrm{~s}$ and should allow the development of an atomic clock with the remarkable accuracy of 1 part in $10^{16}$. This type of experiment is a clear candidate for the microgravity environment, where the experimental observation time could be greatly extended with a further major increase in clock accuracy.

The dynamics of crystal growth is another subject that can profit from microgravity conditions. To date, there has been considerable interest in the possibility of improved crystal size and quality with growth under microgravity conditions where convection is suppressed. Another aspect to the crystal growth problem is the influence of gravity on the facet size and ultimate equilibrium shape of the crystal. These questions could probably be best addressed through studies of growth of free-floating helium crystals under microgravity conditions. ${ }^{12-14}$ Helium crystals are particularly good candidates for microgravity research because they can be rapidly grown, melted, and annealed. Surface relaxation is so rapid that melting-freezing waves are easily created. The roughening transition, readily observed in helium crystals, makes their shape strongly influenced by gravity. ${ }^{15}$ A large reduction in gravitational influences would facilitate studies of this unique type of phase transition.

\section{RECOMMENDATIONS AND CONCLUSIONS}

The experiments mentioned above, with the exception of the GPB, all represent extensions of work that is currently performed or can be performed in Earthbased laboratories. Much of the scientific value of the proposed space experiments will depend on the strength of the connection to Earth-bound research. Given the long time scale for the development through flight of a space experiment, there is a real danger that the scientific goals of the experiment may be bypassed by new developments or by major shifts in the value ascribed to the work. There is also the possibility that the principal investigator (PI) may lose contact with the field. This is particularly likely if the PI becomes heavily involved with the development of flight hardware for a long period of time. These considerations argue for considerable support of ground-based research in the same general area as the candidate 
flight experiments. Also, it is desirable to keep approved projects under periodic scientific review throughout the life of the experiment.

The quality of the microgravity environment is a topic that must be examined critically in the context of each possible experiment. Minimum acceleration is the most obvious parameter of concern for many of the contemplated experiments; however, the time span over which a high-quality, low-gravity environment can be maintained may be of equal importance. For some experiments, accidental large accelerations, such as might result from the sudden movement of personnel or the firing of small thrustors, may destroy the object of study, for example, a low-density granular structure.

When considering the quality of the microgravity environment available for experiment, one should remember that only the center of the orbiting spacecraft is in true free-fall and then only to the extent that orbital drag effects and other external influences such as solar wind and radiation are negligible. In a gravity gradient-stabilized spacecraft, there will be a steady rotation of any experiment about the center of mass of the entire spacecraft once each orbit. For a low Earth orbit, this will result in accelerations on the $10^{-7} \mathrm{~g}$ level at a distance 1 meter from the center of mass of the entire orbiting system. On both the space shuttle and the space station only a few experiments will be located close enough to the center of mass to ensure acceleration below the $10^{-6}$ level.

- It can be expected that a number of scientifically meritorious projects, such as the equivalence principle experiment and GPB, will require spaceflight independent of the crewed space facilities.

In the future, one may anticipate a continued requirement for low-temperature facilities in space since low temperatures are important for the highest-resolution measurement techniques, particularly those based on SQUID technology.

- If the space station is to be a useful contributor to the area of fundamental science, access to liquid-helium facilities will be mandatory.

\section{REFERENCES}

1. Hazelrigg, G.A., and J.M. Reynolds, eds. 1986. Opportunities for Academic Research in a Low-Gravity Environment. Progress in Astronautics and Aeronautics, Vol. 108. American Institute of Aeronautics and Astronautics, New York.

2. Hazelrigg, G.A., and J.M. Reynolds, eds. 1986. Opportunities for Academic Research in a Low-Gravity Environment. Progress in Astronautics and Aeronautics, Vol. 108. American Institute of Aeronautics and Astronautics, New York.

3. National Research Council. 1988. Space Science in the Twenty-First Century: Imperatives for the Decades 1995 to 2015. Fundamental Physics and Chemistry. Nattonal Academy Press, Washington, D.C.

4. Hazelrigg. G.A., and J. M. Reynolds, eds. 1986. Opportunities for Academic Research in a 
Low-Gravity Environment. Progress in Astronautics and Aeronautics, Vol. 108. American Institute of Aeronautics and Astronautics, New York.

5. Roll, P.G., R. Krotkov, and R.H. Dicke. 1964. Ann. Phys., 26:442

6. Hazelrigg, G.A., and J.M. Reynolds, eds. 1986. Opportunities for Academic Research in a Low-Gravity Environment. Progress in Astronautics and Aeronautics, Vol. 108. American Institute of Aeronautics and Astronautics, New York.

7. Ahlers, G. 1976. Physics of Liquid and Solid Helium, Vol. I. K.H. Bennemann and J.B. Ketterson, eds. John Wiley \& Sons, New York.

8. Wilson, K,G, 1971. Renormalization group and critical phenomena. 1. Renormalization group and the Kadanoff scaling picture. Phys. Rev. B, 21:3174-3183.

9. Hazelrigg, G.A., and J.M. Reynolds, eds. 1986. Opportunities for Academic Research in a Low-Gravity Environment. Progress in Astronautics and Aeronautics. Vol. 108. American Institute of Aeronautics and Astronautics, New York.

10. Bourkari. H., M.E. Briggs, J.N Shaumeyer, and R.W. Gammon. 1990. Phys. Rev. Lett., $65: 2654$.

11. Bourkari, H., M.E. Briggs, J.N. Shaumeyer, and R.W. Gammon. 1990. Phys. Rev. Lett.. 65:2654.

12. Andreev, A.F., and A.Y. Parshin. 1978. Sov. Phys. JETP, 48:776.

13. Keshishev, K.O., A.Y. Parshin, and A. Babkin. 1979. Experimental detection of crystallization waves in HeSUP(4). Sov. Phys. JETP Lett., 30(1):56-59.

14. Lipson, S.G., and E. Polturak. 1987. The surface of helium cryslals. Pp. 127-188 in Progress in Low Temperature Physics, Vol. XI. D.F. Brewer, ed. North-Holland. Amsterdam.

15. Keshishev, K.O.. and A.Y. Parshin. 1979. Sov. Phys. JETP Letl., 12:56. 


\section{PART III}

\section{Programmatic Issues}





\section{8 \\ Flight Opportunities and Challenges}

\section{INTRODUCTION}

The microgravity research program began in the late 1960s, primarily as a microgravity materials processing activity. It was initially an outgrowth of the space race between the Soviet Union and the United States and was concerned not just with scientific endeavors but also with the commercial materials marketplace. Early experiments on Apollo, Skylab, and Apollo-Soyuz provided the foundation for a research field that is now nearly a quarter of a century old. In the 1970s, the credibility of the program suffered somewhat when efforts failed to demonstrate the near-term rewards promised by enthusiasts. Europeans, meanwhile, focused their program on microgravity fluid and materials science issues using drop towers, parabolic flights, sounding rockets, and high-altitude balloons. The Soviets took advantage of a nearly continuous presence in space with flight experiments in microgravity environments. During the late 1980s and early 1990s, the United States strengthened its programs in microgravity research with numerous shuttle flights, leading to a plan to utilize a space station as a long-term, permanently crewed facility.

In developing a program of microgravity research, NASA has attempted to provide a balance of opportunities for its various constituencies: industry, government, and academia. Academia has traditionally focused on fundamental research, industry on commercial applications, and NASA on the mission frequency and ground-based infrastructure to support reasonable research progress. With the reduction of flight opportunities following the Space Shuttle Challenger accident, NASA recognized that a reliable spaceflight infrastructure was essential if U.S. industry and academia were to expand their participation in the

\section{1}

$119,1 \geq 0$

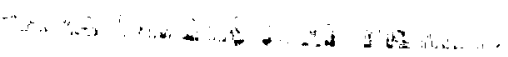


program and provide the next generation of space scientists. Providing a reliable infrastructure, however, has greatly increased the complexity of flight experiments and, consequently, increased the costs of shuttle missions. Together with the development and utilization of sophisticated technologies in optics, electronics, computers, communication devices, and sensors, dedicated missions in particular have become more costly to develop and operate.

In an attempt to keep costs to a minimum, NASA constructed a number of multiuser spaceflight instruments and hardware for several microgravity scientific and technological communities. These instruments, identified through a complex internal evaluation and comparison process, have not proved as useful to a wide spectrum of investigations as intended.

Because microgravity research is a laboratory science, it often requires realtime interactions between scientists and their experiments. Moreover, a laboratory-based researcher must be able to carry out a large number of experiments over ranges of experimental parameters and demonstrate the reproducibility of results. When necessary, the researcher must be able to modify the apparatus and employ diagnostics to achieve these goals.

NASA's microgravity research program must, therefore, be sufficiently broad and robust to encompass requirements for basic research as well as provide the foundation for development of the many processes leading to future space-based technologies. The program of support should be designed to be flexible so that it can rapidly accommodate new areas of investigation or scientific interest as they develop.

To achieve greater flexibility in terms of instruments. procedures, and approaches that should be introduced into the program, a number of special administrative points should be considered:

- Access to the microgravity environment should be increased substantially; this means, for most practical purposes, access to space shuttle missions and ultimately to a long-duration orbiting platform such as the space station. However, while the value and need for human intervention capabilities are noted repeatedly in this report, nothing herein should be interpreted as advocating or opposing any one of several initiatives or programs, such as a space station, planned by NASA in coming years.

- Hardware versatility, variety, and flight manifests should be made more flexible so that research development builds on flight experience in a systematic and expedient manner.

- Some microgravity experiments can benefit from an investment in teleoperational capability, allowing scientific investigators direct interaction with their experiments. NASA should encourage and support the development and deployment of such techniques in future microgravity experiments.

- The cost of flight instruments for research in microgravity should be substantially reduced by lessening bureaucratic overhead, minimizing technical complexity, and eliminating unnecessary requirements. Approaches must be 
devised to obtain initial feasibility data and proof of concept from simplified. economical devices before any commitment is made to the long-term development of expensive instrument systems.

- As much as possible, instruments should be shared among divisions, particularly between the Life Sciences and the Microgravity Science and Applications Divisions, and between NASA and the space agencies of other countries, such as the European Space Agency. This could serve to broaden the base of available flight instruments, reduce expense, and encourage a closer cooperation among space scientists.

- Transient accelerations of spacecraft due to equipment operation and crew movement can affect microgravity experiments. In certain experimental situations, the effects of these accelerations and, in particular, the acceptable maximum magnitude are quantitatively well understood. In other cases, however, the effects of these accelerations are only qualitatively known and the maximum acceptable level cannot confidently be prescribed. Research is needed to characterize the effects of such accelerations.

- Some attention should be given to the moderation of safety requirements for experiments in space. The level of containment currently required is often unnecessary, adds needless complexity and overhead, and appreciably increases costs. In addition, it limits flexibility, slows development rates, and places an encumbrance on instrument development that weighs heavily on the overall program. The same is true of the level of toxicological analysis, the time required to effect minor and otherwise insignificant changes in an experiment, and the excessive amount of documentation required to achieve flight qualification. Although any, or all, of the more stringent requirements might be entirely appropriate in specific instances, they should not be broadly and indiscriminately applied.

Another issue relates to the assignment of administrative responsibility in the areas of microgravity effects on cellular and subcellular processes and mechanisms, investigations into microgravity effects on macromolecular assemblies, their responses to physiological stimuli, and the biochemical processes that underlie all of these phenomena. A clear concept and description of the overall scope of the Microgravity Science and Applications Division research effort in the area of biotechnology should be developed. Some mechanism should be found to establish and maintain close cooperation and coordination among investigators in the area and between the Life Sciences Division and the Microgravity Science and Applications Division.

\section{OPPORTUNITIES: MICROGRAVITY RESEARCH FACILITIES}

A variety of facilities are available for research in low-gravity environments. These range from drop towers and aircraft, used to conduct tests that need only short-duration milli- to microgravity conditions, to the space shuttle 
TABLE 8.I Research Facilities for Microgravity

\begin{tabular}{|c|c|c|c|}
\hline Facility & $\begin{array}{l}\text { Gravity } \\
\text { Environment }\end{array}$ & $\begin{array}{l}\text { Duration } \\
\text { of Environment }\end{array}$ & Comments \\
\hline \multicolumn{4}{|l|}{ 5.18-s Zero-Gravity } \\
\hline Research Facility & $<10^{-5} g$ & $5.18 \mathrm{~s}$ & Operational early 1993 \\
\hline $2.2-\mathrm{s}$ drop tower & $<10^{-5} g$ & $2.2 \mathrm{~s}$ & \\
\hline $105-m$ drop tube & $10^{-6} s$ & $4.6 \mathrm{~s}$ & High-temperature capacity \\
\hline$K C+1.35$ aircraft & $\begin{array}{l}10^{-2} g \\
10^{-3} g\end{array}$ & $\begin{array}{l}15-25 s \\
5-15 s\end{array}$ & Free-fall within cabin \\
\hline Learjet & $10^{-1}-10^{-2} g$ & $18-22 s$ & \\
\hline Sounding rockets & $<10^{-5} g$ & Up to $15 \mathrm{~min}$ & \\
\hline Space shuttle & $\begin{array}{l}10^{-6}-10^{-5} g \\
\text { (quiescent period) } \\
10^{-3} g \\
\text { (active period) }\end{array}$ & Variable & \\
\hline Space station & $\begin{array}{l}<10^{-6} \mathrm{~g}, f \leq 0.1 \mathrm{~Hz} \\
<10^{-3} \mathrm{~g}, f \geq 100 \mathrm{~Hz} \\
f \times 10^{-5} \mathrm{~g} \\
0.1 \leq f \leq 100 \mathrm{~Hz}\end{array}$ & $\begin{array}{l}\text { 30 days continuous. } \\
180 \text { days/year }\end{array}$ & $\begin{array}{l}\text { Additional specifications } \\
\text { for complex disturbances }\end{array}$ \\
\hline Free-flyers & $\begin{array}{l}<10^{-5} g, f<1 \mathrm{~Hz} \\
<10^{-3} g, f>100 \mathrm{~Hz} \\
f \times 10^{-5} g . \\
1<f<10 \%) \mathrm{Hz}\end{array}$ & Several months & \\
\hline Lunar base & $0.16 z$ & & \\
\hline
\end{tabular}

and space station. The characteristics of each facility in the NASA program are described below and summarized in Table 8.1. Except where a specific document is cited, the technical specifications of the facilities were obtained through interactions with NASA staff.

\section{Drop Towers and Drop Tubes}

The drop tower or drop tube low-gravity facility relies on free-fall of the experimental package to produce a microgravity environment. Efforts are made to eliminate the retarding effect of the atmosphere. either by evacuating the region in which the free-fall takes place, by use of a drag shield, or by gas thrusters. Typically, microgravity conditions last for only a few seconds, and the experimental package must withstand extreme deceleration at the end of a test. 


\subsection{8-Second Zero-Gravity Research Facility}

The 5.18-Second Zero-Gravity Research Facility, which consists of a tube embedded in the ground that can be evacuated to approximately $10^{-2}$ torr, permits $5.18 \mathrm{~s}$ of microgravity conditions of $<10^{-5} \mathrm{~g}$. The height of the vacuum chamber is $145 \mathrm{~m}$ and allows a free-fall of $132 \mathrm{~m}$. One to two tests can be run per day. This facility is located at the NASA-Lewis Research Center.

\section{2-Second Drop Tower}

The 2.2-Second Drop Tower provides $24 \mathrm{~m}$ of free-fall. Tests are run in air with a drag shield, which leads to a gravitational acceleration of $<10^{-5} \mathrm{~g}$ during free-fall. Up to 12 tests per day can be run at the facility, which also is located at NASA-Lewis.

\section{5-Meter Drop Tube}

The 105-Meter Drop Tube can be evacuated to a pressure of $10^{-6}$ torr, which allows $4.6 \mathrm{~s}$ of free-fall with accelerations that may be as low as $10^{-6} \mathrm{~g}$ (currently not measured). The drop tube is designed such that a sample can be melted and then allowed to solidify during the free-fall to study containerless processing. This facility is at the NASA Marshall Space Flight Center. Up to 45 drops per day are possible.

\section{Parabolic-Flight Aircraft}

Microgravity research aircraft obtain weightlessness by flying a parabolic trajectory. Although large forces of acceleration and deceleration are produced during portions of the flight, in the short period of parabolic flight, conditions of less than $1 \%$ of Earth gravity are obtained. Such periods of weightlessness may be useful for conducting low-gravity experiments requiring somewhat longer times than are available in the drop towers and for those cases in which the quality of the microgravity environment is not critical. Parabolic flights are useful for testing experimental packages intended for later flight on the space shuttle or space station.

\section{KC-135 Aircraft}

When the KC-135 flies through the top of a parabolic flight path, some 15 to $25 \mathrm{~s}$ of $10^{-2}-\mathrm{g}$ low-gravity conditions occur, with 5 to $15 \mathrm{~s}$ of this at the level of $10^{-3} \mathrm{~g}$ for experiment packages that are in free-fall within the cabin. Some 40 trajectories can be flown during a single flight. The KC-135 operates from the Johnson Space Center in Texas and the Lewis Research Center in Ohio. 
Learjet

Low-gravity conditions are maintained for 18 to $22 \mathrm{~s}$ in the Learjet. Typically the acceleration is of the order of $10^{-1}$ to $10^{-2} \mathrm{~g}$ during this period, but the trajectory can be altered to produce intermediate accelerations. Six trajectories can be flown during a single flight. NASA recently acquired a DC-9 that is expected to replace the Learjet sometime in 1995. The capabilities of the DC-9 are similar to those of the $\mathrm{KC}-135$.

\section{Sounding Rockets}

High-altitude suborbital rockets (sounding rockets) have been used extensively for space science research since the end of World War II. In recent years, they have been used for microgravity research. At the top of the arc of the trajectory, a gravitational environment of $<10^{-5} \mathrm{~g}$ exists for up to $15 \mathrm{~min}$. Operational launch vehicles permit payloads up to $420 \mathrm{~kg}$. Recovery systems allow soft impact retrieval of payloads. High data-rate telemetry, real-time ground uplink command, and down-link video data are routinely available.

\section{Space Shuttle}

The operating parameters on the shuttle have been measured by the Space Acceleration Measurement System (SAMS) and the Orbital Acceleration Research Experiment (OARE) to be $10^{-6}$ to $10^{-5} \mathrm{~g}$ for quasi-steady (i.e., low-frequency) accelerations. Disturbances of the order of $10^{-3} \mathrm{~g}$ during on-orbit maneuvers or crew operations are controllable to some extent. Flights are scheduled to last a number of days, but uninterrupted quiet times are brief. Figure 8.1 shows the capabilities of the accelerometer systems, SAMS and OARE, along with typical shuttle acceleration spectra. The standard spacelab double rack available to experimenters is limited to about $634 \mathrm{~kg}$, with $1.75 \mathrm{~m}^{3}$ in volume available for a payload unit. The possibility exists. however, of connecting several units for a given experiment.

The times required for experiments in some of the subdisciplines of microgravity research exceed those available on the typical spacelab mission. Spacelab missions with extended-duration capability might be better able to serve the research communities of those subdisciplines. For others such as biotechnology and materials science, longer flight times would provide significant benefits in terms of the quality of scientific yields. Even for those microgravity experiments that do not require long duration in orbit, the limited number of spacelab flights severely limits reproducibility. These limitations, inherent to the spacelab system, can be obviated only by the longer flight durations available on a space station or free-flyer. 


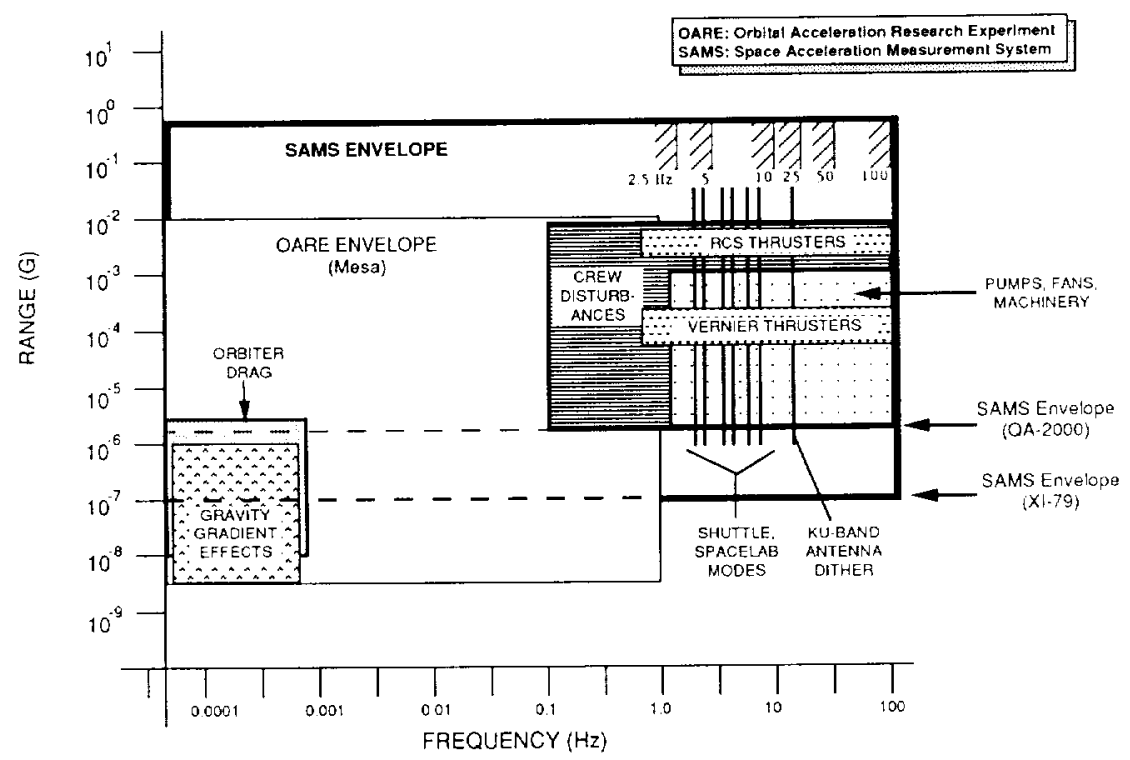

FIGURE 8.1 Accelerometer systems (SAMS, OARE) and typical disturbances on the shuttle.

\section{Space Station}

The following describes the requirements for microgravity research aboard a space station. While the information given here was taken from "Space Station Freedom (SSF) Program Definition and Requirements Document," Revision L (1992), the microgravity requirements remain essentially unchanged in the more recent "Space Station Concept of Operations and Utilization," Vol. 1, Appendix C, Revision A (March 23, 1994). Although the space station concept continues to be redefined, the desired microgravity characteristics are likely to be the same as indicated herein. In specifying allowable accelerations, quasi-steady state means accelerations with frequencies $<0.01 \mathrm{~Hz}$. In this frequency regime, an upper limit of $10^{-6} g$ is imposed on the magnitude of the instantaneous acceleration, and an upper limit of $0.2 \times 10^{-6} \mathrm{~g}$ on the magnitude of the component of the instantaneous acceleration perpendicular to the direction of the average orbital acceleration. (The critical frequency $0.01 \mathrm{~Hz}$ was chosen as being one order of magnitude below a reasonable estimate of the fundamental structural vibration frequency.)

Figure 8.2 shows the allowable accelerations above $0.01 \mathrm{~Hz}$. Considered individually, the total root-mean-square contribution to the acceleration spectrum from 

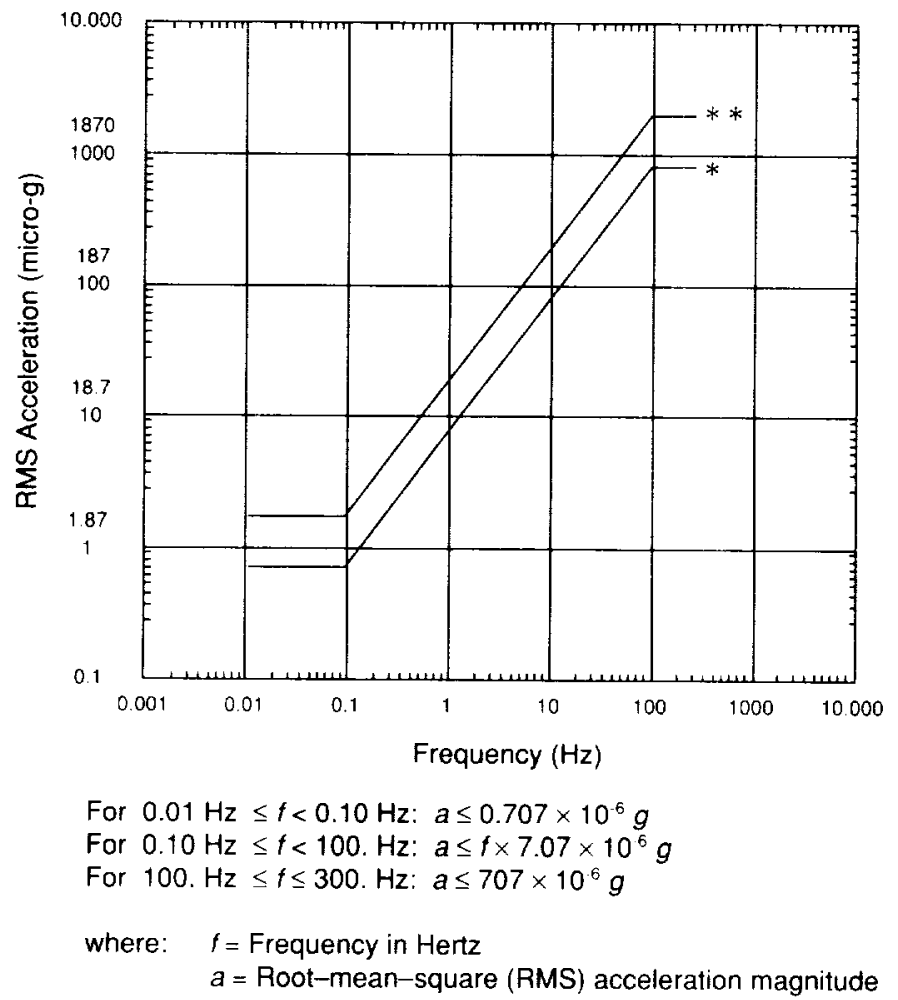

FIGURE 8.2 Space Station Freedom combined sources microgravity allocations. Graph shows allocation limits for each work package, each international partner, and the combined payload complement, at module-to-rack interface. Limits applicable in any onethird octave band from 0.01 to $300 \mathrm{~Hz}$, over any 100 -s interval.

* Individual allocations for three work packages, three international partners, and payloads.

** RMS total of all seven individual allocations. RMS, root mean square.

each work package and from each international partner must not exceed the limits indicated in Figure 8.2. Furthermore, the vibrations induced by the combined payloads must not exceed the root-mean-square acceleration magnitude limits of Figure 8.2. These limits are applicable in any one-third octave band from 0.01 through $300 \mathrm{~Hz}$, over any 100-s interval. Crew interfaces with the space station structure, such as treadmill, keyboards, and drawers, are covered by the vibration budget seen in Figure 8.2. Currently, however, astronaut motion is not subject to the vibration restrictions. Clearly, there is little point in designing the structure and instrumentation of a space station to provide a microgravity environment if the movement of the astronauts introduces unacceptable disturbances. The question of astronaut motion is still under consideration. 
The requirements of Figure 8.2 refer to monochromatic loading with timeindependent amplitudes. Specifications have been written for transient effects. Each individual transient is limited to $10^{-3} \mathrm{~g}$ along each of three mutually perpendicular axes. In any 10 -second window, the time-integrated acceleration must be less than $10 \mu \mathrm{g}-\mathrm{s}$ along each of the three directions. This criterion is applied to each disturbance source separately. In the case of multiple sources, if these are monochromatic with the same frequency the accelerations are summed (with phase relations taken into account) and the result is compared with Figure 8.2. Procedures have been formulated to take into account broad-band disturbances. An example of the analysis of the disturbance to the microgravity environment to be expected from a particular facility is given in Figure 8.3 A.B. Both the narrow-band $(\mathrm{A})$ and the broad-band $(\mathrm{B})$ requirements for the centrifuge facility (CFP) previously planned for Space Station Freedom are shown. Also shown are the "allocations" to the CFP, $35 \%$ of the force required to produce a $10^{-6}-g$ acceleration. The frequency dependence of these curves reflects the frequency response of the structure of the SSF to disturbances. This example is presented for the purposes of illustration only, since more recent space station plans do not yet specify allocations for the centrifuge facility.

Design and operational plans should be such that the microgravity environment is of sufficient quality (including full consideration of transients, multiple sources, and resonant frequencies) and time duration to meet the requirements of the scientific experiments.

\section{Free-Flyers}

Free-flyer data were taken from an Announcement of Opportunity for EURECA- $I$ experiments. The data sheet specified a microgravity environment of $10^{-5} \mathrm{~g}$ for frequencies of $<1 \mathrm{~Hz}$ and $10^{-3} \mathrm{~g}$ for frequencies $>100 \mathrm{~Hz}$. The EURECA environment has been verified by on-orbit measurements. Total payload mass was approximately $1000 \mathrm{~kg}$. The mission was launched in July 1992 and flew for about 10 months.

\section{Lunar Base}

Experiments conducted on the Moon's surface would take advantage of an order-of-magnitude reduction in the gravitational acceleration to $0.16 \mathrm{~g}$.

\section{CHALLENGES TO MICROGRAVITY RESEARCH EXPERIMENTATION}

The present microgravity research infrastructure does not readily accommodate the needs of laboratory-type research. Drop tower experiments and precursor experiments on airplanes flying parabolic trajectories are limited to 


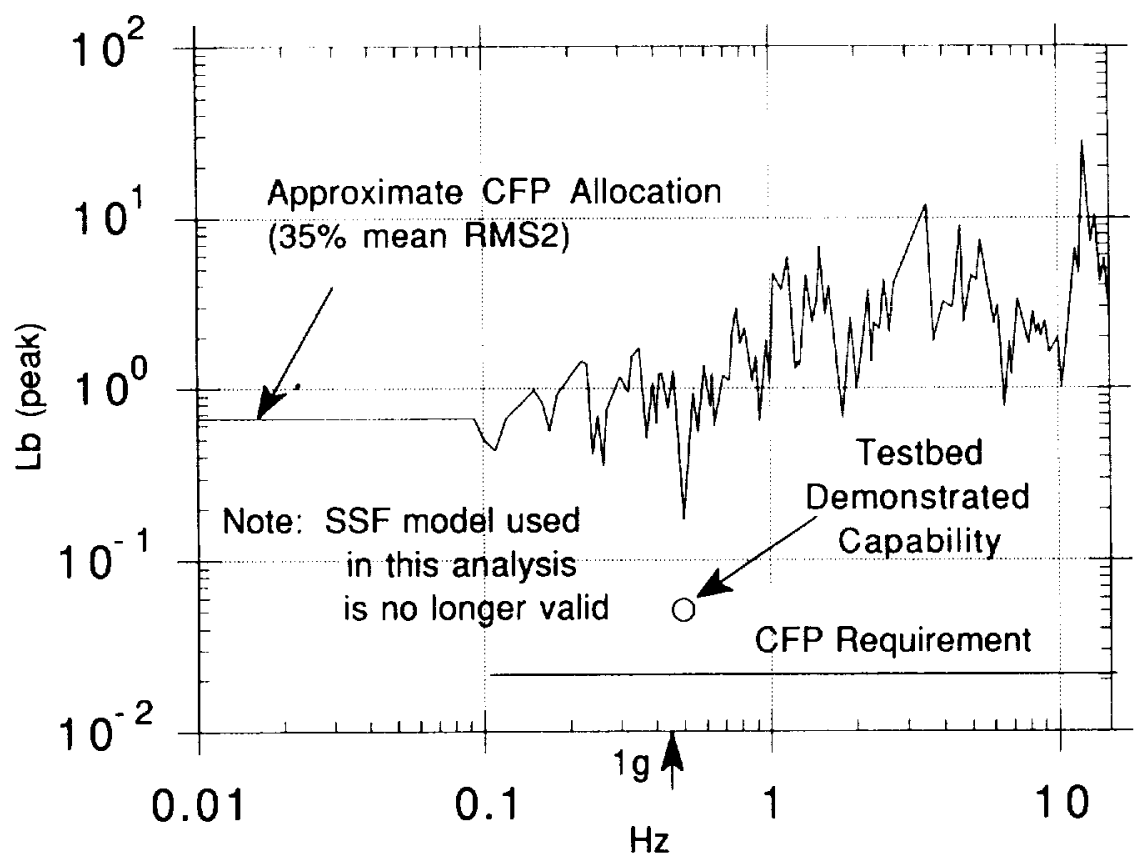

FIGURE 8.3A Approximate Space Station Freedom (SSF) imposed narrow-band force requirement on the centrifuge facility (CFP). RMS, root mean square.

short durations. Robotic materials processing and telescience experiments can be done on free-flyers. Spacelabs and a space station are required for conducting laboratory-type microgravity research. Experience with spacelab for microgravity research, however, indicates that (1) it is likely to take years to develop an experiment that can yield high-quality scientific data and (2) it is an extremely expensive process.

At present, the time required from the selection of a new principal investigator to launch of his or her spaceflight experiment is 5 to 6 years for the middeck (noninteractive) location and 6 to 8 years in spacelab. Reflights with minor modifications to the equipment require a minimum of 1 year for mid-deck and 2 years for spacelab experiments. Every element of an experiment must be documented in detail and pass safety tests. Figures 8.4 and 8.5 outline the process, including numerous extensive reviews at various stages of the development and integration of the experiment. For example, there are 5 reviews in the experiment definition and development process and 11 reviews (including 4 for safety) in the mission integration process. The experiment is required to be delivered a full year before launch for integration into the spacelab and orbiter. 


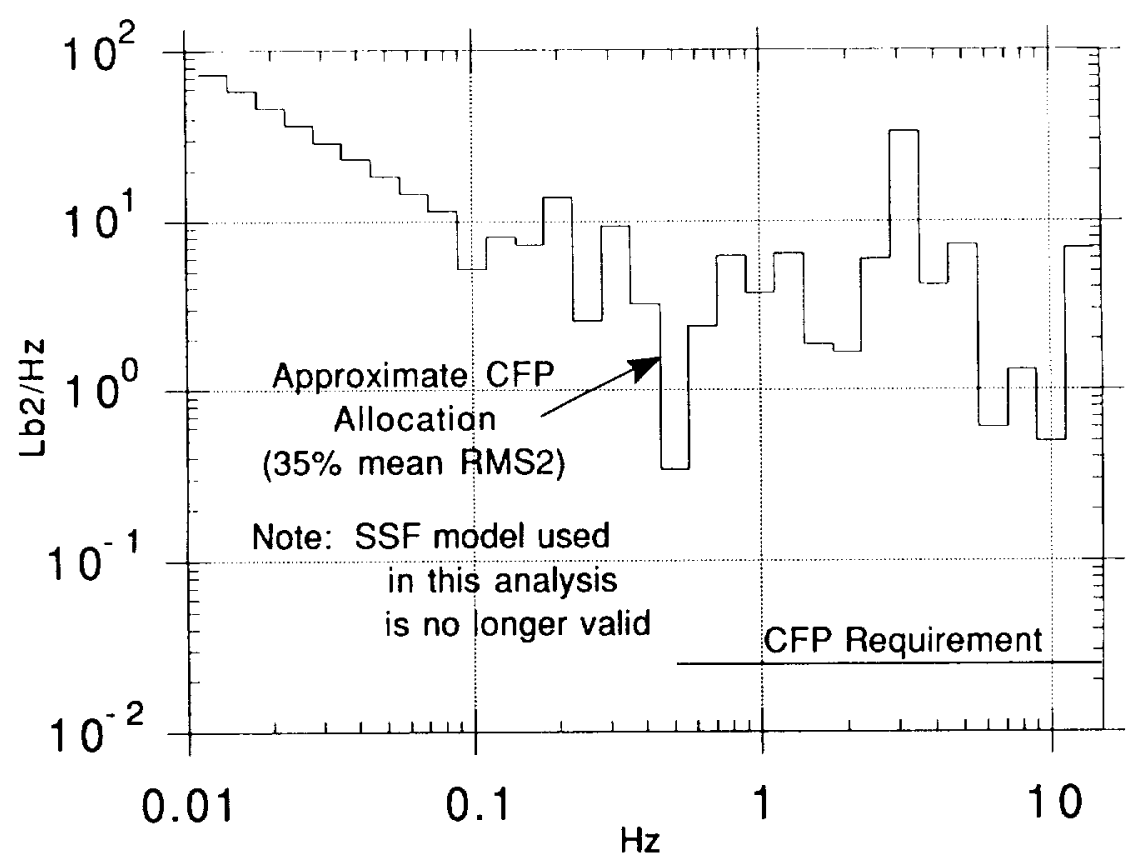

FIGURE 8.3B Approximate Space Station Freedom (SSF) imposed broad-band requirement on the centrifuge facility (CFP). RMS, Root mean square.

Because the number of spacelab missions is limited, as many experiments as possible are scheduled for each flight. Thus, the number of experimental runs is limited even if all goes as planned on the mission. As a result, compromises in the form of flight rules often have to be made in the acceleration environment due, for example, to the orbiter orientation. These flight rules are often favorable for one experiment but not another. Because of heavy demand, experimenters are guaranteed only one flight and any reflights must compete again.

Surmounting the various hurdles to which a flight experiment is subject requires interactions with three different NASA centers: Johnson Space Flight Center, which is in control of the orbiter; Marshall Space Flight Center, which controls the spacelab; and Kennedy Space Center, which is in charge of integration and launch. The conditions imposed on the experiment and the principal investigator by the various NASA centers are not always consistent. In addition, there are many people involved that have sharply delineated areas of responsibility, including systems, safety, quality control, time line, and orbital mechanics. All of this complexity is a result of the concern for the safety of the crew and spacecraft and results in long times for experiment development. 


\section{LIFT OFFI}

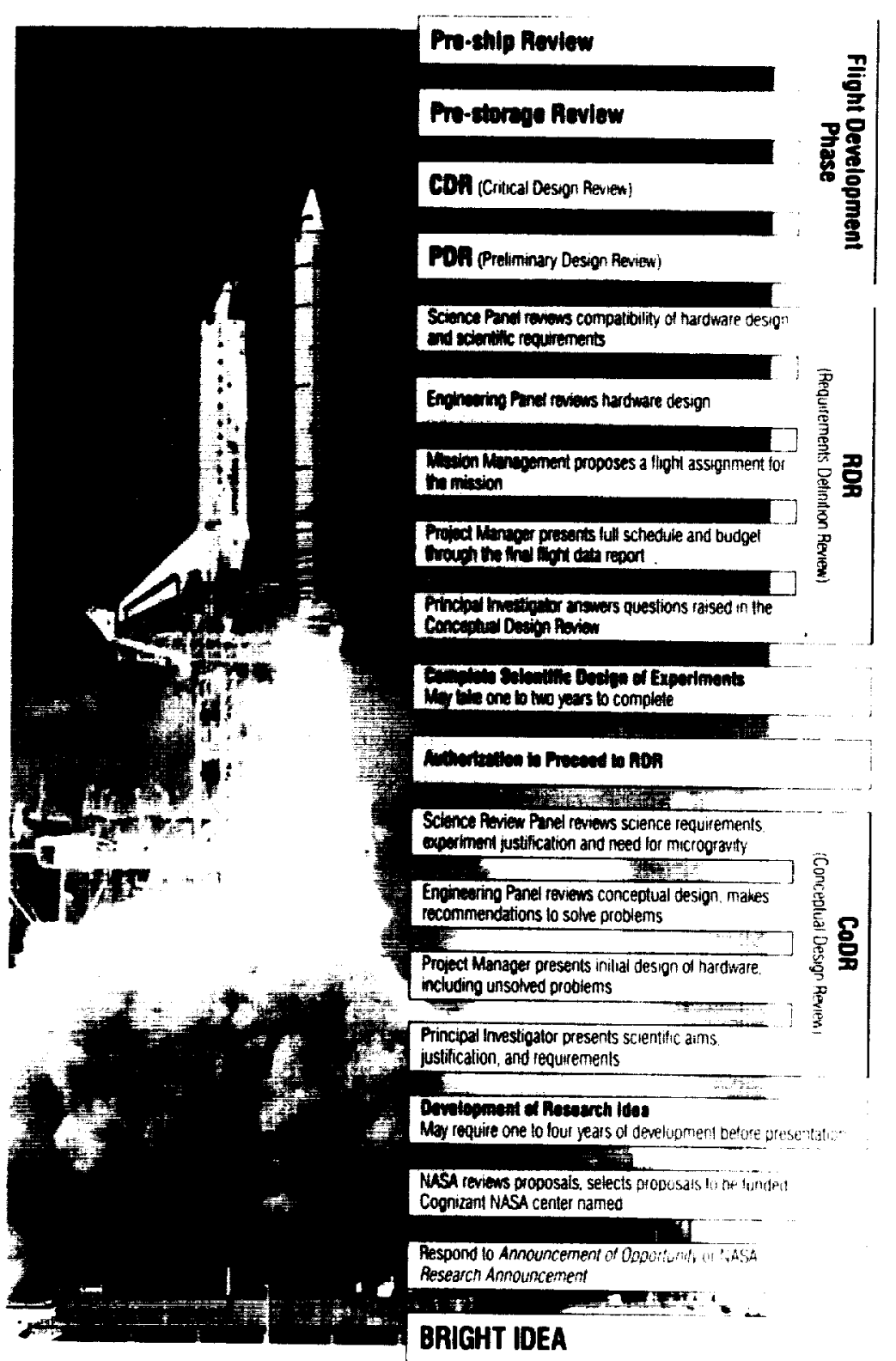

FIGURE 8.4 Elements of the flight experiment development process. 

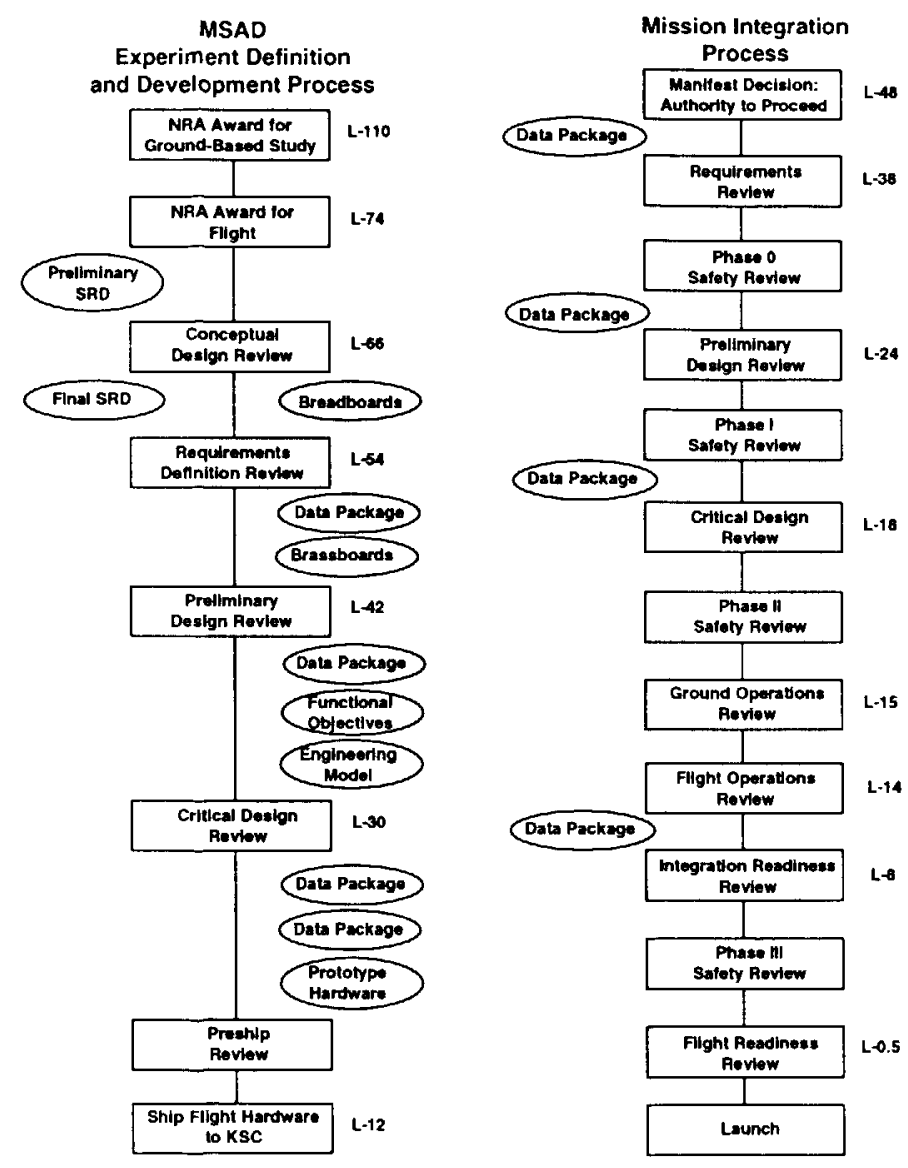

FIGURE 8.5 USML-1 space experiments processes MSAD, Microgravity Science and Applications Division; NRA, NASA Research Announcement: KSC. Kennedy Space Center; SRD, Science Requirements Document.

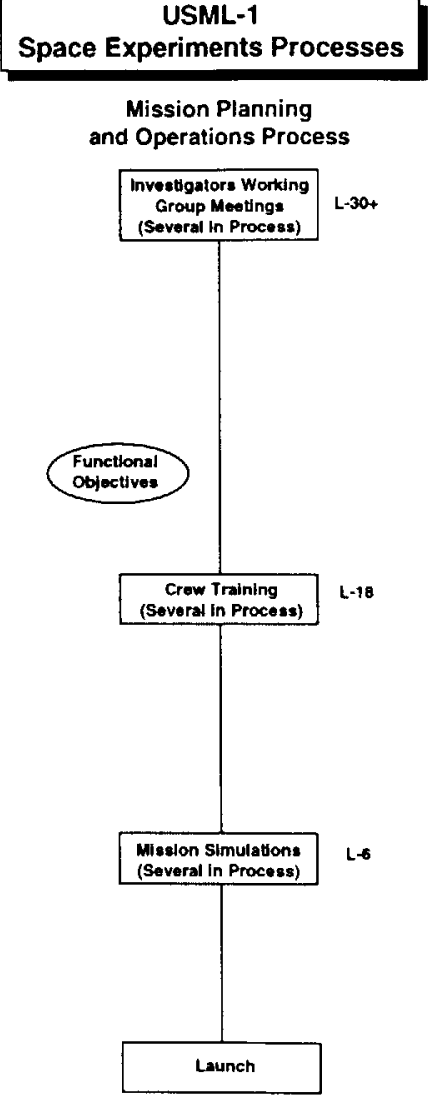


It is suggested that (1) the interactions among centers and headquarters be simplified and unified and (2) the principal investigators be continually involved in all aspects of these procedures. Every procedure and requirement should be examined to determine if it is relevant and essential. 
APPENDIXES 



\section{A \\ Glossary}

Advective: A mode of heat and species transport associated with the directed motion of a fluid as often induced by volume changes accompanying phase transformations.

Anisotropic: The variation of some material property with respect to direction or spatial orientation.

Austenite: The high-temperature phase in steel consisting of carbon dissolved in face-centered cubic iron.

Batch technique: One of several strategies for crystallization in which a supersaturated condition for one dissolved component is established by mixing solutions and other additives into a single batch solution. In the typical application, this solution is then left undisturbed during precipitation of the supersaturated component in the form of crystals.

Biochemical assays: Methods of detecting and monitoring protein or other biological activities. The activity of an enzyme, for example, may be monitored by the disappearance of reactants or the appearance of products from the reaction it catalyzes.

Biological macromolecule: One of a diversity of large molecules (relative molecular weights typically 5000 or more) characteristic of biological systems, such as proteins, nucleic acids (e.g., DNA), and polysaccharides.

Biomimetic synthesis: Synthesis by processes that mimic those found in biological systems. An example is the composite organic mineral structure of abalone shell. 
Bridgman: A technique for effecting bulk crystal growth by application of a temperature gradient to a solution of the components.

Calorimetry: A classical measurement technique based on the temperature change observed in a material arising from the admission or release of a known quantity of heat energy.

Cell fusion: The merging of more than one cell into a single entity surrounded by a composite membrane, with concomitant mixing of cell contents.

Cell matrix: A structural component organizing and shaping cells in organs and tissue. A common molecular component of mammalian matrix is collagen.

Chemotaxis: A behavioral response of certain living organisms mediated by interactions of a receptor with a specific chemical that causes movement of the organism in response to a gradient in concentration of the chemical. An example is the movement of certain bacteria toward the source of a soluble nutrient.

Chromatography: A collection of methods for separang solution components by differential flow rates through a porous medium due to differences in size, charge, or binding affinity for the medium.

Coercivity: The magnitude of the magnetic field intensity required to reverse the magnetic induction from one state of magnetization to its opposing orientation.

Commercial enzymes: Although biological materials have been used in generating products of commercial value for centuries, in recent times the sources of the utility of some of these materials have been identified as enzymes, an example of which is rennin, an enzyme used in commercial cheese production. More recently, interest has grown in the design and manufacture of enzyme products with commercial value. An example is the engineering of thermostable proteindegrading enzymes such as subtilisin as additives conferring improved stainremoving properties on laundry products.

Constitutive equations: Equations that relate electric displacement with electric field intensity and magnetic induction with magnetic field intensity.

Containerless: The description of materials processes carried out in microgravity that precludes the need for a supporting vessel or crucible and thereby eliminates sources of contamination at high temperatures.

Convective: A mode of heat and species transport in fluid systems caused by bulk (macroscopic) motion of the fluid.

Cuprate superconductors: A class of high-temperature superconductors based on layered intermetallic compounds containing copper oxide and metals such as yttrium, barium, and bismuth.

CVD: Chemical vapor deposition. 
Czochralski method: A technique for effecting bulk crystal growth in which a "seed" crystal grows by its gradual withdrawal from a stationary melt phase.

Dendritic: A branched, tree-like, form of crystal growth, commonly occurring in the solidification of metals and alloys during casting and welding.

Diffusivity: The transport coefficient that relates the net flux of species or heat flowing in response to a gradient of the concentration or temperature, respectively, established in the material.

Flectrofusion: Cell fusion facilitated by application of electric fields.

Electromagnetic levitator: A device consisting of a high-frequency coil that provides a magnetic lifting force and heating for a conducting material placed within the coil.

Electroosmosis: Transport across a semipermeable membrane driven by electrical potential difference.

Electrophoresis: Separation of charged molecules based on differential mobilities in an imposed electrostatic field.

Electrowinning: The process of recovery of metallics from low-grade ores by leaching the mineral with an acidic solution followed by electrolytic precipitation.

Ellipsometry: An optical technique used for determining the index of refraction and other optical properties of the surface of a material by analyzing the elliptically polarized light reflected from the surface.

Embryogenesis: The collection of complex developmental processes involved in going from ovum to embryo.

Emissivity: The optical property of a material body that relates the absorbed energy flux for a given wavelength to that of a perfectly absorbing substance.

Endocrine cell: A cell from one of a number of different glands that secrete molecules, known as hormones, that influence the behavior of other cells.

Epitaxy: The growth on a crystalline substrate of a crystalline layer with an ordered structure that is determined by the crystal structure of the substrate. As used in this report, the epitaxial layer has the same structure as the substrate.

Eutectic: A thermodynamic reaction in which a liquid transforms into two solid phases upon cooling.

Fermentation: A process involving enzymatically controlled breakdown of nutrients in the absence of air by living organisms such as yeast.

Flammability limit: Mixture ratio limits of fuel to oxidizer above and below which a propagating flame will not sustain itself. 
$g$-jitter (gravity-jitter): The spectral range of oscillatory accelerations arising from crew motions, machinery, rocket firings, and so on, occurring in orbiting spacecraft.

GBP: Gravity Probe-B-an experimental test of magnetogravitational effects predicted by Einstein's general theory of relativity.

Genome: The collection of genetic material that encodes all of the components, the assembly, and the development of an organism.

Glovebox: A hermetically sealed environment accessible to hand manipulation by gloves that are an integral part of the environmental enclosure.

Glycoprotein: A protein that is modified after synthesis by the attachment of oligomeric assemblages of carbohydrate. The attachment sites and the nature of the attached carbohydrate are determined by the action of enzymes.

Heterostructure: Term usually applied to semiconductors. A multilayered, single-crystal structure with the layers having different compositions, but with nearly defect-free interfaces as the result of being either lattice matched or thin enough that stress has not been relieved by the formation of defects.

Hybridoma: A fused cell combining a cell with desired properties with a cancer cell that confers reproductive immortality on the fusion product.

Hydrothermal growth: Synthesis in high-pressure aqueous systems at temperatures above $100^{\circ} \mathrm{C}$.

Hypereutectic: Describing compositions of an alloy that are above the eutectic point.

Hysteresis: The memory effect exhibited in some property by systems changing from one state to another, such as from the magnetized to the unmagnetized condition.

Immiscibility: The property of liquid alloys over certain temperatures, pressures, and compositions by which they separate into distinct phases, such as oil and water at room temperature and atmospheric pressure.

Immunoglobulins: Proteins whose function it is to recognize and bind to dangerous or foreign molecules, matter, or cells, and to flag them for destruction or elimination.

Incongruent melting: Melting with decomposition.

Intercellular communication: The process by which cells influence the behavior of other cells in a multicellular organism. Communication occurs most commonly by chemical means and involves the interactions of a bewildering variety of secreted chemicals with specific cellular receptors. 
Intermetallic: Referring to phases of nearly fixed atomic proportions, usually exhibiting limited mutual solubility, and occurring away from the ends of the phase diagram.

Isoelectronic: Having the same electronic structure.

Isostatic pressing: A process for consolidating powders by application of pressure equally from all sides.

Lambda point: For ${ }^{4} \mathrm{He}$, the temperature $(2.1780 \mathrm{~K})$, at atmospheric pressure, at which the transformation between the fluids helium I and helium II takes place. At this point the heat capacity of the system increases without limit as the transition temperature is approached from above or below.

Landau-Darrieus instability: A fluid interface instability occurring when there is a density difference across the interface and mass transport across the interface.

Lewis number: Nondimensional number consisting of the ratio of the thermal and mass diffusivities.

Liposomes: Aqueous compartments enclosed by lipid bilayer membranes. Artificial liposomes are constructed to encapsulate substances for controlled delivery in biological systems.

Liquid-liquid diffusion: A method of crystallization in which two different solutions, miscible or immiscible, are brought in contact to form an interface, with or without the benefit of a semipermeable membrane. Subsequent diffusion across the interface establishes a supersaturated condition for one of the dissolved components, typically a protein, resulting in precipitation of that component in the form of crystals.

Liquidus: The curve giving the compositions and temperatures at which a liquid and a crystalline phase may coexist in thermodynamic equilibrium.

LPE: Lambda-point experiment - a high-resolution determination of the heat capacity of liquid ${ }^{4} \mathrm{He}$ at superfluid transition ( $\lambda$-point).

Macromolecular: Referring to large molecules such as those found in biological systems, where a relative molecular weight of 2000 to 5000 daltons or greater is implied, or those studied by polymer sciences.

Macrosegregation: The redistribution of solute atoms between the solid and liquid phases during solidification, with redistribution occurring over distances that are large compared to the size scale of the solid-liquid microstructure.

Martensite: A hard, metastable phase occurring spontaneously in certain rapidly cooled (quenched) steels, consisting of iron and carbon with a tetragonal crystal structure. 
Membrane: A semipermeable molecular barrier. The most well known example, the cell membrane, surrounds the cell and separates the interior from external surroundings. The typical biological membrane is a bilayer of lipid molecules exposing polar head groups to both exterior and interior.

Mesoscopic: Referring to a scale of size characterizing the microstructure of materials that typically fall in the range $10^{-5}$ to $10^{-2} \mathrm{~cm}$ (i.e.. intermediate between microscopic and macroscopic).

Metastable: The thermodynamic condition of a phase or assembly of matter in which the minimum energy state is not accessible without first passing through a state of higher energy. Such states can persist for long periods of time.

Monoclonal antibody: A single specific immunoglobulin antibody reproduced in an immortalized cell line by artificial methods.

Monodispersions: Polymer systems that are homogeneous in molecular weight.

Monomer: A molecule consisting of a group of atoms that constitute the chain repeat of a polymer.

Monotectic: A thermodynamic reaction in which a liquid alloy, upon cooling, separates into a new liquid phase and a crystalline phase.

Nanomaterials: Generic designation of bulk solid and thin film materials prepared by special processing methods to consist of ultrafine crystallites with diameters of less than $10^{-8} \mathrm{~m}$. Such materials have a preponderant number of their atoms in interfacial locations and as a consequence display unusual properties.

Nanostructures: An arrangement of almost atomically thin, contiguous layers of semiconducting materials, usually operating as an optoelectronic device. Such devices often exhibit unusual behavior based on quantum effects that depend on the reduced dimensions of the crystalline layers.

Nucleic acid (sequence): A linear polymer of a mixture of four purine- or pyrimidine-ribose-phosphate monomers. Depending on the type of ribose, the nucleic acid is either DNA or RNA. In genetically active DNA or RNA, the sequence of purines and pyrimidines, taken in triplets, encodes the sequence of amino acids in a protein.

Oligomers: Polymers made up of two, three, or four monomer units.

Organelle: One of several kinds of distinct particulate bodies, either entirely membranous or an organized unit surrounded by a membrane, found within cells. Most organelles-the cell nucleus is an example--are associated with important cell functions, and the cells that contain them (eukaryotic cells) are reckoned to be more advanced than those without (prokaryotic cells). 
Ostwald ripening: The general thermodynamic and kinetic tendency of dispersed collections of fine particles to interact and lower their energy by exhibiting shrinkage and eventual disappearance of smaller particles and simultaneous growth and dominance of larger particles.

Peptide: Part or all of a protein. Any chemical entity including a peptide bond (i.e., a bond joining amino and carboxylate groups by elimination of a water molecule).

Peritectic: A thermodynamic reaction in which a solid, upon heating, decomposes to a liquid phase and a new crystalline phase.

pH: The negative logarithm (base 10) of the hydrogen ion concentration. In an aqueous solution, the accessible range of $\mathrm{pH}$ is from 1 to 14 by virtue of the dissociation of water. Acidic conditions have $\mathrm{pH}$ values lower than 7 ; basic, higher.

Phase partitioning: Fractionation of mixtures based on differential solubilities of components in two or more immiscible phases.

Polymerization: Chemical process in which individual monomers are converted into polymers.

Polymers: Substances made up of large molecules formed by the union of simple molecules.

Polymorphic: One of many possible crystallographic forms for a solid material of given chemical composition.

Polyphase: A mixture or microstructure containing two or more coexisting phases.

Precipitant: A compound or solution that, when added to the solution of a component that is to be precipitated, establishes a supersaturated condition for that component and causes the desired precipitation.

Protein activity: The ability of a protein to perform its function. This function may require one or more of several different kinds of activities. If the protein is an enzyme, its activity is measured by its ability to catalyze a specific biochemical reaction. If the protein is a receptor on a cell surface, its activity is measured as its ability to recognize and bind a specific ligand and to transduce that ligandbinding event as a chemical signal within the cell.

Proteins: Heterogeneous linear polymers of amino acids connected by peptide bonds in sequences specified by the genetic messages encoded in DNA. In aqueous environments, these molecules typically adopt characteristic three-dimensional folded structures that endow them with the properties required for the functions they perform. 
Pyrolysis: Gasification of a condensed-phase substance with, perhaps, chemical change by the application of heat.

Rayleigh-Taylor instability: The instability of the interface separating two fluids having different densities when the lighter fluid is accelerated toward the heavier fluid.

Receptor-ligand interactions: The collection of phenomena comprising and attending the recognition and binding of a receptor to a specific ligand molecule and the transduction of that event through the cell membrane.

Recombinant DNA techniques: The collection of techniques that are the basis for genetic engineering capabilities. Included are isolation of a specific RNA message, cloning of DNA complementary to the message, insertion of this DNA into transferable genetic elements, and infection of a foreign host cell with these genetic elements for expression of the selected gene.

Reynolds number: A dimensionless number indicating the ratio of inertial to viscous forces in a fluid flow.

Ribosome: The assembly of proteins that translates a genetic instruction in the form of an RNA message and synthesizes the encoded protein.

Shadowgraph, schlieren: Methods of making visible the disturbances in fluid flows by refracting light through density gradients in the fluids.

Sintering: The process of powder particle bonding and coalescence by diffusion.

Solutal: Referring to alloy additions and/or chemical impurities, such as solutal convection.

SQUID: Superconducting Quantum Interference Device that provides state-ofthe-art magnetic field measurements.

Stoichiometry: The whole-number relationship between the number of moles of each element constituting a chemical compound.

Stokes regime: Flows with a Reynolds number of less than 1 .

Superalloys: Any of the complex nickel-based alloys used for high-temperature service in gas turbines and jet engines.

Supercool: Referring to the persistence of a (metastable) liquid phase at temperatures below the normal melting point, where, strictly speaking, only the crystalline form is thermodynamically stable.

Thermocapillary: Referring to changes in surface tension due to temperature variations that can generate fluid motions. 
Thermodiffusion: The transport of matter induced by application of a temperature gradient.

Thermophoresis: The kinetic phenomenon in which a small particle or molecule moves spontaneously and collinearly to the applied thermal gradient.

Thermosolutal: Referring to combined convection effects arising from density gradients associated with alloy additions and temperature.

Virus: One of a complex variety of parasitic particles capable of replicating itself by infection of a host cell and consisting in its simplest form of an RNA or DNA genome surrounded by a coat of viral protein.

Viscoelastic: Referring to mechanical behavior in which both fluid-like (viscous) and solid-like (elastic) characteristics are exhibited.

Weldments: A generic term referring to joints and connections between like and unlike materials made without mechanical fasteners, usually by fusing the materials together. 


\section{B \\ Biographies of Committee Members}

William A. Sirignano, Chair (199/-1994). Professor of Mechanical and Aerospace Engineering and of Chemical Engineering and Materials Science, University of California, Irvine. Ph.D., Aerospace Engineering, Princeton University, 1964. Fellow of the American Institute of Aeronautics and Astronautics (AIAA), American Society of Mechanical Engineers (ASME), and American Association for the Advancement of Science (AAAS). Previously served on NASA Space Science and Applications Advisory Committee, AIAA Solid Rockets Technical Committee, AIAA Propellants and Combustion Technical Committee, University Space Research Association (USRA) Microgravity Science Council, and the Combustion Institute Executive Committee and Board of Directors. Research interests: spray combustion, turbulent combustion, ignition, aerospace propulsion, automotive combustion, energy conservation and pollution, fire research, noise suppression, and applied mathematics.

Rosalia N. Andrews. Professor of Materials Science and Engineering, University of Alabama at Birmingham. Ph.D., University of Florida, 1976. Coinvestigator on USML- 1 and USML-2 HgZnTe crystal growth experiments. Member, Executive Committee of the American Crystal Growth Association. Research interests: bulk growth and characterization of group II-VI semiconducting compounds.

Howard M. Einspahr. Research Fellow and Director, Macromolecular Crystallography, Bristol-Myers Squibb Company. Ph.D., Chemistry, University of Pennsylvania, 1970. Previously served on NASA Advanced Protein Crystal Growth Advisory Committee. Research interests: protein structure, drug design. 
Martin E. Glicksman, Chair (1994-1997). Professor of Materials Science, Department of Materials Engineering, Rensselaer Polytechnic Institute. Ph.D., Physical Metallurgy. Rensselaer Polytechnic Institute, 1961. Principal investigator for Isothermal Dendritic Growth Experiments on USMP-2 (March 1994). Research interests: solidification and crystal growth of metals and alloys, surface energy studies, defects and diffusion in metals, thermodynamics of stressed systems, interfacial phenomena in metals, metallurgy of superconductors.

Franklin D. Lemkey. United Technologies Research Center. Ph.D., Metal Physics, Oxford University. Fellow of the American Society for Metallurgists (ASM). Chair, Editorial Review Board of Metallurgical Transactions. Previously, Director of NASA Microgravity Science and Applications Division (1989) and Chair of NASA's Space Station Science and Applications Users Committee (1988). Currently serves on the National Research Council (NRC) Space Station Committee of the Aeronautics and Space Engineering Board. Research interests: collaborative research and development activities with the new independent states of the former Soviet Union.

Ronald E. Loehman. Manager, Advanced Materials Laboratory, Sandia National Laboratories. Ph.D., Solid State Chemistry, Purdue University, 1969. Fellow, American Ceramic Society. Associate editor of the Journal of the American Ceramic Society. Research interests: glass-metal and ceramic-metal reactions and interface bonding, preparation and properties of nitrogen ceramics and glasses, electrical and thermal behavior of ceramics and glasses.

Alexander McPherson. Professor of Biochemistry, University of California, Riverside. Ph.D., Biological Sciences, Purdue University, 1970. Principal investigator for protein crystal growth (PCG) experiments on IML-1, IML-2, U.S.Russian Mir-Shuttle Mission. Research interests: analysis and determination of the atomic structures of biological macromolecules by $\mathrm{x}$-ray diffraction techniques and their correlation with mechanistic properties.

Simon Ostrach. Professor of Engineering, Case Western Reserve University. Ph.D., Applied Mathematics, Brown University, 1950. Member, National Academy of Engineering (NAE; currently Home Secretary); Fellow of the American Academy of Mechanics, AIAA; Honorary Member of the ASME. Research interests: fluid mechanics, natural convection, transport phenomena in industrial processes, physiochemical fluid dynamics.

Morton B. Panish. AT\&T Bell Laboratories (retired). Ph.D., Physical Chemistry, 1954. Previously. Head of the AT\&T Bell Laboratories Material Science Research Department. Member, NAE and National Academy of Sciences (NAS). Fellow of the American Physical Society and the Institute of Electrical and Electronics Engineers (IEEE). Research interests: group III-V compound phase chemistry, impurity incorporation. epitaxy, device structures. 
John D. Reppy. Professor of Physics, Cornell University. Ph.D., Physics, Yale University, 1961. Member NAS; Fellow of the American Physical Society, American Academy of Arts and Sciences, New York Academy of Sciences, and AAAS. Research interests: low-temperature physics, quantum properties of superconductors and superfluid helium, cooperative and critical phenomena.

Warren C. Strahle (deceased). Regents Professor, School of Aerospace Engineering, Georgia Institute of Technology. Ph.D., Aerospace Engineering, Princeton University, 1964. Fellow AIAA. Previously served on the NASA Microgravity Combustion Discipline Working Group; AIAA Aeroacoustics Technical Committee, Solid Rockets Committee, Propellants and Combustion Committee. Associate editor of the AIAA Journal. Research interests: liquid and solid rocket combustion stability, thrust vector control, external and supersonic combustion, tactical and strategic missile preliminary design, ramjet combustion stability, combustion noise, diesel engine noise, underwater explosions, and turbulent reacting flows.

Julia R. Weertman. Professor, Materials Science and Engineering, Northwestern University. Ph.D., Carnegie Institute of Technology (now Carnegie Mellon University), 1951. Member NAE; Fellow of the ASM International and of the Minerals, Metals, and Materials Society. Research interests: mechanical behavior of metals and alloys, especially nanostructured materials, structural characterization. 